\title{
CLINICAL ACCEPTABILITY OF ORTHODONTIC BRACKET BASE-CEMENT COMBINATIONS
}

by

\section{Zahra Hassanloo}

A thesis submitted in conformity with the requirements

for the degree of Masters of Science

Graduate Department of Orthodontics

University of Toronto

@ Copyright by Zahra Hassanloo (2001) 
National Library

of Canada

Acquisitions and Bibliographic Services

395 Wellington Street Ottawa ON K1A ONA Canada
Bibliothèque nationale du Canada

Acquisitions et services bibliographiques

395. rue Wellington

Ottawa ON K1A ONA

Canada
The author has granted a nonexclusive licence allowing the National Library of Canada to reproduce, loan, distribute or sell copies of this thesis in microform, paper or electronic formats.

The author retains ownership of the copyright in this thesis. Neither the thesis nor substantial extracts from it may be printed or otherwise reproduced without the author's permission.
L'auteur a accordé une licence non exclusive permettant à la Bibliothèque nationale du Canada de reproduire, prêter, distribuer ou vendre des copies de cette thèse sous la forme de microfiche/film, de reproduction sur papier ou sur format électronique.

L'auteur conserve la propriété du droit d'auteur qui protège cette thèse. $\mathrm{Ni}$ la thèse ni des extraits substantiels de celle-ci ne doivent être imprimés ou autrement reproduits sans son autorisation. 


\section{ABSTRACT \\ CLINICAL ACCEPTABILITY OF ORTHODONTIC BRACKET BASE-CEMENT \\ COMBINATIONS Z. HASSANLOO, Faculty of Dentistry, Graduate Department of Orthodontics, University of Toronto, Canada. 2001}

The aim was to identify bracket base-cement combinations that are clinically practical, provide adequate shear bond strengths (SBS), and result in the least damage to enamel. The SBS and the nature of bond failure using the Adhesive Remnant Index (ARI) were examined after 1 and 180-days for 28 combinations. SBS in $8 \pm 3 \mathrm{MPa}$ range and ARI scores $\geq 2$ were deemed clinically acceptable. Transbond and Fuji Ortho LC (FOL) (conditioned) were compatible with the four brackets ( 2 metal: Time, Speed ; and 2 ceramic: Clarity, Transcend). Panavia21 was compatible with ceramic brackets. FOL (not-conditioned) yielded poor SBS and ARI scores with all of the bracket bases. Time had no significant effect on clinical acceptability. Enamel conditioning had a significant effect on the ARI scores $(p<0.05)$ and SBS $(p<0.05)$. No consistent association was noted between the ARI scores and SBS. The ideal cement for bonding the four brackets was FOL (conditioned). 


\section{ACKNOWLEDGMENTS}

I would like to express my appreciation and sincere gratitude to the following individuals for their invaluable assistance with the successful completion of this thesis:

Dr. K.C. Titley, my thesis supervisor, for his meticulous attention to detail, guidance and assistance with all the aspects of this project.

Dr. G.V. Kulkarni, for his statistical analysis, expertise and advice in preparation of this thesis.

Dr. P.E. Rossouw, for his advice and participation in my thesis advisory committee.

Mr. Robert Chernecky, for his technical assistance with the Scanning Electron Microscope and for his kind friendship.

Dr. J.T. Mayhall, for the generous use of the Morphometric Program and for his help with the digitizing of the bracket bases.

Thank you to my family for their unconditional love and support. Lastly I like to dedicate this thesis to my mother, Azam, for having made everything good in my life possible. 


\section{TABLE OF CONTENTS}

Abstract ii

List of Tables vii

List of Figures ix

Introduction and Statement of the Problem 1

Review of the Literature 4

Metal Brackets

Perforated Bases 4

Foil-mesh Bases $\quad 5$

Integral Bases $\quad 5$

Sintered Bases $\quad 6$

Photo-etched Bases 6

Sandblasting (microetching) $\quad 7$

$\begin{array}{ll}\text { Polycarbonate Brackets } & 7\end{array}$

Ceramic Brackets

Polycrystalline Brackets $\quad 8$

Monocrystalline Brackets 9

Bracket Retention Mechanisms and Base Surface Characteristics 9

Ceramic Bracket Bond Strength and Fracture Mode 10

Debonding of Ceramic Brackets $\quad 11$

\section{Bonding Cements}

Resin Cements 12

Acrylic and Diacrylic Resin Cements 12

Filler Content $\quad 13$

Auto-polymerized and Photo-polymerized Resin Cements 14

Dual Cured RCLCs 17

$\begin{array}{ll}\text { Glass Ionomer Cements } & 17\end{array}$

Resin-Reinforced Glass Ionomer Cements 19

Conditioned and Non-conditioned Enamel Surfaces 21 


\section{Types of Enamel Surface Conditioners}

Acid Conditioning 23

Crystal Growth $\quad 25$

Adhesive Primer $\quad 27$

Storage Time $\quad 29$

Human and Bovine Enamel 30

Bond strength Testing 32

Location of Bond Failure $\quad 33$

Objectives of the Study

Operational Definitions 36

Materials and Methods

Bracket Base Types and Cements 38

Bracket Base Bonding Surface Area

Preparation of the Teeth 40

Bonding Protocol 442

Storage 43

Shear Bond Strength Testing

Nature of Bond Failure $\quad 44$

Inter and Intra-Operator Error Study on ARI

Scanning Electron Microscopy 44

Statistical Analysis $\quad 45$

\section{Results}

1.Clinical acceptability of various bracket base-cement combinations

i. 1-day storage group 46

ii. 1-180 days storage group 46

\section{Comparison of mean SBS (1-day of storage)}

i. Comparison of mean SBS of various bracket base-cement combinations $\mathbf{5 2}$

ii. Comparison of mean SBS of various bracket bases 52

iii. Comparison of mean SBS of various cements 53

3. Comparison of mean SBS (180-days of storage)

i. Comparison of mean SBS of various bracket base-cement combinations 54

ii. Comparison of mean SBS of various cements 56

4. The effects of long-term storage on mean SBS of various bracket base-cement combinations

i. Speed brackets bonded with different cements 59

ii. Time brackets bonded with different cements 60

iii. Transcend brackets bonded with different cements 61

iv. Clarity brackets bonded with different cements 62 
Comparison of ARI scores associated with various cements

6. The mean ARI scores for various bracket base-cement combinations (1-day of storage)

i. The mean ARI scores for brackets bonded with Transbond

ii. The mean ARI scores for brackets bonded with Panavia21

66

iii. The mean ARI scores for brackets bonded with Fuji Ortho LC 66 (conditioned)

iv. The mean ARI scores for brackets bonded with Fuji Ortho LC 66 (not-conditioned)

7. ARI scores distribution (180-days of storage)

Comparison of ARI scores associated with various cements

8. The mean ARI scores for various bracket base-cement combinations (180-days of storage)

i. The mean ARI scores for brackets bonded with Transbond

68

ii. The mean ARI scores for brackets bonded with Panavia21

69

iii. The mean ARI scores for brackets bonded with Fuji Ortho LC (conditioned)

9. The effects of long-term storage on the frequency of ARI scores distribution

10.The relationship between SBS and ARI scores distribution for various bracket base-cement combinations ( 1 and 180-days of storage)

11. Effects of conditioning the enamel with $10 \%$ polyacrylic acid on the mean ARI Scores of brackets bonded with Fuji Ortho LC cement

12. Compatibility between bracket base design and the cement particle size

13. Error Study

Discussion

Future Research

Conclusions

Bibliography 


\section{LIST OF TABLES}

Table 1. ARI Scoring Index $\quad 34$

$\begin{array}{lll}\text { Table 2. Bracket Types and Their Bases } & 38\end{array}$

Table 3. Properties and Characteristics of the Bonding Cements 39

Table 4. Bracket Bonding Base Area 40

Table 5. Experimental Protocols 41

Table 6: Shear Bond Strength (SBS) and Adhesive Remnant Index (ARI) 50

Table 7. Shear Bond Strength (SBS) and Adhesive Remnant Index (ARI) 51

Scores Percent Distribution (180-days of storage)

Table 8. Two-way Analysis of Variance for Mean SBS of 52

4 Brackets and 4 Cements/Bonding Protocols Tested (1-day of storage)

Table 9. Duncan's Grouping of the Mean SBS for Different Bracket Base Types 53

Table 10. Duncan's Grouping of the Mean SBS for Different Cement Materials 54 (1-day of storage)

Table 11. Two-way Analysis of Variance for SBS of 4 Brackets and $4 \quad 55$ Cements/Bonding Protocols Tested (180-days storage)

Table 12. Duncan's Grouping of the Mean SBS for Different Bracket Base Types (180 days- of storage)

Table 13. Duncan's Grouping of the Mean SBS of Various Cements (180-days of storage)

Table 14. The Pair wise Comparison of Mean SBS of Various Bracket BaseCement Combinations (1-day storage)

Table 15. The Pair wise Comparison of Mean SBS of Various Bracket BaseCement Combinations (180-days storage)

Table 16. Comparison of the Mean Shear Bond Strength (SBS) ( 1 and 180-days of storage)

Table 17. Mean ARI Scores for Various Bracket Base-Cement Combinations 1-day of storage) 
Table 18. Duncan's Grouping of the ARI Scores Associated with

Table 19. Mean ARI Scores for Various Bracket Base-Cement Combinations (180-days of storage)

Table 20. Duncan's Grouping of the ARI Scores Associated with

Different Cement Materials (1-day of storage)

Table 21. Effects of Storage Time on ARI Scores

Table 22. Mean SBS and Corresponding ARI Scores Distribution for Speed Bracket Bonded with Different Cements (1 and 180-days of storage)

Table 23. Mean SBS and Corresponding ARI Scores Distribution for Time

Bracket Bonded with Different Cements (1 and 180-days of storage)

Table 24. Mean SBS and Corresponding ARI Scores Distribution for Transcend Bracket Bonded with Different Cements (1 and 180-days of storage)

Table 25. Mean SBS and Corresponding ARI Scores Distribution for Clarity Bracket Bonded with Different Cements (1 and 180-days of storage)

Table 26. Comparison of Mean Shear Bond Strengths of Brackets Bonded with Fuji Ortho LC with and without Enamel Conditioning

Table 27. Comparison of ARI Score Distribution and the Mean ARI Scores of 82 Brackets Bonded With Fuji Ortho LC

Table 28. Compatibility between Bracket Base Design and the Cement Particle Size 83

Table 29. ARI Scores Assigned by Two Different Investigators $\quad 85$

Table 30. ARI Scores Assigned by the Principal Investigator at Two Different 86 Times 


\section{LIST OF FIGURES}

Figure 1.

Figure 2.

Figure 3.

Figure 4.

Figure 5.

Figure 6.

Figure 7.
Mean ARI Scores of Various Bracket Base-Cement Combinations Tested after 1 and 180-Days of Storage

for Speed Brackets Bonded with Different Cements

Comparison of Mean SBS after 1 and 180-Days of Storage for Time Brackets Bonded with Different Cements

61

Comparison of Mean SBS after 1 and 180-Days of Storage for Transcend Brackets Bonded with Different Cements

Comparison of Mean SBS after 1 and 180-Days of Storage 62 for Clarity Brackets Bonded With Different Cements

Compatibility of Different Bracket Base Designs and Particle 84

Size of Various Cements 


\section{INTRODUCTION AND STATEMENT OF THE PROBLEM}

The conditioning of the enamel surface with phosphoric acid (Buonocore, 1955) and the development of composite resin cements (Bowen, 1962) revolutionized aesthetic dentistry and the practice of orthodontics. Today, the direct bonding of brackets to enamel has become routine in fixed orthodontic appliance therapy. Clinicians have an extensive selection of bracket bases and bonding cements at their disposal so that their choices can be tailored to the patients' specific needs, their own clinical experience, product monographs and published research pertaining to the properties and endurance of these materials. Despite the numerous publications in the field of orthodontic bonding, the orthodontic literature lacks studies that identify the most suitable bracket base-cement combinations. Similarly, there is no information on the interaction between bracket base design and cement particle size. Furthermore, bracket base-cement bond failure still occurs and this is costly to both patients and clinicians and may prolong treatment time.

Given the wide variety of orthodontic bracket base designs and the myriad of bonding cements available today, it is important to identify bracket base-cement combinations that are:

- Clinically practical

- Provide adequate shear bond strengths

- Result in minimal or no damage to enamel during bonding, orthodontic treatment and debonding

Information on the interaction between bracket base design and cement particle size will add to orthodontic knowledge and help manufacturers to develop compatible systems.

Important variables, which affect the bond strength of bracket bases to enamel, are cement type, bracket material and base design, enamel surface treatment and storage time.

The choice of brackets is dependant on the clinician's preference and the aesthetic requirements of the patient. There are currently three bracket materials available, and they are: metal, plastic and ceramic. Each of these bracket types employs different mechanisms of retention, that are incorporated into their base designs. In metal and plastic brackets, the 
bond strength is primarily derived from mechanical mechanisms, which include the welding of a mesh pattern to the base (metal brackets) or the incorporation of micro-porosities (plastic brackets). However, ceramic brackets may rely on both mechanical and or chemical mechanisms for bond strength enhancement. Consequently, during debonding of ceramic brackets, enamel may be damaged because of increased bond strengths. It is thus of clinical significance to identify cement types for ceramic bracket bases, which result in lower yet clinically acceptable bond strengths and favourable bond failure locations.

Resin containing luting cements (RCLCs) and the resin reinforced glass ionomer cements (RRGICs) are the two major adhesive systems, which are used in direct bonding of orthodontic attachments. RRGICs have received special attention as bonding agents in orthodontics, because of their ability to release fluoride with its beneficial cariostatic effect. An added benefit is the claim that RRGICs do not require any acid conditioning so that it may be concluded that enamel loss due to acid conditioning and subsequent decalcification can be prevented. RRGICs can also be bonded on to a moist enamel surface. The current view on the use of enamel conditioner with RRGICs is equivocal some investigators have reported success with RRGICs even when enamel surface is not conditioned (Fricker, 1994 and Silverman et al., 1995). However, more studies are concluding that RRGICs do not provide adequate bond strength without the use of an enamel conditioner (Beress et al., 1998; Bishara et al., 1998b; Flores et al., 1999; Lippitz et al., 1998; Meehan et al., 1999; Millett et al., 1999). It is of clinical significance that RCLCs and RRGICs, with and without enamel conditioning, are tested to evaluate and compare their shear bond strengths (SBS) when used to bond different types of bracket bases.

New products are formulated with the object of both saving time and providing cost effective materials. The self-etching acidic primer, a combination of an acid and a primer, is advertised as a product, which combines both acid conditioning and primer application into one step. Acid conditioning and unfilled resin primer (sealant) application are the steps required in bonding bracket bases when using resin cements. A study by Bishara et al. (1998a) showed that the use of acidic primers to bond orthodontic brackets to the enamel surface provided clinically acceptable SBS when used with a highly filled RCLC 
(Panavia21). However, more research is needed to test the compatibility of this bonding system (Acidic primer + Panavia21) using metal, and ceramic brackets.

The aim of this study was to identify combinations of orthodontic bracket bases and bonding cements that can provide clinically acceptable SBS at 1 and 180-days following bonding while resulting in minimal or no damage to the enamel. The effects of bracket base material and design, cement type, enamel surface treatment and storage time on the shear bond strength and bond failure pattern were assessed. Furthermore, the compatibility of four bracket base designs with three different cement particle sizes was determined. 


\section{REVIEW OF THE LITERATURE}

Orthodontic bracket bond failure and the need for rebonding is a time consuming process which results in frustration for both the orthodontist and the patient, additional costs in materials and personnel, increased treatment time and possible damage to tooth structure. The variables affecting the bracket base bond strength are: 1) the bracket material and more particularly the base design, 2) the type of cement, 3) the mode of enamel surface treatment prior to bonding, and 4) storage time. The following is a brief literature review of these variables and the results of the pertinent studies. This review will also explore the types of bond failure and discuss the suitability of bovine enamel as a substitute for human enamel in bonding studies.

\section{BRACKET MATERIAL}

The choice of brackets is dependent upon the clinician's preference and the aesthetic requirements of the patient. There are three bracket materials available: metal, polycarbonate, and ceramic.

\section{Metal Brackets}

Metal brackets, which are composed of stainless steel, were the first brackets to be introduced following the advent of enamel bonding. The major drawback of metal brackets is their associated poor aesthetics. In metal brackets the bond strength to the bracket base and tooth surface is primarily achieved by mechanical mechanisms. Although resin type cements bond well to a conditioned dry enamel surface, they do not adhere to stainless steel. Therefore, retention of these resin cements to metal bracket bases is primarily mechanical so that any bracket base design must incorporate a mechanical locking device for the cement to permeate prior to polymerisation. Following is a brief review of the different base designs that impart mechanical retentive properties to metal bracket bases.

\section{Perforated Bases}

When first introduced metal brackets had perforated bases in order to mechanically attach to cements (Thanos et al., 1979). When bonding these brackets the cement was free to flow through the holes, that were not obliterated by the bracket. The major problem associated 
with this type of base was plaque retention and poor aesthetics. Brackets with perforated bases are no longer used in orthodontics.

\section{Foil-mesh Bases}

Foil-mesh bases replaced perforated bases. The foil-mesh is either welded or brazed on to the bracket base. Compared to a perforated base foil mesh provides a smoother, less plaque retentive and thus more hygienic surface (Maijer and Smith, 1981). It has also been shown that foil-mesh bases provide better retention than perforated metal bases (Reynolds and von Fraunhofer, 1976; Faust et al., 1978; Thanos et al., 1979; Lopez, 1980). Weld spots used to secure the foil mesh to the bracket are thought to reduce the retentive area and as a result reduce the bond strength by obliterating distinct areas of the foil-mesh (Dickinson and Powers, 1980). Maijer and Smith (1981) also suggest that these weld spots act as "stress concentrators" in composite resin, thus leading to a weakened bond between the base and the cement. However, using a laser for welding or mesh attachment by brazing yields better tensile and shear bond strengths (Dickinson and Powers, 1980; Lopez, 1980; Maijer and Smith, 1981).

The mesh size (gauge) which refers to the number of openings per linear inch (Thanos et al., 1979) is reported to affect bond strength (Thanos et al., 1979). Studies by Maijer and Smith (1981) have reported that a 60 to 70-gauge mesh bracket base in combination with lightly filled resin cement gives the best results. These observations are similar to those made by Thanos et al. (1979) who achieved the best bond strengths using 60-gauge mesh bracket bases. Reynolds and Von Fraunhofer (1976) specifically related their work to mesh size and bond strength and demonstrated that maximum bond strength occurred when mesh sizes of 50 to 70 -gauge were used.

\section{Integral Bases}

Metal brackets with integral bases are fabricated in a manner that both the bracket and its base are cast as an integral unit. As a result, the bracket cannot be separated from its base (Mahal, 2000). The integral base brackets are divided to two types: machined integral brackets and cast integral brackets. In machined integral brackets a milling process is used 
for imparting mechanical retention on to the bracket base, whereas for cast integral base brackets a casting process is used. The bases of integral brackets have horizontal undercut channels, which open at the mesial and distal extremities (Ferguson et al., 1984). The Time bracket (American Orthodontics) is an example of a machined integral, miroetched base with mechanical undercuts. Sharma (1999) compared the shear bond strengths of different metal brackets and reported that the highest 24-hours bond strengths were achieved with the Time brackets (machined, integral, microetched base with mechanical undercuts) followed by Speed brackets (60-gauge, microetched foil-mesh base). When compared to foil-mesh bases, the cast integral bases show higher bond strengths, but the machined integral bases show lower bond strengths (Deidrich and Dickmeiss, 1983; Regan and Van Noort, 1989). The latter authors attribute this observation to the fact that the cast base has a much rougher surface thus increasing overall retention due to greater micro-mechanical retention.

\section{Sintered Bases}

Sintering is a process that is used to improve the mechanical retention of the cement on metal base brackets and it involves fusion of metal or ceramic particles onto the bracket base. This process creates a porous layer with increased surface area into which the cement material can penetrate. These bases are shown to yield higher tensile bond strengths than conventional foil-mesh bases (Hanson et al., 1983; Smith and Majier, 1983).

\section{Photo-etched Bases}

An alternative to foil-mesh metal bracket bases is photo-etching. Photo-etched bracket bases are retained through small indentations in the base, that are microscopically roughened by an etching process (Lopez, 1980; Maijer and Smith, 1981). The bond strength achieved by using this type of base design is greater than perforated bracket bases but less than the foilmesh bases (Lopez, 1980; Ferguson et al., 1984). The inferior bond strength compared to foil-mesh bases is attributed to poor penetration of resin cements which results in air inclusions that inhibit polymerization of uncured resin (Maijer and Smith, 1981). 


\section{Sandblasting (microetching)}

Sandblasting uses a high-speed stream of aluminum oxide particles propelled by compressed air (Black 1950, Goldstein 1994). Sandblasting of bracket bases leads to micro-roughness and subsequent increases in the surface area to enhance bond strength (Willems et al., 1997). Dietrich and Dickmeiss (1983) reported that sandblasting increases bond strengths by $34 \%$ when compared to untreated brackets. MacColl (1995) found that sandblasting of foil-mesh bases of brackets with four different base sizes significantly increased bond strengths as compared to untreated foil-mesh base. Simoka and Powers (1985) reported a 56\% increase in bond strengths when sandblasting integral grooved bases. In this study sandblasting foilmesh bases was shown to have no effect on bond strength. In a study by Millet et al. (1993), 60-micon alumina was used to sandblast foil-mesh bases that were to be bonded with glass ionomer cements. Their results showed that sandblasting for 3 seconds significantly increased bond strength whereas sandblasting for 9 seconds damaged and distorted the wire mesh. Newman et al. (1995), using auto-polymerizing resin containing luting cements, showed that sandblasting increased bond strengths by almost $20 \%$.

\section{POLYCARBONATE BRACKETS}

The current trend in orthodontics includes an ever-increasing interest of adult patients in orthodontic therapy and their insistence on the use of aesthetic appliances. Attempts to develop inconspicuous attachments lead to the introduction of polycarbonate brackets in the early 1970s. These brackets had limited success due to their water absorption and plastic deformation under load. They also stained very easily which resulted in the elimination of any aesthetic advantage (Britton et al., 1992; Reynolds, 1975). To compensate for the lack of rigidity and strength, these brackets were reinforced with metal bracket slots and ceramic fillers (Britton et al., 1992). However, the problem of slot distortion and staining remained thus restricting their clinical use. To address these problems ceramic brackets were introduced in 1986 and they have been gaining popularity ever since. 


\section{CERAMIC BRACKETS}

Ceramic is the third hardest material known to humans (Swartz, 1988a). Ceramic brackets resist staining, discoloration, and are chemically inert to oral fluids, but they are susceptible to fracture due to brittleness and low fracture toughness. The fracture toughness of ceramics (the total energy loading required to cause failure) is 20-40 times less than that of stainless steel (Phillips, 1988; Viazis, 1990). The brittleness of ceramics can result in their fracture during debonding and this will prolong the time for cleanup and increase possibility of damage to the enamel. Ceramic brackets can also cause nicks in the arch wires, resulting in more friction between the bracket and the arch wire. The increased friction can decrease the efficiency of tooth movement (Pratten et al., 1990; Bishara et al., 1993). In addition, when using ceramic brackets care should be taken to prevent contact of the ceramic bracket with opposing enamel surfaces to prevent wearing of enamel as it contacts ceramic.

Ceramic brackets are composed of either aluminum oxide or partially stabilized zirconium. The composition of most currently available ceramics is aluminum oxide. Based on the manufacturing process ceramic brackets can be divided into two basic types, namely: Polycrystalline and Monocrystalline (Swartz, 1988a).

\section{Polycrystalline Brackets}

The polycrystalline ceramic brackets are fabricated by sintering aluminum oxide particles. The process is initiated by blending the particles with a binder. The resulting mixture is then molded into a shape from which the critical parts of the brackets can be cut. The molded part is then fired at $1800^{\circ} \mathrm{C}$ to burn out the binder and fuse, but not melt the aluminum oxide particles (Swartz, 1988a). This firing process is called sintering (Bishara and Fehr, 1997). The process of molding and sintering is a popular ceramic bracket manufacturing technique owing to its relative inexpensiveness. However, this process results in not only structural imperfections at the grain boundaries but also the incorporation of trace amounts of impurities. These imperfections and impurities can serve as foci for crack propagation under stress and thus lead to fracturing of the bracket. Moreover, the grain boundaries and impurities associated with polycrystalline ceramic brackets also reflect light, which results in some degree of opacity and reduces the aesthetic effect (Swartz, 1988a). 


\section{Monocrystalline Brackets}

Monocrystalline ceramic brackets are manufactured by melting aluminum oxide particles at temperatures above $2100^{\circ} \mathrm{C}$ and allowing them to cool slowly thus permitting complete crystallization. The process minimizes the impurities and imperfections found in the polycrystalline brackets, with the result that the probability for crack propagation under stress is reduced (Bishara and Fehr, 1997). A ceramic bracket is then milled from a single crystal of aluminum oxide. This technique of manufacturing is more difficult and costly because of the hardness of the material. The process of milling and the presence of sharp corners will introduce stresses on the material and predispose ceramic brackets to fracture (Swartz, 1988a; Bordeaux et al., 1994). Milled crystal ceramic brackets are, therefore heat treated to eliminate stress inducing surface imperfections and material impurities (Swartz, 1988a). Monocrystalline brackets are essentially clear as a result of two factors: reduction in grain boundaries and fewer impurities introduced during the manufacturing process (Swartz, 1988a).

\section{Bracket Retention Mechanisms and Base Surface Characteristics}

Ceramic brackets can adhere to the luting cement through three different mechanisms:

(1) chemical, (2) mechanical, and (3) combination of mechanical and chemical (Swartz, 1988b, Karamouzos et al., 1997). Aluminum oxide, the main constituent of ceramic brackets, is an inert material, so that it cannot chemically adhere directly to any of the currently available resin containing luting cements (RCLCs) (Bishara and Fehr, 1997). As a result both mechanical and chemical modes of retention have been developed to aid in the bonding of ceramic brackets to RCLCs. Mechanical retention is achieved by producing indentations or recesses in the bracket base and these provide a mechanical interlocking with the luting cement. A chemical bond is achieved by introducing an intermediate layer of glass on the bracket base with a silane coupler thus producing a chemical bond between the bracket and the resin of the luting cement.

Ceramic bracket bases are available in four different designs (Karamouzos et al., 1997). The first design is a flat base covered with a silane layer for chemical bonding of the cement and undercuts or grooves for mechanical interlocking of the cement (Viazis et al., 1990). The 
second design includes a smooth base surface coated with a silane layer for chemical bonding with the cement (Viazis et al., 1990). The third ceramic bracket base design has a rough polycrystalline alumina base comprised of either randomly oriented sharp crystals or spherical glass particles (Eliades et al., 1994). This type of bracket base provides only micro-mechanical interlocking with the bracket bonding cement. Transcend ${ }^{2}$ and Clarity $^{1}$ brackets (3M/Unitek, CA) are both examples of this type and were tested in this investigation. The fourth design involves placement of a plastic (polycarbonate) wafer between the adhesive and the bracket base in order to facilitate debonding and avoid causing potential damage to enamel (Bordeaux et al., 1994).

\section{Ceramic Bracket Bond Strenth and Fracture Mode}

The silane coupling agent enhances the bond of the luting cement to the ceramic bracket base to such a degree that it can approach the cohesive strength of enamel with the result that enamel cracks or fracture can occur during bracket debonding (Odegaard, 1989; Carter, 1989; Storm, 1990; Machen, 1990). A number of studies have demonstrated that ceramic bracket bases which have been treated with silane coupler produce significantly stronger bond strengths when compared to conventional metal brackets (Iwamoto et al., 1987; Odegaard and Segner, 1988; Gwinnett, 1988; Ripley, 1988; Hyer, 1989; Joseph and Rossouw, 1990). An increase in the strength of the bond between ceramic bracket bases and the cement, results in bond failure at the enamel-cement interface. Bond failure at the enamel-cement interface, however, results in an increased incidence of enamel fractures and this is undesirable (Swartz, 1988b; AAO 1989; Harris et al., 1990; Joseph and Rossouw, 1990; Storm, 1990; Viazis et al., 1990). The high bond strengths between the cement and the silane coated ceramic bracket bases are of particular concern when ceramic brackets are cemented to and subsequently debonded from heavily restored teeth or non-vital teeth with brittle enamel. To reduce iatrogenic enamel fracture during debonding, some alternatives have been proposed. Guess et al. (1988) suggested that the mechanical interlocking in the bracket base provides adequate bond strength so that the additional chemical bond provided by silane treatment is not necessary. Bishara et al. (1993) showed that shear loading of mechanically retained ceramic brackets results in a cohesive failure of the cement which results in materials being left on both the enamel and bracket bases with less risk of enamel 
fracture. A further alternative to reduce bond strength between enamel and a ceramic bracket is the placement of a plastic wafer between the adhesive and the bracket base. In this situation the bond between the bracket and the wafer is the weakest so that on debonding, the bracket comes away leaving the plastic wafer and the bonding cement on the tooth surface (Fox et al., 1992; Franklin et al., 1993; Bordeaux et al., 1994).

In general, when comparing the types of cements used, the highly filled RCLCs provide a bond strength which is higher than that recorded with the lightly filled cements (Wood, 1982; Bishara et al., 1993). In the case of ceramic brackets, however, the unfilled RCLCs are reported to give comparable bond strengths to the highly filled RCLCs (Bishara et al., 1993). Alteration of enamel conditioning time was investigate by Olsen et al. (1994) as a means of subsequently reducing the force necessary to debond ceramic brackets. They showed that altering the conditioning time between 10 and 30 seconds had little influence on the bond strength or the ARI score. When the conditioning time was reduced to 5 seconds or less, the bond strength was too low to be considered clinically useful. Bishara et al. (1993) and Cacciafesta et al. (1998) also demonstrated that the use of polyacrylic acid as an enamel conditioner when cementing ceramic brackets resulted in location of bond failure that was mostly at the enamel-cement interface. According to Bishara and Fehr 1997, the use of polyacrylic acid as the enamel conditioner producus crystal growth so that neither the type of the cement nor the bracket base is critical, because the bond failure occurs within the crystals themselves. The result is that the stresses on enamel during debonding are minimized.

\section{Debonding of Ceramic Brackets}

During debonding stainless steel brackets can be "peeled" from the cement as a result of the stainless steel's ability to elongate approximately $20 \%$ before failing. Ceramic bracket materials elongate less than $1 \%$ before fracturing and, therefore cannot be "peeled" off the cement (Merril et al., 1994). Ceramic brackets can fracture during debonding because ceramic is a brittle material. To address the problems associated with debonding ceramic brackets following completion of orthodontic treatment several mechanisms of removal and a number of modifications in the bracket base design (as mentioned earlier) have been 
suggested in order to make debonding safer for the patients and less stressful and time consuming for the clinicians. Some manufacturers have developed especially designed pliers for each type of bracket, whereas some clinicians and researchers advocate their removal by electrothermal and ultrasonic methods (Bishara and Truelove, 1990). The use of a laser for debonding ceramic brackets has also been reported (Strobl et al., 1992). There are undesirable side effects to all of these methods. Mechanical debonding of ceramic brackets can cause enamel cracks, fractures, and flaking (AAO, 1989). Electrothermal or any other heat-producing devices have the potential to cause pulp irritation or possibly permanent damage. Thus, despite the employment of different methods for debonding, possible tooth or pulp tissue damage remains a concern to clinicians using ceramic brackets (Bishara and Fehr, 1997).

In summary, the superior aesthetics of ceramic brackets is their only advantage over stainless steel brackets, since their mechanical and chemical properties present a major problem in clinical use. However, the shortcomings that are associated with ceramic brackets should not detract from their use in orthodontic practice.

\section{BONDING CEMENTS}

\section{Resin Cements}

Direct bonding of orthodontic brackets became possible with the introduction of acid conditioning of enamel and the development of composite resin cements (Buonocore, 1955; Bowen, 1962). Currently, orthodontic bracket bonding is primarily accomplished with the use of composite resin cements. Almost all of these cements are based on the BIS-GMA formula that Bowen developed in 1962. He subsequently developed a Bisphenol A Glycidyl dimethacrylate (BIS-GMA) resin that proved to be considerably more stable than previous resins; it also had greater strength in the oral environment when filled with inert inorganic filler.

\section{Acrylic and Diacrylic Resin Cements}

Resin containing luting cements (RCLC) used for orthodontic bonding belong to two groups, acrylic and diacrylic resins (Reynolds, 1975). The constituents of acrylic resins are a 
methylmethacrylate monomer and an ultra fine polymer powder. Activation of polymerization of the resin is usually accomplished by the use of a catalyst (Reynolds, 1975). The catalyst is either the tertiary amine-benzoyl peroxide curing system, or the tri-nborane derivative (Reynolds, 1975). Acrylic resins form linear polymers. According to Gorelick et al. (1978) and Viazis et al. (1990) acrylic resin is a poor choice for luting cement for orthodontics because it lacks adequate strength. Diacrylic resins are based on an acrylic modified epoxy resin, which is referred to as BIS-GMA (Bowen, 1962). Diacrylic resins differ from acrylic resins because they may also be polymerized by cross-linking into a three dimensional network. This contributes to greater strength, low water absorption, and less polymerization shrinkage (Gorelick et al., 1978 and Phillips, 1982).

\section{Filler Content}

A composite material by definition consists of a mixture of two or more materials. Resin based composite materials have three major components: (Craig, 1997)

1. An organic resin matrix which is the chemically active component. The most commonly used monomer is BIS-GMA, which is derived from the reaction of Bisphenol-A and Glycidylmethacrylate.

2. An inorganic filler which is added to resin cements to increase their viscosity, reduce polymerization shrinkage, and allow for the thermal dimensional change of the set composite to approximate that of enamel.

3. A coupling agent, which bonds the filler and resin components to render acceptable mechanical properties to the composite.

It should also be noted that the inorganic filler increases the strength and wear resistance of resin cements (Craig 1977). The filler content of orthodontic resin cements, however, is lower than those used for restorative purposes. This is primarily to allow for easier removal of the remaining cement during debonding (Smith and Williams, 1982).

Acrylic and diacrylic resins exist as unfilled, low filler content, or high filler content resins. Filled resins are further subdivided based on the size of the particles, which can be large 
(macrofilled resins), or small (microfilled resins) (Sharma, 1999). Highly filled restorative composite resins contain $60 \%$ to $80 \%$ by weight of silica (glass filler), whereas the lightly filled composite resins contain about $28 \%$ by weight of silica. The orthodontic composite resins consist of $24 \%$ to $72 \%$ inorganic fillers (Smith and Williams, 1982). The two RCLCs tested in this investigation were Panavia $21^{6}$ and Transbond ${ }^{7}$. Panavia $21^{6}$ is a BIS-GMA twopaste low viscosity restorative composite resin with a filler content of over $70 \%$ by weight. This cement is auto-polymerized in the absence of oxygen (Rux et al., 1991). Panavia2 $1^{6}$ contains the adhesive monomer MDP (10-Methacryloyloxydecyl dihydrogen phosphate) which provides long-term bond strength between tooth structure and metal or silanated porcelain. (Rux et al., 1991). Transbond ${ }^{7}$ is a BIS-GMA based, no mix photo-polymerized microfilled orthodontic RCLC and is $80 \%$ filled by weight. Mechanically retentive bracket bases depend on the filler content of RCLCs to provide adequate bond strength. Highly filled resin composites appear to bond better to metal brackets (Dickinson and Powers, 1980; Powers, 1997). In addition, an increase in filler content increases its viscosity, which prevents slippage of the bracket on enamel surface before setting of the bonding material. It should be noted, however, that a RCLC with a very high filler content interferes with the wetting of the conditioned enamel which adversely affects the mechanical retention of the cement on to the tooth.

Composite filled resins are available in auto-polymerizing, photo-polymerizing, and dual cure systems. The following is a detailed description of each system.

\section{Auto-polymerizing and Photo-polymerizing Resin Containing Luting Cements}

\section{(RCLCs)}

Auto-polymerizing (chemically activated) RCLCs are divided to no-mix systems and twopaste systems (Zachrisson, 1994). The two-paste auto-polymerizing RCLCs require mixing of the two pastes for the initiation of polymerization reaction (setting reaction). These cements set in 30 to 60 seconds which limits their working time and necessitates quick removal of excess cement from the bracket periphery. Separate mixes are required for each bracket (Proffit, 1986). These two-paste composite resin cements also have a thicker viscosity, which reduces slippage of a bracket placed on a tooth. 
The no-mix auto-polymerizing composite resins consist of a composite paste and a liquid primer. A thin coat of primer is painted onto the bracket base and the conditioned enamel surface, after which the bracket base is coated with composite resin paste. As the bracket is pressed onto the enamel surface, the resin is activated from both the bracket and enamel surfaces. Excess flash is essentially un-reacted paste and it can be easily removed since it does not set. The no mix systems are less viscous and allow bracket slippage prior to polymerization of the cement.

The auto-polymerized RCLCs were the first system developed for orthodontic bracket cementation (Newman, 1968). Subsequently ultraviolet (UV) light sensitive resins with more rapid polymerization times were introduced as an alternative. The greatest advantage of the photo-polymerized cements is that they provide the clinician with ample time to accurately position brackets on the enamel surface and to remove excess material before photo-polymerization of the cement (Bishara et al., 2000). The wavelength of UV light is in the range of $200-400 \mathrm{~nm}$ and is just below that of visible light (Rock, 1974). UV activated cements could be manipulated indefinitely in the mouth until polymerization was initiated by exposure to UV radiation (Rock, 1974). The major disadvantage associated with the UV curing unit was leakage of UV radiation, which could reportedly produce skin cancer, eye damage, and erythema (Birdsell et al., 1977). In addition, the UV sensitive resin cements had a limited depth of cure that could not be improved (Rock, 1974 and Birdsell et al., 1977). The depth of cure is dependent on the composition of the material (amount of filler and particle size), the thickness of the mix, the opacity, and the intensity, position, and duration of curing of the light-source (Cook, 1980; McCabe and Carrick, 1989). As a result, the depth of cure of UV activated resin cements was limited due to attenuation of the UV radiation by the cement and the intervening tooth structure (Salako, 1979), and poor transmission through the tooth substance (De Saeytijd et al., 1994; Sargison et al., 1995). Another reason for limited depth of cure of UV activated systems was the increased amount of scattering at lower wavelengths (Ruyter and Oysaed, 1982).

Douglas et al. (1979) advocated the use of a visible light system to overcome the shortcomings associated with the UV light systems. This light system had curing units with 
a blue filter that produced blue light in the range of 400-500 $\mathrm{nm}$. Visible light sensitive resin cements were designed to absorb blue light and initiate polymerization. The advantage of the visible light curing system was the elimination of hazardous UV rays, an increase in the depth of cure, and a reduction in time of light exposure required for polymerization (Newman et al., 1983).

The composition of photo-polymerized RCLCs differs from the auto-polymerized RCLCs only in the activators and initiators. Photo-polymerized RCLCs use a diketone initiator and a reducing agent to initiate polymerization. The initiator is activated by light wavelengths in the range of 400-500 $\mathrm{nm}$. In comparison, auto-polymerized RCLCs are dependent on the mixing of the initiator and the activator for polymerization. (American Dental Association, 1985). Leung et al. (1983) demonstrated that post-irradiation hardening of a visible light activated system continues for up to 1 day.

There are conflicting reports in the literature when photo-polymerizing RCLCs are compared to auto-polymerizing RCLCs. Some studies report lower bond strengths for photo-polymerizing RCLCs compared with those achieved with auto-polymerizing RCLCs (King et al., 1987; Greenlaw et al., 1989; Ostertag et al., 1989). Other studies, however, (Joseph and Rossouw, 1990; Wang and Meng, 1992) have reported higher bond strengths for photo-polymerizing RCLCs than for auto-polymerizing RCLCs. Because of the different materials and the different methods used in their investigation direct comparison between these studies is not possible.

When comparing the failure rates of photo-polymerizing and auto-polymerizing RCLC, O'Brien et al. (1989) reported respective failure rates of $4.6 \%$ and $6 \%$ for photopolymerizing and auto-polymerizing RCLC. The values obtained by Armas et al. (1998) were $11.3 \%$ and $12 \%$. These differences may be attributed to the differences in both the physical and mechanical properties of materials tested, and the testing criteria used. The results of both studies, however, confirm the clinical acceptability of both photopolymerizing and auto-polymerizing RCLC bonding materials. In fact Sargison et al. (1995) noted comparable bond strength values for photo-polymerizing Transbond (3M/Unitek, CA) 
and auto-polymerizing Right-on (TP, Orthodontics). Based on these observations, Sargison et al. 1995 suggested that although light cannot penetrate a metal bracket, there seems to be sufficient light penetration through the periphery of the base to activate polymerization of the underlying cement.

\section{Dual Cured RCLCs}

In an attempt to address the problem of inadequate depth of cure, dual-cured RCLCs were developed. These materials contain both auto-polymerizing and photo-polymerizing systems (Sargison et al., 1995) so that complete polymerization is enhanced by the autopolymerizing component. As a result initial polymerization can be activated by the visible light source with auto-polymerization completing the setting reaction. In fact these bonding materials can also be left to auto-polymerize without any light exposure. $\mathbf{A}$ dual-cured system may be the most ideal bonding system for orthodontic purpose, since it confers both the advantages of extended working time and the continuation of polymerization when the light source is removed.

\section{Glass Ionomer Cements}

Despite their versatility, the use of RCLCs for bracket bonding has a number of drawbacks. Enamel loss could occur during prophylaxis (Thompson and Way, 1981), acid conditioning, (Brown and Way, 1978) as well as at the time of clean up during debonding (Pus and Way, 1980). In addition, enamel demineralization could develop within a month of bracket placement as a result of the accumulation and retention of plaque next to the bracket bases (O'Reilly and Featherstone, 1987; Mitchell, 1992). Glass ionomer cements are thought to have the potential means of addressing some of the shortcomings associated with the use of RCLCs in orthodontics.

Glass Ionomer Cements (GICs) possess the ability to adhere to non-precious metals, enamel, dentin and plastics (Hotz et al., 1977). It is also reported that less enamel damage occurs during clean up after debonding (Ostman-Andersson et al., 1993). According to Norevall et al. (1995), GICs can be more easily removed than RCLC at the time of debonding. Any cement remaining on the enamel surface can be desiccated by air drying which renders it 
more friable and removable (White, 1986). As a result, the likelihood of enamel damage being incurred both during and after debonding of GIC cemented brackets is less than that of those cemented with RCLCs. The use of GICs does not require the acid conditioning of enamel (Millett \& McCabe, 1996) since the cement bonds chemically to tooth structures i.e., enamel and dentin. GICs also have the ability to release (Fox, 1990; Ashcraft et al., 1997) and absorb fluoride (Hatibovic-Kofman and Koch, 1991; Creanor et al., 1994). Glass ionomer cements release considerable amounts of fluoride and they have been shown, in vitro, to prevent demineralization of enamel (Forss and Seppa, 1990). It has also been reported that a less cariogenic flora is found in plaque deposits adjacent to GICs (Hallgren et al., 1992). Wright et al. (1996) studied the effect of resin-reinforced glass ionomer cement (RRGLC) on the oral microflora. Their results showed that levels of Lactobacilli and S.mutans (cariogenic bacteria) around brackets bonded with the RRGIC were reduced when compared with the conventional diacrylate resin cements.

GICs were introduced by Wilson and Kent in 1972 and they consists of following constituents (Crisp et al., 1976):

1. Poly alkenoic acid, commonly a homo- or co-polymer of acrylic acid.

2. An ion leachable aluminosilicate glass to provide ions to cross-link the chains. These glass particles are embedded in an insoluble hydrogel matrix.

3. Water as a reaction medium.

4. Tartaric acid to improve working and setting characteristics.

There are three types of glass ionomer cement currently on the market: conventional, water hardening, and the most recently introduced, the resin-reinforced glass ionomer cement (RRGIC) (White, 1986; Antonucci et al., 1988).

The setting reaction of conventional GIC involves an acid base reaction between the aluminosilicate glass and polyacrylic acid components (Wilson and McLean 1988). Protons from the acid penetrate the glass powder to release calcium and aluminum ions, initiating a prolonged, two-phase setting reaction. Calcium ions bind to polyacrylic acid producing gelation and initial adhesion to tooth structure. Aluminum ions then contribute to the 
subsequent hardening stage as more rigid cross-linking occurs with the formation of aluminum polycarboxylates (Wilson and McLean, 1988). It is during the first phase when calcium salts predominate, that conventional glass ionomers are extremely sensitive to moisture contamination and dehydration (Swift, 1986). Conventional glass ionomer cements have reportedly shown decreased bond strength when used to bond orthodontic brackets when compared to resin cements (Cook, 1990; Fajen et al., 1990; Rezk-Lega and Ogaard, 1991).

The second generation or water hardening GICs contain the same acids as conventional GICs either in a freeze dried powder form or as an alternative powdered copolymer of acrylic and maleic acids. These dried powders are supplied blended with the glass powder and require only distilled water to activate the setting reaction. Hence the name water hardening GIC (Prosser et al., 1984). These types of GICs are used to cement orthodontic bands.

\section{Resin-Reinforced Glass Lonomer Cements}

The new generation of GICs are hybrid cements containing water base GIC with $11.25 \%$ resin (Demke R. Material product data sheet, 1996). These hybrid GICs set partly via an acid reaction and partly via either photochemical or chemical polymerization (Bourke et al., 1992). Since these materials contain both glass ionomer and resin components, they differ considerably in their physical and chemical properties from the conventional GICs (Millett and McCabe, 1996). These cements combine the advantages of conventional GICs with the mechanical and physical properties of composite resin cements (Beress et al., 1998). A photo-polymerized, hybrid glass ionomer orthodontic cement, Fuji Ortho LC was introduced in 1994 by GC Orthodontics (Coups Smith, 1997). It is the manufacturers claim that this cement allows bonding to non-conditioned enamel in a moist environment. Two reactions take place on polymerization of this cement: the normal glass ionomer cement acid-base reaction that occurs when the material is mixed as well as a photochemical polymerization that occurs on exposure to visible light (Sidhu and Watson, 1995). RRGICs are supplied as powder and liquid. The powder comprises ion leachable fluoroaluminosilicate glass (Craig, 1997). The complex monomer liquid contains polyacrylic acid, water, and a photo- 
polymerized monomer such as hydroxyethyl methacrylate (HEMA) or BIS-GMA. RRGICs are formed by a combination of the acid-base components of conventional GICs and the addition of resins. Monomers such as BIS-GMA and HEMA are used to facilitate the chemical reaction. In place of some of the water, HEMA is incorporated into the glassionomer composition. This provides for a dual setting mechanism that is accelerated by the light polymerization of resin monomers (Antonucci, 1988 and Nouri, 1997). A moist environment is required for all the reaction steps but, it should be noted, the presence of too much water may dissolve the reactants thus preventing the agglomeration of the setting matrix (Wilson, 1990). Resin-reinforced glass ionomer cements that possess photochemical setting reactions, appear to have a reduction in sensitivity to moisture, since the resin network reduces the diffusion of external water into the setting cement (Cho et al., 1995; Shen and Grimaudo, 1994). During bonding the enamel surface needs to be moistened with either water or saliva in order to avoid enamel desiccation, as this will adversely affect bond strength (Silverman et al., 1995). During mixing, an incorrect powder/liquid ratio will affect RRGICs clinical performance because of its effect on the physical and chemical properties of the set material. For example, a watery mix will result in an increase in the amount of the hydrophilic resin (HEMA) that increases the potential for greater water uptake and weakening of the final material (Wilson, 1990). On the other hand, a thick mix will result in increased viscosity that will reduce its ability to wet the substrate (enamel surface) effectively. The result of this may be a decrease in the ultimate bond strength. In addition, an adequate thickness is required to reduce the potential of the bracket float problem associated with a thinner mix.

In comparison with the conventional glass ionomers, the resin-reinforced glass ionomer cement is believed to have improved resistance to desiccation and acid attack. The bond to enamel and dentin is also enhanced, since the resin component imparts additional tensile strength to the set cement (van Noort, 1994). 


\section{Conditioned and Non-Conditioned Enamel Surfaces}

The enamel treatment required with the use of Fuji Ortho LC is conditioning with a 10\% aqueous solution of polyacrylic acid. This acid does not dramatically change the enamel surface compared to phosphoric acid; it simply cleans the surface and promotes a suitable bonding substrate for application of the cement (Todo et al., 1997). The early reports by Silverman et al. (1995) on the clinical performance of Fuji Ortho LC were promising, with bracket failure rate of about 3 per cent recorded; the enamel surface was not conditioned and the observation period, appeared to be about 8 months. It was also suggester that enamel acid conditioning was not necessary, unless a strong bond was required (Silverman et al., 1995).

Fricker (1994), in a clinical trial of 10 cases, reported no significant difference in failure rates of orthodontic brackets cemented with light cured resin-reinforced Fuji II LC (GC Dental Industrials, Tokyo, Japan) and those cemented using composite resin (System 1+, Ormco Corp, Glendora, Calif). Using Fuji Ortho LC as a bracket cementing agent, Powers et al. (1996) reported bond strengths to non-conditioned enamel ranging from 8 to $25 \mathrm{MPa}$. Beress et al. (1998) tested Fuji Ortho LC under different surface treatment conditions when bonding metal brackets to human enamel. They reported mean bond strength of $17.69 \pm 4.6$ MPa for conditioned moist environment, however, the bond strength dramatically decreased when the enamel surface was not conditioned (5.27 $\pm 2.15 \mathrm{MPa})$. Bishara et al. (1998b) and Millett et al. (1999) reported that Fuji Ortho LC cement, when used on conditioned enamel contaminated with a thin film of water or saliva, has a similar but slightly lower strength compared to that of traditional photo-polymerized RCLC. Their findings also indicated that conditioning the enamel surface was a critical variable that affected shear bond strength, as well as bond failure location, when the Fuji Ortho LC cement was used. Coups Smith (1997) recommended the use of $10 \%$ polyacrylic acid conditioner prior to bonding with either GC Fuji Ortho (auto-polymerizing RRGIC), or Fuji Ortho LC (photo-polymerizing RRGIC). In a study comparing the shear bond strengths of RRGICs following incubation periods of 24-hours and 30-days, Lippitz et al. (1998), also reported shear bond strengths comparable to those of the resin cements when the enamel was conditioned with $10 \%$ polyacrylic acid solution. They noted significant reductions and less consistency in bond 
strengths when the enamel was not conditioned. Furthermore, with the omission of enamel conditioning, the site of bond failure shifts from the bracket base-cement interface to the enamel-cement interface thus confirming the weaker bond between the enamel and the cement (Bishara et al., 1998b). Despite the manufacturer's claim these observations confirm that in order to attain a reliable bond strength the acid conditioning steps should be followed prior to cementation of brackets with RRGICs.

Flores et al. (1999) tested the SBS of metal brackets to acid conditioned and nonconditioned enamel surfaces using photo-polymerized Fuji Ortho LC and Transbond cement. The enamel was conditioned for 20 seconds with $37 \%$ orthophosphoric acid for both cement types. In this study, the investigators did not use the recommended $10 \%$ polyacrylic acid conditioner for Fuji Ortho LC. Their results showed that shear bond strength was dependent on enamel surface treatment. When bonding with Fuji Ortho LC a significant increase in shear bond strength was observed with the conditioned group $(15.8 \pm 2.08 \mathrm{MPa})$ as compared to non-conditioned group $(6.4 \pm 3.40 \mathrm{MPa})$. The bond strength resulting from Transbond (14.7 \pm 4.22$)$ and Fuji Ortho LC $(15.8 \pm 2.08 \mathrm{MPa})$ for the conditioned groups were comparable. With respect to enamel surface alteration following debonding, the nonconditioned group demonstrated no enamel changes. However, there was less damage to enamel surface, as indicated by scanning electron microscopy, when Fuji Ortho LC (conditioned) was compared to Transbond (conditioned) group. They concluded, therefore, that the use of Fuji Ortho LC for bracket bonding onto acid conditioned (37\% orthophosphoric acid) enamel was a favorable option because debonding strengths were significantly increased and there was less iatrogenic damage to the enamel surface when compared to Transbond (conditioned) group.

Bishara et al. (2000) studied the effects of altering the type of enamel conditioner on the SBS of RRGICs within half an hour after bonding the bracket to the enamel. The half an hour waiting period used was based on the results of their previous study (Bishara et al., 1999b). In this study, the authors demonstrated low bond strengths following half hour bonding with RRGICs in conjunction with polyacrylic acid conditioner. The experimental sample consisted of four groups: group I) $10 \%$ polyacrylic acid conditioner and Fuji Ortho 
LC cement, group II) $20 \%$ polyacrylic acid and Fuji Ortho LC cement, group III) $37 \%$ phosphoric acid, and Fuji Ortho LC cement and group IV) 37\% phosphoric acid and Transbond cement. These investigators recorded clinically acceptable SBS in the two groups that had been conditioned with $37 \%$ phosphoric acid. The bond strength of the RRGICs conditioned with $10 \%$ polyacrylic acid $(0.4 \bullet 1.0 \mathrm{MPa})$ was significantly lower than the group conditioned with $20 \%$ polyacrylic acid $(3.3 \pm 2.6 \mathrm{MPa})$. As a result, they concluded that glass ionomer cement had significantly low initial bond strength in the first half hour after bonding. They further suggested that ligating archwires within that time frame could result in an unacceptable rate of bracket failure. Their recommendation was that in order to safely tie archwire into brackets bonded with RRGIC within half an hour of bonding, clinicians should consider conditioning with $37 \%$ phosphoric acid instead of polyacrylic acid. Another suggested alternative was to tie archwires 24-hours after bonding, since by that time bond strengths were shown to have reached an optimal level to withstand orthodontic forces. The investigators acknowledged the inconvenience incurred by both the clinicians and the patient, but it was their opinion that the benefits from fluoride release probably counterbalanced such an inconvenience. The cumulative evidence produced by these current studies is that the presence of only chemical bond (RRGIC to non-conditioned enamel) does not appear to provide clinically sufficient SBS. The addition of a mechanical bond to enamel through enamel conditioning is required to provide clinically acceptable bond strengths (Meehan, 1999).

\section{TYPES OF ENAMEL SURFACE CONDITIONERS}

\section{Acid Conditioning}

Acid etching (conditioning) was initially introduced in 1955 by Buonocore. He demonstrated a markedly increased retention of methyl methacrylate resins to enamel when their application was preceded by enamel conditioning using $85 \%$ phosphoric acid for 30 seconds. Subsequently, the optimum concentration of acid to produce a consistent evenly distributed, and optimal depth etch pattern was reported to be in the range of 30 to $50 \%$ (Buonocore, 1955; Retief, 1974; Silverstone et al., 1975). Acid conditioning modifies the enamel surface, allowing an intimate micro mechanical bond between enamel and the resin component of the RCLC. The latter has no intrinsic adhesive qualities to the enamel. The 
conditioning process increases surface roughness of the enamel and hence the bonding area by removing the hydroxyapatite from the enamel surface. Thus, the surface characteristics of enamel are changed due to preferential dissolution between the prism periphery and its core. Following acid conditioning, the enamel surface assumes a microscopic honeycomb lattice appearance (Craig, 1997). Wickwire and Rentz (1973) estimated the surface layer of enamel lost during conditioning to vary between 10 and $30 \mu \mathrm{m}$. The acid also has the effect of raising the surface energy of enamel from a low-energy hydrophobic surface to a highenergy hydrophilic surface. This surface modification increases enamel surface tension and wettability by the cement (Retief, 1978). This facilitates the flow of the resin material over the enamel surface, allowing greater penetration of resin tags into the undercuts of the conditioned surface. After polymerization, the adhesive resin tags form a tightly interlocking mechanical bond with the conditioned enamel (Retief, 1978). Buonocore et al. (1978) showed that the depth of penetration of the resin tags reached up to $50 \mu \mathrm{m}$. This resin is thought to remain on the enamel after debonding, and this could affect plaque retention, susceptibility to caries and discoloration (Waveren et al., 2000).

Since phosphoric acid conditioning may potentially damage enamel integrity, numerous investigations have been conducted to assess the merits of alternative treatments such as reducing acid concentration and time, use of maleic acid (Triolo et al., 1993; MacColl, 1995; Olsen et al., 1997; Urabe, 1997), and polyacrylic acid (Smith and Cartz, 1973). Investigations concerning reducing either the concentration of the acid or the conditioning time have concluded that varying phosphoric acid concentration from $5 \%$ to $37 \%$ (Barkmeier et al., 1987; Legler et al., 1989; Sadowsky et al., 1990) or reducing the conditioning time from 60 to 15 seconds (Barkmeier et al., 1987; Legler et al., 1989; Sadowsky et al., 1990) and even 10 seconds (Olsen et al., 1994) does not significantly affect bond strength. It has been reported, however, that reducing the conditioning time, within certain limits, to 5 seconds results in inadequate bond strength (Olsen et al., 1994). Thus, reducing acid concentration and conditioning time within certain limits, produces less tooth damage whilst still yielding adequate bond strength. According to Wang and Lu (1991) the shorter the conditioning time, the less the depth of enamel loss and the fewer enamel fractures during debonding. Triolo et al. (1993) using $10 \%$ maleic acid as enamel 
conditioner found that it provided bond strength essentially equal to that of $37 \%$ phosphoric acid when bonding brackets with RCLCs. In this study, the scanning electron microscopy of the enamel treated with $10 \%$ maleic acid and $37 \%$ phosphoric acid revealed a similar morphologic pattern but the depth of the etched surface was significantly less with maleic acid. MacColl (1995) compared shear bond strengths of metal brackets bonded to bovine enamel using four different conditioners: 37\% Phosphoric acid (aqueous solution and gel), $10 \%$ maleic acid (aqueous solution and gel) for 20 seconds. His results demonstrated that conditioning with aqueous maleic acid (10\%) was associated with the highest shear bond strength. There was no statistically significant difference between the other three acid types used.

Acid conditioning, in general, may initiate enamel decalcification by removing highly mineralized fluoride rich surface enamel; it may also promote enamel fracture during debonding due to adherent cement tags within the microporosities. The clean-up procedure of the cement after debonding may remove up to 55 microns of surface enamel (Fitzpatrick and Way, 1977). Therefore, using phosphoric acid for acid conditioning carries a potential risk for significant enamel loss, either during conditioning or following removal of penetrated cement. As a result, any bonding system that can produce adequate bond strengths without any significant enamel surface alteration, subsequent decalcification, and possible enamel fracture will be a superior system.

\section{Crystal Growth}

Crystal growth, introduced by Smith and Cartz (1973), is an alternative method of enamel surface preparation. Smith and Cartz (1973) showed that polyacrylic acid containing residual sulfate ions reacied with the enamel surface to produce a deposit of white spherulitic crystalline salcium sulfate to which the adhesive resin bonds. Smith and Cartz (1973) identified these crystals as calcium sulfate dihydrate (gypsum). According to Smith and Cartz (1973) the carboxyl groups in the long chain polyacrylic acid molecules have the ability to chelate to calcium in the mineral phase of tooth structure, resulting in an adhesion. The formation of these depended mainly on the sulfate ion concentration in the polyacrylic acid solution. Purified polyacrylic acid produced only slight etching of the enamel surface; 
whereas polyacrylic solutions that contained residual sulfate ions produced not only slight etching of the enamel but also a crystalline deposit that bonded firmly to the enamel surface and resisted mechanical removal (Smith and Cartz 1973). These investigators also demonstrated that the maximum density of the long, needle-shaped crystals growing on the enamel surface was found to occur after conditioning for 4 minutes with $40 \%$ polyacrylic acid. With this method of enamel conditioning, the resin penetrated the deposited crystals on the surface rather than the enamel itself. The method of crystal growth for bonding has a few advantages over the etching (conditioning) technique with the phosphoric acid. These advantages are: (1) minimal damage to the enamel surface, (2) easier debonding and enamel cleanup, (3) minimal loss of outer fluoride rich enamel layer, and (4) few if any resin tag remnants after debonding (Maijer and Smith, 1986). Bishara et al. (2000) compared the etch pattern of enamel surface when it was etched with $37 \%$ phosphoric acid, 10 polyacrylic acid, and $20 \%$ polyacrylic acid. When the enamel surface was examined under an electron microscope, phosphoric acid conditioning produced a much deeper etch (rougher enamel surface) than the polyacrylic acid. It has been demonstrated that the use of crystal growth enamel conditioning with polyacrylic acid, significantly increases the incidence of bond failure at the enamel-cement interface, but the bond actually fails within the crystals and not at the enamel surface. As a result, the incidence of enamel fracture is decreased (Beech, 1972; Maijer and Smith, 1979). Maijer and Smith (1979) tested the value of this crystalline interface as an enhancer of the mechanical retention of orthodontic brackets. The results of their study showed that the crystalline interface produced tensile bond strength equivalent to that of a conventionally acid-conditioned enamel surface. However, other investigators (Maskeroni et al., 1990; Artun and Bergland, 1984; Farquhar, 1986; and Burkey, 1985) found that bond strengths with the use of crystal growth conditioning were significantly weaker than with the conventional acid conditioning techniques.

Bishara et al. (1993) compared various conditioner-cement combinations and indicated that, in general, the use of polyacrylic acid as an enamel conditioner resulted in a $30 \%$ reduction in bond strength as compared with the use of phosphoric acid. However, despite this reduction, the bond strengths were acceptable for orthodontic purposes. 


\section{Adhesive Primer}

Conventionally, after the enamel surface is conditioned with phosphoric acid, an intermediate unfilled low viscosity liquid resin (adhesive primer) is applied in order to thoroughly wet the enamel surface so that the bond strength between enamel and the resin cement is maximized. Application and curing of this adhesive primer results in resin tags that extend into the microporosities that are produced by acid conditioning. These resin tags bond the composite cement mechanically to enamel (Retief, 1978). An adhesive primer is a multifunctional monomer with a hydrophilic end that wets and bonds to tooth structure and a hydrophobic end that reacts with the double carbon bonds of the resin cement. Weisser (1973) and Reynolds (1975) advocated the use of low viscosity adhesive primers to ensure adequate wetting of the enamel surface. However, research has shown that the application of a layer of unfilled adhesive resin to the conditioned tooth surface prior to placement of the composite resin cement and bracket does not increase the bond strength and can, therefore, be omitted (O'Brien et al., 1991; Wang and Tarng, 1991). Tang et al. (2000) recently performed a retrospective clinical study to evaluate the retention of metal orthodontic brackets bonded without adhesive primer. In both the test and control groups the enamel was conditioned using $37 \%$ phosphoric acid. In the experimental group $(n=37)$ Phase II resin cement (two paste auto-polymerized resin cement, Reliance, Itasca, IL) without adhesive primer was used to bond brackets to patients' maxillary teeth. Brackets in the control group $(n=37)$ were bonded to the teeth with Phase II and adhesive primer. The results showed that the exclusion of an adhesive primer from an auto-polymerized two paste bonding resin cement appeared to have no detrimental clinical effect. Despite these observations, however, clinicians continue to utilize adhesive primers in conjunction with the RCLCs in order to comply with manufacturer's recommendations.

A recent development in the field of bonding is the use of innovative self-etching acidic primers. These materials serve simultaneously as conditioner and primer and do not have to be rinsed off. The acidic part of the primer is neutralized at some point by the calcium and phosphate ions released during demineralization. Demineralization is, therefore, selflimiting in that the high concentration of these ions tends to limit further dissolution of hydroxyapatite (Wang and Hume, 1988). The acidic primers form a continuum between the 
tooth surface and the cement material by the simultaneous demineralization and resin penetration of the enamel. The advantages of acidic primers are simplified bonding procedures and improvement in both reduced time and cost effectiveness to the clinicians and thus indirectly to patients (Bishara et al. 1998a).

The use of acidic or self-etching acidic primers for orthodontic purposes has been evaluated in two different studies by Bishara and colleagues (Bishara et al., 1998a and 1999a). The study conducted in 1998 showed that the use of an acidic primer to bond orthodontic brackets to the enamel surface provided clinically acceptable SBS (11.8 $\pm 4.1 \mathrm{MPa})$ when used with a highly (77\%) filled RCLC (Panavia21). The shear bond strength was significantly lower $(5.9 \pm 5.6 \mathrm{MPa})$, however, when a lightly (10\%) filled RCLC was used (Clearfil Liner Bond, J.C. Morita, Kuraway, Japan). The use of an acidic primer decreased the amount of cement left on the tooth after debonding. This observation was also illustrated by examining scanning electron micrographs (SEM) of the enamel surfaces. The SEM for phosphoric acid conditioned enamel showed thick and uniform resin tags; whereas the resin tags for the self-etching acidic primer treated enamel were thin and less uniform. The latter observation supports the finding that there is a weaker bond between the enamel and the cement with the result that less cement is left on the tooth after debonding. The results of the Bishara et al. (1999a) second study did not endorse the use of an acidic primer, but it must be noted that they used different cements. In this latter study, two types of adhesive were compared with different types of conditioners and primers. The results indicated that the resin/phosphoric acid adhesive system provided the strongest $(10.4 \pm 2.8 \mathrm{MPa})$, and the glass ionomer adhesive system a significantly lower SBS (6.5 $\pm 1.9 \mathrm{MPa})$. The lowest shear bond strength was noted when the acidic primer was used with an orthodontic cement (Transbond). Based on these observations, and the introduction of self-etching acidic primers more research is warranted to test the compatibility of self-etching acidic primer + Panavia21 using metal, and ceramic brackets. 


\section{STORAGE TIME}

A review of the pertinent research shows that the majority of bonding studies use 1-hour, 24-hours, 7-days and 28-30 days (Rix et al., 2001; McCourt et al., 1991, Meehan et al., 1999, Lippitz et al., 1998, Lalani et al., 1999) as storage time prior to shear bond strength (SBS) testing. Sharma (1999) tested six different metal bracket bases bonded with Transbond cement after 1 and 24-hours of storage time. The results indicated a statistically significant difference between the SBS of data collected at 1-hour versus data collected at 24-hours. Time significantly increased mean SBS, however, this difference was not clinically significant because 5 out of 6 bracket types tested achieved clinically acceptable bond strengths after 1-hour. The conclusion was that, the data arising from 24-hours testing provides relevant clinical information and that there is no need to wait for 24-hours prior to placement of orthodontic wires and subsequent force application. These results are in disagreement with those of Greenlaw et al. (1989) who recommended that 24-hours should elapse before force application.

Chamada and Stein (1996) tested the immediate shear/peel bond strengths produced by a photo-polymerized bonding system (Transbond) in an in vitro study. They also compared the shear/peel bond strengths over a 24-hours period with those obtained by an autopolymerized orthodontic bonding system (Concise). The times at which the bond strengths were tested were $0,2,5,10$ and 60 -minutes and 24-hours after activation with the photopolymerized cement. For the auto-polymerized cement, the time intervals for testing were 2 , 5,10, 60-minutes and 24-hours after activation. Their findings showed that photopolymerized cement (Transbond) produced initial bond strengths after activation that was of sufficient magnitude to withstand the immediate application of forces. Their findings were in agreement with those of Tavas and Watts (1984) and Sharma (1999). In the study of Tavas and Watts it was shown that the initial bond strength of the cements used was $60 \%$ to $70 \%$ of the bond strength realized at 24-hours which once again was adequate to withstand immediate application of orthodontic and chewing forces. In their study, initial bond strength was low but it was shown to increase subsequently. 
Currently, there is no consensus on the effects of long term storage on the shear bond strength of orthodontically bonded brackets (Bishara et al., 1975; Low and von Fraunhofer, 1976; Johnson et la., 1976; Yamaguchi et al., 1989; Meng et al., 1995). Meng et al. (1995) conducted a study to determine the effects of water immersion on orthodontic resins used to bond metal brackets to human premolars. The results showed that there was a significant reduction in bond strength after one day. They speculated that the polymer matrix of composite resin cements absorb and desorb water over time causing the hydrolytic degradation of the inorganic fillers, thus, resulting in reduced bond strengths. According to Chamda and Stein (1996), shear bond strength increases over time due to the continuation of polymerization of the composite resin under the bracket base. Wilson and Mclean (1988) reported that GICs like composite resins slowly increase their strength over time thus leading to increased shear bond strengths. Yamaguchi et al. (1989) showed that there was no significant difference in shear bond strength when bonded specimens were stored in water for 24-hours to 7-days.

Recently, Mahal (2000) studied the effects of long term storage (180-days) on shear bond strength and ARI scores. Metal (Speed), ceramic (Transcend) and polycarbonate (SpiritMB) brackets were bonded to bovine enamel using Phase II composite resin and GC Fuji Ortho cements (auto-polymerizing). The long-term storage period consisted of shear bond testing at 24-hours, 7-days, and 180-days. The results indicted that in general long-term storage has a significant effect on the SBS and this effect was in turn dependant on the bracket type, and the cement used. In the Mahal (2000) study long-term storage (180-days) did not have a significant effect on the fracture pattern of brackets.

\section{HUMAN AND BOVINE ENAMEL}

One of the problems associated with bond strength testing is the limited availability of noncarious human teeth. Bovine mandibular incisors teeth are considered a suitable alternative to human teeth in bonding studies, and are used not only due to the limited availability of human teeth but also because of the increased concern of the infection hazards from human teeth (Rueggberg, 1991). Bovine teeth are readily available and their enamel is quite similar 
to human enamel. On a histochemical and anatomic basis the teeth of all mammals appear to be similar (Leichester, 1949; Fujita, 1957; Suga et al., 1971). Despite the general similarities, however, some differences have been noted. Bovine enamel and dentin form more rapidly than human enamel so that bovine enamel has larger crystal grains and more lattice defects than human enamel (Moriwaki et al., 1968). This may contribute to the observation of the lower critical surface tension in bovine enamel compared with human enamel (Oesterle et al., 1998; Yu and Chang, 1966). This difference between the critical surface tension of bovine and human enamel has been implicated as the reason for a slight enamel bond reduction when using bovine enamel (Nakamichi et al., 1983). Nakamichi et al. (1983) also investigated the suitability of bovine teeth as an alternative to human teeth in bonding tests, using seven different cements. The results showed that the adhesive bond strength to enamel was not statistically significantly different between the bovine and human teeth although the mean values were reported to be slightly lower with bovine teeth. Fowler et al. (1992) showed that bond strength measurements in both shear and tensile mode obtained with human and bovine enamel were essentially comparable. More recently, Oesterle et al. (1998) re-examined the suitability of bovine enamel in bonding studies. In this study, the strength of the enamel bond of a photo-polymerized orthodontic cement was compared between deciduous bovine, permanent bovine, and human enamel. The results showed that the bond strength to bovine enamel was significantly weaker than that to human enamel, and that the bond strength to deciduous bovine enamel was significantly greater than to permanent bovine enamel. The difference in bond strength between permanent and deciduous bovine enamel was attributed to the fact that permanent bovine incisors have larger undulations on the facial surface than both human incisors and deciduous bovine incisors. This uneven enamel surface is usually polished to create a smooth surface prior to bonding and this may account for the decreased bond strength of permanent bovine compared to deciduous bovine incisor (Oesterle et al., 1998). Despite these observations, bovine enamel can still be successfully used to study enamel bond strength (Fox et al., 1994, Oesterle et al., 1998). Thus, the current view is that orthodontic brackets bonded to bovine enamel will perform almost in the same way as they do to human enamel (Fox et al., 1994). 


\section{BOND STRENGTH TESTING}

The variables affecting bracket bond strength to enamel are the bracket base configuration, the cement used, and the type of enamel conditioner (Bisahra et al., 1993). Studies have also shown that storage time and water immersion does affect bond strength of brackets to enamel. Bond strength tests are used to assess the in vitro performance of dental bonding systems and also to predict their behavior (Bagnall, 1989). In the majority of orthodontic invitro studies, shear and /or tensile bond strengths are determined in order to assess the potential performance of the bracket and the bonding systems during clinical application. Ferguson et al. (1984), and Tavas and Watts (1979) both advocated the use of shear/peel testing to represent the type of forces encountered in the clinical situation. Fowler (1992) identified three variables associated with adhesion testing: (1) the test mode, shear and tension; (2) design of the testing apparatus; and (3) tooth substrate, human and bovine. When comparing the types of bond failure produced by shear and tensile testing, it was found that tensile specimens failed cohesively whereas the shear specimens failed at the enamel cement interface. As a result, it was concluded that the values obtained from tensile testing did not represent the bond to enamel but rather showed the bulk strength of the luting material (cohesive failure). Fowler also demonstrated that the shear test might have an advantage over tensile test in that it appears to be more likely to produce failure at the enamel-cement interface. According to Thanos et al. (1979), however, the best bracket basecement combination cannot be selected on the basis of one test alone.

The bond between the enamel and the bracket base should be strong enough to withstand orthodontic and chewing forces while still allowing debonding of the bracket without injury to tooth structure. Reynolds (1975) suggested that minimum bond strength of 5.9 to $7.8 \mathrm{MPa}$ was adequate for most clinical orthodontic needs. It was postulated that clinical success could be achieved with cements that provide in vitro bond strengths of approximately 5.0 MPa. If the bond between the cement and the enamel is stronger than the enamel itself, the enamel will fracture during debonding. An investigation by Retief, (1974) on bond failure at the enamel-cement interface indicated that enamel fractures can occur on debonding with bond strengths as low as 9.7 MPa. As a result the use of bracket base-cement combinations 
that can result in bond strengths that are significantly greater than $9.7 \mathrm{MPa}$ should be approached with caution.

\section{LOCATION OF BOND FAILURE}

Clinically, bond failures occur in the presence of excessive shearing, tensile, or torque forces. Bond failures can be divided into two types: cohesive failure and adhesive failure. Cohesive failure can occur within the tooth, bracket, or the cement. Adhesive failure occurs at the tooth-cement, and the bracket base-cement interfaces (Compton et al., 1992; Wang and Meng, 1992). According to Powers et al. (1997), the most important bonding information for orthodontists is obtained by isolating these interfaces and examining the different bond strengths resulting from various conditioner-cement-bracket base combinations.

Bond failure location is an important factor when considering chair time and potential damage to the pulp and the enamel surface (Katona, 1997). Following a critical review of bond strength testing in orthodontics, Fox et al. (1994) noted that the ideal location for a clinically acceptable bond to fail is at the enamel-cement interface since this would make bonding and subsequent debonding much easier. However, the enamel-cement interface is now considered an undesirable bond failure location, because of the increased risk for damage to the enamel. To maintain enamel integrity, the ideal bond failure location in debonding should be between the bracket base and the cement (Sinha et al. 1995), although bracket cement interface debonding increases the difficulty of enamel clean up (Brown, 1978; Pus, 1980). As a result, clinicians must take great care when removing remaining cement following debonding to avoid gouging, scratching, or pitting of the enamel surface.

The Adhesive Remnant Index (ARI), formulated by (Artun and Bergland, 1984), is used to quantify the amount of cement left on the tooth following debonding of the bracket. The ARI score is also used to define the sites of bond failure between the enamel, the cement, and the bracket base. The ARI consists of a 4-point scale of 0-3: a score of 0 indicates no cement left on the tooth, 1 indicates less than half of the cement left on the tooth, 2 indicates 
more than half of the cement left on the tooth, and 3 indicates all of the cement left on the tooth including a distinct impression of the bracket base.

Table 1: ARI Scoring Index (Artun and Bergland, 1984)

\begin{tabular}{|l|c|}
\hline \multicolumn{1}{|c|}{ Description } & ARI Score \\
\hline No cement left on the tooth & 0 \\
\hline Less than half of the cement left on the tooth & 1 \\
\hline More than half of the cement left on the tooth & 2 \\
\hline $\begin{array}{l}\text { All of the cement left on the tooth plus a distinct } \\
\text { impression of the bracket base }\end{array}$ & 3 \\
\hline
\end{tabular}




\section{OBJECTIVES OF THE STUDY}

- To identify combinations of bracket bases and cements that can provide clinically acceptable SBS which result in minimal or no damage to the enamel during debonding

- To ascertain the influence of bracket base design, cement type, enamel conditioning and long-term storage on SBS

- To ascertain the influence of bracket base design, cement type, enamel conditioning and long-term storage on ARI

- To determine if there is any association between ARI and SBS

- To investigate if there is a systematic relationship between different bracket base designs and particle size of various cements

In this study mean SBS of $8 \pm 3 \mathrm{MPa}$ and Adhesive Remnant Index (ARD) scores $\geq 2$ were deemed to be clinically acceptable.

\section{HYPOTHESIS}

Not all orthodontic bracket bases are clinically compatible with all the currently available cements. 


\section{OPERATIONAL DEFINITIONS}

Clarity': Polycrystalline ceramic bracket (3M/Unitek Dental Products, 2724 South Peck Road, Monrovia, CA 91016 USA).

Transcend²: Polycrystalline ceramic bracket (3M/Unitek Dental Products, 2724 South Peck Road, Monrovia, CA 91016 USA).

Time ${ }^{3}$ : Machined, integral metal bracket with microetched base with mechanical undercuts (American Orthodontics, 1714 Cambridge Ave, P.O. Box 1048, Sheboygan, Wisconsin 530821048).

Speed4: Metal bracket with 60 gauge, microetched foil mesh of horizontal and vertical configuration (Strite Industries, 298 Shepard Ave, Cambridge, Ontario N3C IV1).

Fuji Ortho LC $\mathbf{C}^{5}$ : Photo-polymerising resin reinforced glass ionomer cement (GC Corporation, Tokyo, Japan \& distributed by GC American Inc, Chicago, IL).

Panavia216: Photo-polymerizing restorative cement (J Morita USA Inc, Tustin, California).

Transbond7: Photo-polymerizing, diacrylate, highly filled, adhesive paste (3M Unitek Dental Products, 2724 South Peck Road, Monrovia, CA 91016 USA).

Digitek Image Processing System Series $100^{8}$ : Morphometric computer program (Digitek Co., Brooklyn, NY).

Plasticene': Art Works (Toronto, Ontario, Canada).

180-600 Grit Silicon Carbide paper ${ }^{10}$ : (Buehler Ltd., 41 Waukegan Rd., Lake Bluff, IL).

37\% Orthophosphoric Acid" : 37\% phosphoric acid (Reliance orthodontic Products Inc., P.O. Box 678 Itasca, IL 60143).

Heliobond 12: Poto-polymerising adhesive primer (Vivadent/Ivoclar, 23 Hanover Dr., St. Catherines, Ont., Canada L2W 1A3).

Semi-Adjustable Hanau Articulator ${ }^{13}$ : (Teledyne Hanau, Buffalo, NY, 14225).

Visible Curing Light Unit ${ }^{14}$ : Caulk The Max (Caulk Dentsply, L.D. Caulk Division, Milford, DE).

Visible Curing Light Meter ${ }^{15}$ : Cure Rite (Caulk Dentsply, L.D. Caulk Division, Milford, DE).

Electric Dryer ${ }^{16}$ : 120V Handi-Dri, Lancer pacific, P.O. Box 819 Carlsbad, CA 92008).

10\% Polyacrylic Acid ${ }^{17}$ : (GC Corporation, Tokyo, Japan \& distributed by GC America Inc.).

ED Primer ${ }^{18}$ : Self-etching acidic primer (J Morita, USA Inc, California). 
Instron Universal Testing Machine ${ }^{19}$ Model 4301 (Instron Corporations, 100 Royal Street, Canton, Mass, 02121).

Light Microscope ${ }^{20}$ (Bausche \&Lomb, cat. No.31-35-47).

Hitachi S-250021 : A scanning electric microscope (SEM) (Mito City, Japan). 


\section{MATERIALS AND METHODS}

\section{Bracket Types and Cements}

The characteristics of the bracket bases and cements used in this study are summarized in

Tables 2 and 3 (Page 39).

Table 2. BRACKET TYPES AND THEIR BASES

\begin{tabular}{|l|l|l|l|}
\hline $\begin{array}{l}\text { BRACKET } \\
\text { MATERIAL }\end{array}$ & \multicolumn{1}{|c|}{ NAME } & \multicolumn{1}{|c|}{ MANUFACTURER } & \multicolumn{1}{|c|}{ BASE TRATMENT } \\
\hline Metal & Speed $^{4}$ & $\begin{array}{l}\text { Strite Industries, } \\
\text { Cambridge, Ontario. }\end{array}$ & $\begin{array}{l}\text { 60 gauge, microetched } \\
\text { foil-mesh base }\end{array}$ \\
\cline { 2 - 4 } & Time $^{3}$ & $\begin{array}{l}\text { American Orthodontics, } \\
\text { Wisconsin. }\end{array}$ & $\begin{array}{l}\text { Machined, integral, } \\
\text { microetched base with } \\
\text { mechanical undercuts }\end{array}$ \\
\hline \multirow{2}{*}{ Ceramic } & Clarity & $\begin{array}{l}\text { Unitek Corporation, } \\
\text { Monrovia, California. }\end{array}$ & $\begin{array}{l}\text { Polycrystalline ceramic } \\
\text { bracket using crystal-like } \\
\text { particles fused to the } \\
\text { bracket base for } \\
\text { mechanical retention }\end{array}$ \\
& $\begin{array}{l}\text { Transcend } \\
\text { 6000 }\end{array}$ & $\begin{array}{l}\text { Unitek Corporation, } \\
\text { Monrovia, California. }\end{array}$ & $\begin{array}{l}\text { Polycrystalline ceramic } \\
\text { bracket using crystal-like } \\
\text { particles fused to the } \\
\text { bracket base for } \\
\text { mechanical retention }\end{array}$ \\
\hline
\end{tabular}


Table 3. PROPERTIES AND CHARACTERISTICS OF THE BONDING CEMENTS

\begin{tabular}{|c|c|c|c|}
\hline Brand Name & Fuji Ortho LC ${ }^{5}$ & Transbond $^{7}$ & Panavia21 $^{6}$ \\
\hline Manufacturer & $\begin{array}{l}\text { GC America Inc } \\
\text { Chicago, IL. }\end{array}$ & $\begin{array}{l}\text { Unitek Corporation, } \\
\text { Monrovia, } \\
\text { California. }\end{array}$ & $\begin{array}{l}\text { Kuraray Co., Ltd } \\
\text { Osaka, Japan. }\end{array}$ \\
\hline Description & $\begin{array}{l}\text { Hybrid glass } \\
\text { ionomer cement } \\
\text { reinforced with } \\
\text { composite }\end{array}$ & $\begin{array}{l}80 \% \text { filled by } \\
\text { weight, microfilled } \\
\text { composite resin } \\
\text { cement }\end{array}$ & $\begin{array}{l}77 \% \text { filled by weight, } \\
\text { low viscosity } \\
\text { composite resin, } \\
\text { containing } 10- \\
\text { Methacryloyloxydecyl } \\
\text { dihydrogen phosphate }\end{array}$ \\
\hline Preparation & $\begin{array}{l}\text { Powder mixed into } \\
\text { liquid manually }\end{array}$ & One paste system & $\begin{array}{l}\text { Catalyst and Universal } \\
\text { paste incorporated into } \\
\text { a dispensing unit and } \\
\text { mixed manually }\end{array}$ \\
\hline $\begin{array}{l}\text { Polymerization } \\
\text { Method }\end{array}$ & Photo-polymerized & Photo-polymerized & $\begin{array}{l}\text { Auto-polymerized in } \\
\text { the absence of oxygen }\end{array}$ \\
\hline $\begin{array}{l}\text { Fluoride } \\
\text { Release }\end{array}$ & Yes & No & No \\
\hline $\begin{array}{l}\text { Enamel } \\
\text { Treatment }\end{array}$ & $\begin{array}{l}\text { Either conditioned } \\
\text { with } 10 \% \\
\text { Polyacrylic Acid } \\
\text { solution or no } \\
\text { conditioning at all }\end{array}$ & $\begin{array}{l}\text { Etching with } 37 \% \\
\text { Orthophosphoric } \\
\text { Acid followed by } \\
\text { application of } \\
\text { Heliobond (photo- } \\
\text { polymerising } \\
\text { adhesive primer) } \\
\end{array}$ & $\begin{array}{l}\text { ED Primer (Liquid A } \\
\text { \& B) } \\
\text { Applied to enamel } \\
\text { surface, left for } 60 \\
\text { seconds and air dried to } \\
\text { leave a glossy surface }\end{array}$ \\
\hline Dry Field & No & Yes & Yes \\
\hline
\end{tabular}




\section{Bracket Base Bonding Surface Area}

The bonding surface-areas of the bracket bases used in the study were measured using a morphometric program ${ }^{8}$ (Digitek Image processing system, series 100, Digitek co., Brooklyn N.Y., 1988). Random samples of 10 brackets from each type were taken, and their mean base surface areas were recorded using the method described by MacColl (1995). The mean bonding surface area for each type of bracket was calculated and the results are recorded in Table 4.

Table 4. BRACKET BONDING BASE AREA IN MM'

\begin{tabular}{|l|c|}
\hline Bracket Type ( $\mathbf{N}=10$ Brackets) & Area in $\mathbf{m m}^{2}+/-$ Standard Deviation \\
\hline Speed & $7.35+/-0.18$ \\
\hline Time & $13.00+/-0.17$ \\
\hline Clarity & $11.66+/-0.22$ \\
\hline Transcend 6000 & $10.53+/-0.19$ \\
\hline
\end{tabular}

\section{Preparation of the Teeth}

Bovine mandibular incisor teeth were collected from an abattoir. Following the removal of their roots on a band saw, the crowns were stored in distilled water in a freezer at $-20^{\circ} \mathrm{C}$ until used. Prior to embedding in acrylic, the teeth were thawed in warm water and the pulp tissues were extirpated with a dental explorer. The empty pulp chamber of each tooth was rinsed with distilled water and then packed with moist cotton pledgets. Plastic cylinders (25 $\mathrm{mm}$ in diameter $\times 20 \mathrm{~mm}$ in depth) were filled to one third of their depth with autopolymerizing acrylic resin and allowed to polymerize. The teeth were then placed on top of the hardened acrylic and secured with a piece of plasticene ${ }^{9}$ so that the labial surfaces faced upwards and were parallel to the horizontal plane. Auto-polymerizing acrylic resin was then poured around the tooth, leaving the labial surface exposed. Once the acrylic was fully polymerized, the embedded specimens were removed from their plastic cylinders. Immediately prior to bracket cementation, a flat surface was created on labial surface of each tooth by grinding it with water irrigated \#180 and \#600 grit Silicon Carbide (SiC) paper $^{10}$. Care was taken not to expose the underlying dentin. The teeth were then rinsed for 30 seconds in running distilled water and dried using a hair dryer. The brackets were bonded in groups of 12 in accordance with the protocols shown in Table 5 (Page 41). 
Table 5. Experimental Protocols

\begin{tabular}{|c|c|c|c|c|c|}
\hline \multirow{2}{*}{$\begin{array}{l}\text { Bracket } \\
\text { Type }\end{array}$} & \multirow[t]{2}{*}{ Adhesive } & \multirow[t]{2}{*}{ Enamel Treatment } & \multicolumn{2}{|c|}{ Storage } & \multirow[t]{2}{*}{$\mathbf{N}$} \\
\hline & & & 1 day & 180 days & \\
\hline Speed & $\begin{array}{l}\text { Transbond } \\
\text { Panavia21 } \\
\text { Fuji Ortho LC } \\
\text { Fuji Ortho LC }\end{array}$ & $\begin{array}{l}\text { Conditioned, } 37 \% \text { phosphoric acid, dry } \\
\text { Self-etching Acidic Primer, dry } \\
\text { Conditioned, } 10 \% \text { Polyacrylic Acid, wet } \\
\text { Not-conditioned, wet }\end{array}$ & $\begin{array}{l}\text { Yes } \\
\text { Yes } \\
\text { Yes } \\
\text { Yes }\end{array}$ & $\begin{array}{l}\text { Yes } \\
\text { Yes } \\
\text { Yes } \\
\text { No }\end{array}$ & $\begin{array}{l}12 \\
12 \\
12 \\
12\end{array}$ \\
\hline Time & $\begin{array}{l}\text { Transbond } \\
\text { Panavia21 } \\
\text { Fuji Ortho LC } \\
\text { Fuji Ortho LC }\end{array}$ & $\begin{array}{l}\text { Conditioned, } 37 \% \text { phosphoric acid, dry } \\
\text { Self-etching Acidic Primer, dry } \\
\text { Conditioned, } 10 \% \text { Polyacrylic Acid, wet } \\
\text { Not-conditioned, wet }\end{array}$ & $\begin{array}{l}\text { Yes } \\
\text { Yes } \\
\text { Yes } \\
\text { Yes }\end{array}$ & $\begin{array}{l}\text { Yes } \\
\text { Yes } \\
\text { Yes } \\
\text { No }\end{array}$ & $\begin{array}{l}12 \\
12 \\
12 \\
12\end{array}$ \\
\hline $\begin{array}{l}\text { Transcend } \\
6000\end{array}$ & $\begin{array}{l}\text { Transbond } \\
\text { Panavia21 } \\
\text { Fuji Ortho LC } \\
\text { Fuji Ortho LC }\end{array}$ & $\begin{array}{l}\text { Conditioned, } 37 \% \text { phosphoric acid, dry } \\
\text { Self-etching Acidic Primer, dry } \\
\text { Conditioned, } 10 \% \text { Polyacrylic Acid, wet } \\
\text { Not-conditioned, wet }\end{array}$ & $\begin{array}{l}\text { Yes } \\
\text { Yes } \\
\text { Yes } \\
\text { Yes }\end{array}$ & $\begin{array}{l}\text { Yes } \\
\text { Yes } \\
\text { Yes } \\
\text { No }\end{array}$ & $\begin{array}{l}12 \\
12 \\
12 \\
12\end{array}$ \\
\hline Clarity & $\begin{array}{l}\text { Transbond } \\
\text { Panavia21 } \\
\text { Fuji Ortho LC } \\
\text { Fuji Ortho LC }\end{array}$ & $\begin{array}{l}\text { Conditioned, } 37 \% \text { phosphoric acid, dry } \\
\text { Self-etching Acidic Primer, dry } \\
\text { Conditioned, } 10 \% \text { Polyacrylic Acid, wet } \\
\text { Not-conditioned, wet }\end{array}$ & $\begin{array}{l}\text { Yes } \\
\text { Yes } \\
\text { Yes } \\
\text { Yes }\end{array}$ & $\begin{array}{l}\text { Yes } \\
\text { Yes } \\
\text { Yes } \\
\text { No }\end{array}$ & $\begin{array}{l}12 \\
12 \\
12 \\
12\end{array}$ \\
\hline
\end{tabular}




\section{Bonding Protocol}

All of the specimens were bonded in the following manner regardless of the type of cement used. The clean flattened dry enamel surface was prepared as described previously. The cement was prepared according to the manufacturer's instructions and was then applied to the bracket base with a plastic instrument. The bracket was then seated with its slot parallel with the incisal edge and held in position using the guide pin of a semi-adjustable Hanau articulator $^{13}$ (Teledyne Hanau, Buffalo, NY, 14225). A stone (dental stone) cylinder weighing 610 grams attached to the upper member of the articulator was used to provide a standardized and constant force during bracket placement (MacColl, 1995). This enabled the investigator to use both hands to carefully remove the excess extruded bonding agent with a sharp explorer and, at the same time produce a consistently thin layer of cement (MacColl, 1995). The cements were then light cured, using a visible-light-curing unit ${ }^{14}$, for 20 seconds on the mesial and distal sides of the bracket base, for a total of $\mathbf{4 0}$ seconds (Transbond ${ }^{7}$ and Fuji Ortho $\mathrm{LC}^{5}$ ) or allowed to auto-polymerize (Panavia2 $1^{6}$ ). The output of the light unit was measured after polymerizing every five specimens with a curing radiometer ${ }^{15}$ to insure a constant light intensity value of at least $380 \mathrm{~mW} / \mathrm{cm}^{2}$ (Rueggeberg et al., 1994).

\section{Bonding Protocol for Transbond ${ }^{7}$}

The clean flattened dry enamel surface was conditioned with $37 \%$ orthoposphoric acid ${ }^{11}$ for 20 seconds and then rinsed with distilled water for 30 seconds and hot air dried with a hair drier $^{16}$. Heliobond ${ }^{12}$ bonding agent was brushed in a thin layer onto the conditioned enamel surface and light-cured for 10 seconds. The Transbond ${ }^{\top}$ cement was applied to the bracket base with a plastic instrument, and the bracket placed near the center of the labial surface of the tooth. The cement was then photo-polymerized, using a visible-light-curing unit ${ }^{14}$, for 20 seconds on the mesial and distal sides of the bracket, for a total of 40 seconds.

\section{Bonding Protocol for Fuji Ortho LC}

Conditioned (etched) and not-conditioned (not etched) bonding protocols were followed for Fuji Ortho $\mathrm{LC}^{\mathrm{S}}$ cement. When the enamel surface was conditioned, it was conditioned by brushing $10 \%$ polyacrylic acid ${ }^{17}$ onto the labial surface. The conditioner was left undisturbed for 20 seconds and then rinsed thoroughly with distilled water for 30 seconds. In both 
groups the enamel surface was not dried but was moistened with distilled water just before bonding. The Fuji Ortho $\mathrm{LC}^{5}$ cement was mixed following manufacturer's guidelines. One level spoonful of powder and two drops of liquid were dispensed on to a mixing pad. The powder was incorporated into liquid in two equal portions. The first portion was mixed into the liquid for 10 seconds. The second part was then added and mixed for another 10-15 seconds. The total mixing time did not exceed 20-25 seconds. The brackets were bonded to enamel surfaces as described in the bonding protocol section. Each mix was used to bond two brackets only. The cement was then polymerized for $\mathbf{4 0}$ second as described above.

\section{Bonding Protocol for Panavia21 ${ }^{6}$}

Prior to bracket bonding, the ED primer ${ }^{18}$ (self-etching acidic primer) was mixed and applied to the dry enamel surface and hot air dried after 60 seconds. Panavia2 $1^{6}$ was dispensed using the two-paste delivery system and mixed in accordance with the manufacturer's instructions and then applied to bracket bonding surface. The bracket was then placed on the prepared tooth surface, excess cement removed and left to set undisturbed for 15 minutes before storage in distilled water.

\section{Storage}

Upon completion of bonding and curing procedures, each specimen was placed in a small self-capping plastic container in distilled water. A few crystals of thymol were added to prevent bacterial growth (Rueggeberg, 1991). The specimens were then placed in a humidor at $37^{\circ} \mathrm{C}$ for 24 -hours or 180 -days. The water and thymol solution was changed once a week for the specimens that were stored for 180-days.

\section{Shear Bond Strength Testing}

The specimens were shear tested to failure using an Instron Universal Testing Machine ${ }^{19}$ (model 4301, Instron Corporation, 100 Royal Street, Canton, Mass, 02021). Each specimen was clamped in a holding ring so that the bracket base was parallel to the direction of the force. The sharpened chisel blade suspended from the moving arm of the testing machine was placed at the bracket enamel interface just short of contact in an incisogingival direction. Using a one a Kilo-Newton $(\mathrm{KN})$ compression cell and a crosshead speed of 0.5 
$\mathrm{mm} / \mathrm{minute}$ the bracket bases were shear tested to failure. The maximum force was recorded in Newtons (N) and converted to megapascals (MPa). Diagrams of the testing apparatus can be viewed in Mahal (2000) thesis, pages 77 and 78.

\section{Nature of Bond Failure}

The Adhesive Remnant Index (ARI) (Table 1- Page 34) was used to quantify the amount of cement left on the tooth following debonding of the bracket. (Artun and Bergland, 1984). The ARI scores were also used to define the sites of bond failure between the enamel, the cement, and the bracket base. The ARI consists of a 4-point scale of 0-3: a score of 0 indicates no cement left on the tooth, 1 indicates less than half of the cement left on the tooth, 2 indicates more than half of the cement left on the tooth, and 3 indicates all of the cement left on the tooth including a distinct impression of the bracket base.

The adhesive remnant index score was reported for each debonded specimen by examining the bracket bases and enamel surfaces under a light microscope $\mathrm{e}^{20}$ at a magnification of $\times 35$.

\section{Inter and Intra-Operator Error Study on ARI}

To test for possible intra-operator error in assigning the ARI scores, 12 debonded specimens were selected and viewed under the light microscope ${ }^{20}$ by the principal investigator on two separate occasions. A second examiner also viewed the same specimens in order to assess for inter-operator reliability.

\section{Scanning Electron Microscopy (SEM)}

A cylindrical sample of each cement used in this study was prepared by following the manufacturer guidelines for mixing as described previously. The cylinders of each cement were obtained by placing the cement in a hole punched into a Polyvinyl Chloride (PVC) clear mouthguard material with thickness and diameter of $3 \mathrm{~mm}$ and photo-polymerized (Transbond ${ }^{7}$, Fuji Ortho $L C^{5}$ ) or auto-polymerized (Panavia2 $1^{6}$ ). The bases of these cylindrical cement samples were then polished with \#180 grit, \#600 grit and finally \#4000 grit $\mathrm{SiC}$ paper $^{10}$ on a water irrigated grinding wheel. These specimens and one of each bracket type with the bases facing up were mounted on an aluminum SEM stub. All the 
specimens were then sputter coated with $3 \mathrm{~nm}$ of platinum in a Polaron E5100 SEM coating unit and viewed with a Hitachi-S-2500 SEM ${ }^{21}$ at an operating voltage of $10 \mathrm{kV}$ (Sharma, 1999).

Scanning electron microscopy views of each specimen were obtained at $\times 25, \times 100$ and $x 1000$ magnifications. The average cement particle size was determined using the $\times 1000$ magnification SEM images of each cement. The SEM micrograph of each bracket base was matched with the three cements at the same magnification. Visual analysis was made to investigate compatibility of each bracket base surface characteristic with the cement particle size.

\section{Statistical Analysis}

Descriptive statistics including the mean and standard deviation values of the SBS and ARI scores were calculated for each of the groups tested.

A one-way analysis of variance (ANOVA) test was used to determine the effect of bracket base design on the mean SBS. The one-way analysis of variance was also used to study the effect of the cement material on the mean ARI scores and the mean SBS. A two-way analysis of variance was used to determine the effect of long-term storage on mean SBS. This two-way analysis of variance was also used to study the effects of bracket base design, cement type and the interaction between these two variables on the mean SBS. Duncan's multiple range test was used to group different cement materials with respect to SBS and ARI scores. This test was also used to group different bracket base designs with respect to the mean SBS. Multiple pair wise comparisons of the least square means were carried out to compare the mean SBS recorded with various bracket base-cement combinations.

A linear regression analysis was used to determine if there was any association between the SBS and ARI scores. Fischer's Exact test was used to determine the effect of long-term storage on the distribution of ARI scores. An un-weighted Kappa statistics was used to highlight inter-operator and intra-operator differences when assigning ARI scores. The statistical significance for all the tests was established at a 5\% level. The SAS system was used for all the above analysis of the data (SAS 6.23, 1996, Cary, NC). 


\section{RESULTS}

\section{CLINICAL ACCEPTABILITY OF VARIOUS BRACKET BASE-CEMENT COMBINATIONS}

The criteria for clinical acceptability was defined as ARI scores of 2-3 and mean shear bond strengths (SBS) in the range of $8 \pm 3 \mathrm{MPa}$. The mean ARI scores and the mean SBS values of all the bracket base-cement combinations tested after 1-day and 180-days of storage are summarized in Figure 1 (Page 48) and Figure 2 (Page 49).

\section{i) 1-Day Storage Group}

The mean SBS and the corresponding ARI scores for the various bracket base-cement combinations tested after 1-day of storage are summarized in Table 6 (Page 50). Of all the 16 bracket base-cement combinations tested after 1-day of storage 69\% (11 out of 16) were clinically acceptable. Therefore, only $31 \%$ (5 out of 16) performed in unacceptable fashion. These were Speed-Panavia21, Time-Panavia21, Time-Fuji Ortho LC (not-conditioned), Transcend-Fuji Ortho LC (not-conditioned), Clarity-Fuji Ortho LC (not-conditioned) combinations. These combinations are highlighted in Table 6 (Page 50). These bracket basecement combinations were deemed clinically unacceptable because of low SBS (less than 5 $\mathrm{MPa}$ ) and/or low ARI scores (less than 2).

\section{ii) 180-Days Storage Group}

The mean SBS and the corresponding ARI scores for the various bracket base-cement combinations tested after 180-days of storage are summarized in Table 7 (Page 51). Of all the 12 combinations tested after 180-days of storage $83 \%$ (10 out of 12 ) were clinically acceptable and $17 \%$ (2 out of 12) of these combinations debonded in an unfavorable fashion. These were Speed-Panavia21 and Time-Panavia21 combinations that produced acceptable bond strengths but low ARI scores with most of the cement remaining on the bracket base after debonding. All of the bracket base-cement combinations tested after 180-days of storage produced clinically acceptable SBS. This increase in the clinical acceptability of the various bracket base-cement combinations after 180-days of storage was because of the omission of the combinations (brackets bonded to not-conditioned enamel using Fuji Ortho LC) that produced poor SBS and/or ARI scores in the 1-day storage group. 
If the effects of time on clinical acceptability are compared between the same bracket basecement combinations at the two storage times (i.e. eliminating the Fuji Ortho LC notconditioned protocol), then it becomes evident that the storage time had no significant effect on the clinical acceptability. At both storage times, Speed-Panavia21 and Time-Panavia21 combinations performed poorly. Therefore, the clinical acceptability at time 1-day and 180days of storage was $83 \%$ ( 10 out of 12 bracket base-cement combinations). 


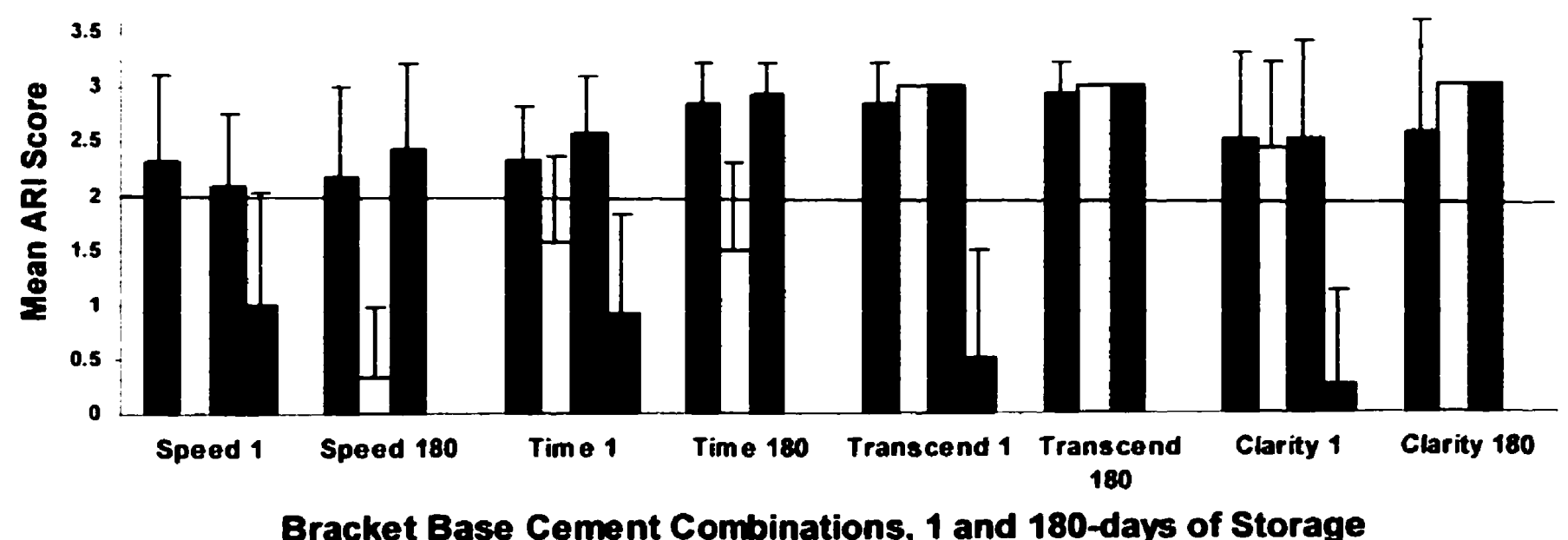

Transbond $\square$ Panavia $\square$ Fuji (Cond) E Fuji (No Cond)

Figure 1: Mean ARI Scores for Various Bracket Base-Cement Combinations Tested After 1 and 180-Days of Storage

The horizontal black line indicates the acceptable ARI scores; in this study it was considered to be $\geq 2$ 
Hillithilititit

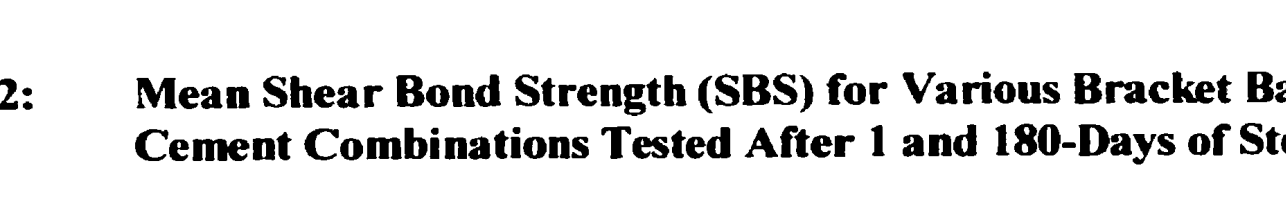


Table 6: Shear Bond Strength (SBS) and Adhesive Remnant Index (ARI) Scores Percent Distribution (1-Day of Storage)

\begin{tabular}{|c|c|c|c|c|c|c|c|c|c|c|c|c|c|c|c|c|c|}
\hline 霝 & $\begin{array}{l}\text { BONDING } \\
\text { PROTOCOL }\end{array}$ & \multicolumn{4}{|c|}{$\begin{array}{l}\text { Transbond } \\
\text { Conditioned with } \\
\text { 37\% Phosphnric } \\
\text { acid, dry }\end{array}$} & \multicolumn{4}{|c|}{$\begin{array}{l}\text { Panavia21" } \\
\text { Self-etching Acidic } \\
\text { Primer, dry }\end{array}$} & \multicolumn{4}{|c|}{$\begin{array}{l}\text { Fujl Ortho LC } \\
\text { Conditioned with } \\
10 \% \text { Polyacrylic } \\
\text { acid, wet }\end{array}$} & \multicolumn{4}{|c|}{$\begin{array}{l}\text { Fuji Ortho LC } \\
\text { Not- conditioned, } \\
\text { wet }\end{array}$} \\
\hline \multirow{3}{*}{$\begin{array}{l}\text { 蛅 } \\
\text { 号 }\end{array}$} & SBS in MPa & \multicolumn{4}{|c|}{$11.69 \pm 2.3$} & \multicolumn{4}{|c|}{$9.34 \pm 1.20$} & \multicolumn{4}{|c|}{$11.79 \pm 2.2$} & \multicolumn{4}{|c|}{$6.47 \pm 3.13$} \\
\hline & ARI Score & $\mathbf{0}$ & $\mathbf{I}$ & 2 & 3 & 0 & 1 & 2 & 3 & 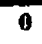 & I & 2 & 3 & $\mathbf{0}$ & & 2 & 3 \\
\hline & Distribution \% & 0 & 17 & 33 & 50 & $\frac{\pi}{1}$ & $\overline{0}$ & 0 & 0 & 0 & 25 & 50 & 25 & 42 & 0 & 58 & 0 \\
\hline \multirow{3}{*}{$\underline{E}$} & SAS in MPa & \multicolumn{4}{|c|}{$12.75 \pm 2.66$} & \multicolumn{4}{|c|}{$11.07 \pm 1.26$} & \multicolumn{4}{|c|}{$8.5 \pm 1.69$} & \multicolumn{4}{|c|}{$48 \pm 23$} \\
\hline & ARI Score & $\mathbf{0}$ & $\mathbf{I}$ & 2 & 3 & 0 & 1 & 2 & 3 & 0 & $\mathbf{I}$ & 2 & 3 & O & $\mathbf{I}$ & 2 & 3 \\
\hline & Distribution \% & 0 & $\mathbf{0}$ & 67 & 33 & 0 & 8 & 25 & 17 & 0 & $\mathbf{0}$ & 42 & 58 & 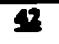 & 23 & 33 & 0 \\
\hline \multirow{3}{*}{ 를 } & SDS in MPa & \multicolumn{4}{|c|}{$15.33 \pm 4.86$} & \multicolumn{4}{|c|}{$10.63 \pm 1.57$} & \multicolumn{4}{|c|}{$10.30 \pm 2.18$} & \multicolumn{4}{|c|}{$7.77 \pm 1.73$} \\
\hline & ARI Score & $\mathbf{0}$ & $\mathbf{I}$ & 2 & 3 & o & 1 & 2 & 3 & 0 & 1 & 2 & 3 & - & 1 & 2 & 3 \\
\hline & Distribution \% & 0 & $\mathbf{0}$ & 17 & 83 & 0 & $\mathbf{0}$ & 0 & 10 & 0 & $\mathbf{0}$ & 0 & 10 & $\pi$ & 8 & 8 & 8 \\
\hline \multirow{3}{*}{ 를 } & SRS in MPa & \multicolumn{4}{|c|}{$13.38 \pm 4.27$} & \multicolumn{4}{|c|}{$9.47 \pm 3.06$} & \multicolumn{4}{|c|}{$9.91 \pm 2.90$} & \multicolumn{4}{|c|}{$5.09 \pm 1.9$} \\
\hline & ARI Score & 0 & 1 & 2 & $\mathbf{3}$ & 0 & 1 & 2 & 3 & $\mathbf{0}$ & 1 & 2 & 3 & o & 1 & 2 & 3 \\
\hline & Distribution \% & 0 & 17 & 17 & 64 & 0 & 17 & 25 & 58 & 8 & 0 & 25 & 67 & $\boldsymbol{2}$ & $\mathbf{0}$ & $\mathbf{0}$ & 8 \\
\hline
\end{tabular}

The bracket base-cement combinations with ARI and/or SBS that are highlighted and underlined are clinically unacceptable. 
Table 7. Shear Bond Strength (SBS) and Adhesive Remnant Index (ARI) Scores Percent Distribution (180-Days of Storage)

\begin{tabular}{|c|c|c|c|c|c|c|c|c|c|c|c|c|c|}
\hline Ex & $\begin{array}{l}\text { BONDING } \\
\text { PROTOCOL }\end{array}$ & \multicolumn{4}{|c|}{$\begin{array}{l}\text { Transbond" } \\
\text { Conditioned with } 37 \% \\
\text { phosphoric acid, dry }\end{array}$} & \multicolumn{4}{|c|}{$\begin{array}{l}\text { Panavia21 } \\
\text { Self-etching acidic } \\
\text { primer, dry }\end{array}$} & \multicolumn{4}{|c|}{$\begin{array}{l}\text { Fuji Ortho LC } \\
\text { Conditioned with } 10 \% \\
\text { Polyacrylic acid, wet }\end{array}$} \\
\hline \multirow{3}{*}{ 弯 } & SRS in MPa & \multicolumn{4}{|c|}{$14.05 \pm 2.84$} & \multicolumn{4}{|c|}{$8.77 \pm 1.32$} & \multicolumn{4}{|c|}{$12.69 \pm 2.13$} \\
\hline & ARI Score & 0 & I & 2 & 3 & o & 1 & 2 & 3 & 0 & 1 & 2 & 3 \\
\hline & Distribution \% & $\mathbf{0}$ & 25 & 33 & 42 & $\boldsymbol{T}$ & II & 8 & 0 & 0 & 17 & 25 & 58 \\
\hline \multirow{3}{*}{ 兽 } & SES in MPa & \multicolumn{4}{|c|}{$12.1 \pm 3.23$} & \multicolumn{4}{|c|}{$9.61 \pm 13.5$} & \multicolumn{4}{|c|}{$8.44 \pm 1.69$} \\
\hline & ARI Score & 0 & $\mathbf{I}$ & 2 & 3 & 0 & 1 & 2 & 3 & 0 & 1 & 2 & 3 \\
\hline & Distribution \% & 0 & $\mathbf{0}$ & 17 & 83 & 0 & $\mathbf{S}$ & 17 & 17 & 0 & 0 & 8 & 92 \\
\hline \multirow{3}{*}{ "气 } & SaS in MPa & \multicolumn{4}{|c|}{$14.76 \pm 4.56$} & \multicolumn{4}{|c|}{$8.32 \pm 1.5$} & \multicolumn{4}{|c|}{$9.84 \pm 2.07$} \\
\hline & ARI Score & $\mathbf{0}$ & 1 & 2 & 3 & 0 & 1 & 2 & 3 & $\mathbf{0}$ & 1 & 2 & 3 \\
\hline & Distribution \% & $\mathbf{0}$ & 0 & 8 & 92 & 0 & 0 & 0 & 100 & 0 & $\mathbf{0}$ & 0 & 100 \\
\hline \multirow{3}{*}{ 롤 } & SaS in MPa & \multicolumn{4}{|c|}{$11.86 \pm 3.35$} & \multicolumn{4}{|c|}{$8.64 \pm 2.46$} & \multicolumn{4}{|c|}{$10.13 \pm 2.58$} \\
\hline & ARI Score & $\mathbf{0}$ & 1 & 2 & 3 & 0 & 1 & 2 & 3 & 0 & 1 & 2 & 3 \\
\hline & & 8 & 8 & 0 & 84 & 0 & 0 & 0 & 100 & 0 & 0 & 0 & 100 \\
\hline
\end{tabular}

The bracket base-cement combinations with ARI scores that are highlighted and underlined are clinically unacceptable. 


\section{COMPARISON OF MEAN SBS (1-DAY OF STORAGE)}

\section{i) Comparison of Mean SBS of Various Bracket Base-Cement Combinations (1- Day of Storage)}

The mean SBS after 1-day of storage of 16 different bracket base-cement combinations were compared using a two-way ANOVA test. In this test the mean SBS was the dependent variable and the bracket base types, cement materials, and the bracket base-cement combinations were the sources of variation. $P$ values of less than 0.05 were recorded for brackets $(p=0.0071)$ and cements $(p=0.0001)$, which demonstrate that these variables had a significant effect on mean SBS (Table 8). The interaction between the bracket base and the cement material was also significant at $(p=0.0103)$. The result of this test showed that there was a statistically significant difference between the SBS of the 16 various bracket basecement combinations tested after 1 -day of storage $(p=0.0103)$. (Table 8)

Table 8: Two-way Analysis of Variance for Mean SBS of 4 Bracket Bases and 4 Cements/Bonding Protocols (1-day of storage)

\begin{tabular}{|c|c|c|c|c|}
\hline Source of variation & df & Sum of squares & Mean squares & $\mathbf{P}$ \\
\hline $\begin{array}{l}\text { Bracket } \\
\text { Cement } \\
\text { Bracket }{ }^{\star} \text { Cement }\end{array}$ & $\begin{array}{l}3 \\
3 \\
9\end{array}$ & $\begin{array}{l}87.07 \\
1274.664 \\
157.102\end{array}$ & $\begin{array}{l}29.034 \\
424.888 \\
17.455\end{array}$ & $\begin{array}{l}\text { 0.0071 } \\
0.0001 \\
0.0103\end{array}$ \\
\hline
\end{tabular}

Multiple pairwise comparisons of least square means were carried out to perform pairwise comparisons between the mean SBS of 16 bracket base-cement combinations tested after 1day of storage. The results of this test are summarized in Table 14 (Page 57) and the combinations that are significantly different from each other are denoted with * symbol. 


\section{ii) Comparison of Mean SBS of Various Bracket Bases (1-Day of Storage)}

A Duncan's Multiple Range test was carried out to identify the bracket bases that resulted in different mean SBS. To perform this test, the mean SBS of each bracket base type was calculated by pooling and averaging the mean SBS resulting from all the cement types for each bracket base type. Then the mean SBS of each bracket base type was compared with the other three bracket base types. The mean SBS with the same letter as indicated by the Duncan's grouping are not statistically significantly different. (Table 9) Therefore, there was no significant difference between the bond strengths recorded using Speed, Time or Clarity bracket bases. Of all the bracket bases tested after 1-day of storage, Transcend bracket bases had the highest mean SBS and performed significantly differently than the other three bracket bases. Speed, Time and Clarity bracket bases had mean SBS that were statistically similar.

Table 9: Duncan's Grouping of the Mean SBS for Different Bracket Base Types (1-day of storage, holding the cements constant)

\begin{tabular}{|llcc|}
\hline Bracket Type & N & Mean SBS (MPa) & Duncan grouping \\
\hline Speed $^{4}$ & 48 & 9.83 & B \\
Time $^{3}$ & 48 & 9.27 & B \\
Transcend $^{2}$ & 48 & 11.0 & A \\
Clarity' $^{1}$ & 48 & 9.46 & B \\
\hline
\end{tabular}

Groups with different letters are significantly different from each other.

\section{iii) Comparison of Mean SBS of Various Cements (1-Day of Storage)}

A one-way ANOVA test was carried out in order to compare the mean SBS resulting from different cements and independent of bracket base design. The results showed that there was a statistically significant difference between the mean SBS recorded for each cement material after 1-day of storage $(p=0.0001)$. Then a Duncan's Multiple Range test was performed to identify the cement materials that had significantly different mean SBS (Table 10- Page 54). 


\section{Table 10: Duncan's Grouping of Mean SBS for Different Cement Material (1-day of storage, holding the brackets constant)}

\begin{tabular}{|llcc|}
\hline Cement Material & N & Mean SBS (MPa) & Duncan grouping \\
\hline Transbond & 48 & 13.29 & A \\
Panavia & 48 & 10.13 & B \\
Fuji Ortho (Cond) & 48 & 10.13 & B \\
Fuji Ortho (no Cond) & 48 & 6.03 & C \\
\hline
\end{tabular}

Groups with different letters are significantly different from each other.

The mean SBS with the same letter as indicated by the Duncan's grouping are not statistically significantly different (Table 10). The results showed that there was no significant difference between the bond strengths recorded using Panavia2 1 or Fuji Ortho LC (conditioned). Of all the cements tested after 1-day of storage, Transbond cement produced the highest mean SBS and performed significantly differently than the other three cements. Fuji Ortho LC (not-conditioned) resulted in the lowest mean SBS and performed significantly differently than all the other cements.

\section{COMPARISON OF MEAN SBS (180-DAYS OF STORAGE)}

\section{i) Comparison of Mean SBS of Various Bracket Base-Cement Combinations (180-Days of Storage)}

The mean SBS after 180-days of storage of 12 different bracket base cement combinations were compared using a 2-way ANOVA test. In this test the mean SBS was the dependent variable and the bracket base types, cement materials, and the bracket base-cement combinations were the sources of variation. $P$ values of less than 0.05 were recorded for brackets $(p=0.0157)$ and cements $(p=0.0001)$ thus these variables had a significant effect on the mean SBS (Table 11- Page 55). The interaction between the bracket base and the cement material was also significant at $(\mathrm{p}=0.0071)$. The result of this test showed that there was a statistically significant difference between the mean SBS of the 12 various bracket basecement combinations tested after 180 -days of storage $(p=0.0071)$. (Table 11 - Page 55) 
Table 11: Two-way Analysis of Variance for SBS of 4 Bracket Bases and 4 Cements/Bonding Protocols (180-days of storage)

\begin{tabular}{|lcccc|}
\hline Source of variation & df & Sum of squares & Mean squares & P \\
\hline Bracket & 3 & 72.47 & 24.16 & $\mathbf{0 . 0 1 6}$ \\
Cement & 2 & 473.13 & 236.56 & $\mathbf{0 . 0 0 0 1}$ \\
Bracket*Cement & 6 & 125.48 & 20.91 & $\mathbf{0 . 0 0 7 1}$ \\
& & & & \\
\hline
\end{tabular}

$\mathbf{P}<0.05$ denotes statistical significance

$\mathrm{df}=$ degrees of freedom

Multiple pairwise comparisons of least square means were carried out to perform pair wise comparisons between the mean SBS of 12 bracket base-cement combinations tested after 180-days of storage. The results of this test are summarized in Table 15 (Page 58) and the combinations that are significantly different from each other are denoted with ${ }^{\star *}$ symbol.

A Duncan's Multiple Range Test was carried out to identify the brackets that resulted in different mean SBS. To perform this test, the mean SBS of each bracket base type was calculated by pooling and averaging the mean SBS resulting from all the cement types for each bracket base types. Then the mean SBS of each bracket base type was compared with the other three bracket base types. The mean SBS with the same letters as indicated by the Duncan's grouping are not significantly different (Table 12). Therefore, there was no significant difference between the bond strengths recorded using Transcend, Time or Clarity bracket bases. Speed bracket bases yielded the highest mean SBS which was significantly greater than the mean SBS resulting from using the other three bracket base types: Time, Transcend and Clarity.

Table 12: Duncan's Grouping of the Mean SBS for Different Bracket Base Types (180-days of storage, holding the cements constant)

\begin{tabular}{|llcc|}
\hline Bracket tested & N & Mean SBS (MPa) & Duncan grouping \\
\hline Speed & 36 & 11.84 & A \\
Time & 36 & 10.05 & B \\
Transcend & 36 & 10.97 & AB \\
Clarity & 36 & 10.2 & B \\
\hline
\end{tabular}

Groups with different letters are significantly different from each other. 


\section{ii) Comparison of Mean SBS of Various Cements (180-Day of Storage)}

A one-way ANOVA test was carried out in order to compare mean SBS resulting from different cements and independent of bracket base design. The results of this test showed that there was a statistically significant difference between the mean SBS produced by each cement material after 180-days of storage $(p=0.0001)$. Then a Duncan's Multiple Range test was used to identify the cements that were different with respect to recorded mean SBS. (Table 13)

\section{Table 13: Duncan's Grouping of the Mean SBS for Different Cement Material} (180-days of storage, holding the brackets constant)

\begin{tabular}{|lccc|}
\hline Cement Material & N & Mean SBS (MPa) & Duncan grouping \\
\hline Transbond & 48 & 13.19 & A \\
Panavia & 48 & 10.27 & B \\
Fuji Ortho (Cond) & 48 & 8.83 & C \\
\hline
\end{tabular}

Groups with different letters are significantly different from each other.

The mean SBS with different letters as indicated by the Duncan's grouping are significantly different (Table 13). Therefore, there were significant differences between the bond strengths recorded using Transbond, Panavia21 and Fuji Ortho LC (conditioned) cements. Of all the cements tested after 180-days of storage, Transbond cement produced the highest mean SBS and performed significantly differently than the other two cements. Fuji Ortho LC (conditioned) cement resulted in the lowest mean SBS and performed significantly differently than Transbond and Panavia 21 cements. 
Table 14: The Pairwise Comparison of Mean SBS of Various Bracket Base-Cement Combinations (1-Day of Storage)

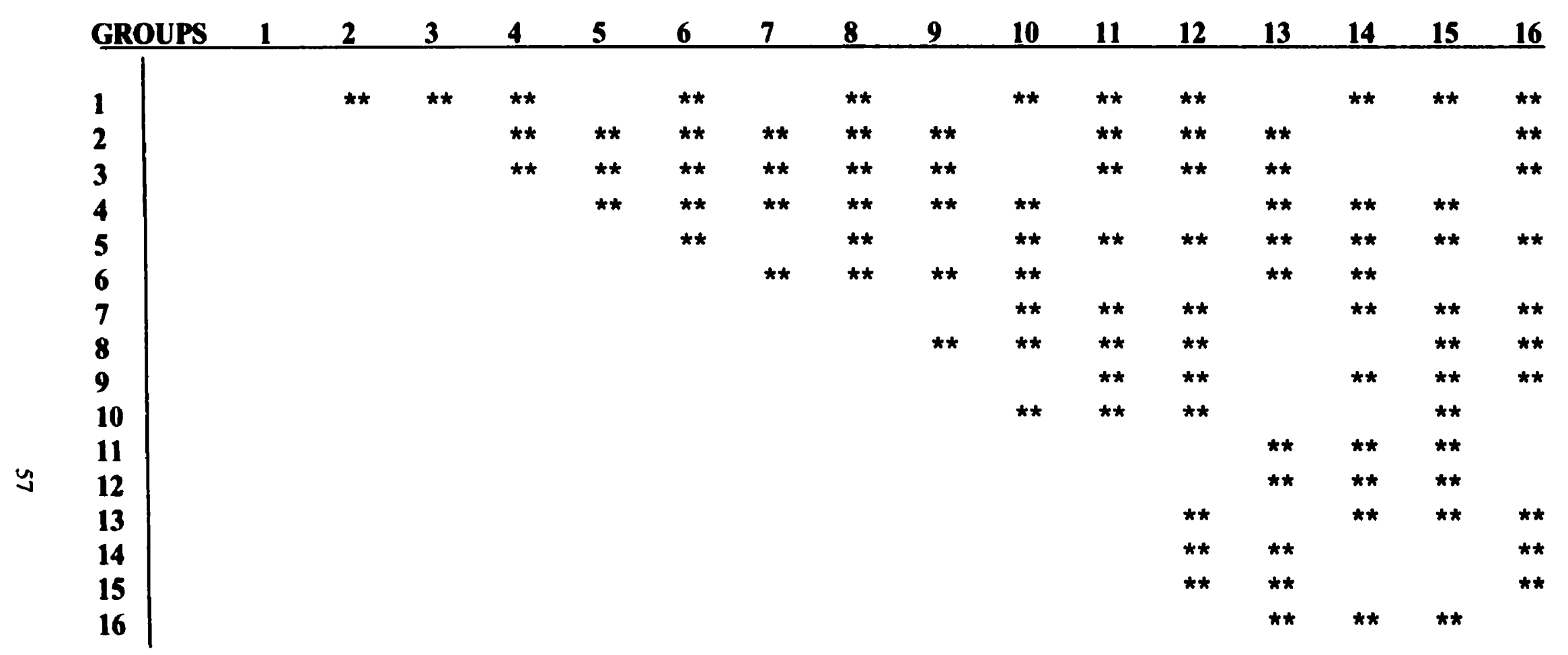

** denotes significance at $p<0.05$

Leqend

1 Clarity-Fuji Ortho LC (not-conditioned)

9 Time-Fuji Ortho LC (not-conditioned)

2 Clarity-Fuji Ortho LC (conditioned)

3 Clarity-Pannvia21

4 Clarity-Transbond

5 Speed-Fuji Ortho LC (not-conditioned)

6 Speed-Fuji Ortho LC (conditioned)

7 Speed-Panavia21

8 Speed-Transbond

10 Time-Fuji Ortho LC (conditioned)

11 Time-Panavia21

12 Time-Transbond

13 Transcend-Fuji Ortho LC (not-conditioned)

14 Transcend-Fuji Ortho LC (conditioned)

15 Transcend-Panavia21

16 Transcend-Transbond 
Table 15: The Pairwise Comparison of Mean SBS of Various Bracket Base-Cement Combinations (180-Days of Storage)

\begin{tabular}{|c|c|c|c|c|c|c|c|c|c|c|c|c|}
\hline GROUPS & 1 & 2 & 3 & 4 & 5 & 6 & 7 & 8 & 9 & 10 & 11 & 12 \\
\hline 1 & & & & $\star \star$ & $* *$ & & & & $\star \star$ & & $\star *$ & $\star \star$ \\
\hline 2 & & & ** & & ** & & & ** & $\star \star$ & & & $\star *$ \\
\hline 3 & & & & $\star \star \star$ & $\star \star *$ & $\star \star$ & ** & & & $\star \star$ & $\star \star \star$ & \\
\hline 4 & & & & & $\star \star *$ & & & $\star \star *$ & $\star \star *$ & & & ** \\
\hline 5 & & & & & & $\star \star$ & ** & ** & ** & $\star *$ & $\star *$ & $* *$ \\
\hline 6 & & & & & & & & & ** & & & $\star *$ \\
\hline 7 & & & & & & & & & $\star \star *$ & & & ** \\
\hline 8 & & & & & & & & & $\star \star *$ & & $\star \star$ & $\star *$ \\
\hline 9 & & & & & & & & & & ** & $\star *$ & \\
\hline 10 & & & & & & & & & & & & $\star \star$ \\
\hline 11 & & & & & & & & & & & & $\star \star$ \\
\hline
\end{tabular}

$\star \star$ denotes Significance at $\mathbf{P}<0.05$

Lepend

1 Clarity-Fuji Ortho LC (conditioned)

7 Time-Fuji Ortho LC (conditioned)

2 Clarity-Panavia21

3 Clarity-Transbond

4 Speed-Fuji Ortho LC (conditioned)

5 Speed-Panavia21

6 Speed-Transbond

8 Time-Panavia21

9 Time-Transbond

10 Transcend-Fuji Ortho LC (conditioned)

11 Transcend-Panavia21

12 Transcend-Transbond 


\section{THE EFFECT OF LONG-TERM STORAGE ON MEAN SBS OF VARIOUS BRACKET BASE-CEMENT COMBINATIONS}

In summary, the storage time of 180-days had a statistically significant effect on the mean SBS of $25 \%$ ( 3 out of 12) of the bracket base-cement combinations tested. $8 \%$ ( 1 out of 12) of the samples showed statistically significant increases in SBS while $17 \%$ (2 out of 12) demonstrated reductions in SBS. However, these changes were not clinically significant.

\section{i) Speed Brackets Bonded with Different Cements}

Long-term storage significantly increased the mean SBS of Speed brackets bonded with Transbond cement ( $p=0.037)$. Long-term storage, however, had no statistically significant effect on the mean SBS of Speed brackets bonded with Panavia21 $(p=0.269)$ or Fuji Ortho LC (conditioned) cements $(\mathrm{p}=0.32)$. The mean SBS of Speed brackets bonded with Panavia21 decreased slightly after 180-days of storage. The mean SBS of Speed brackets bonded with Fuji Ortho LC (conditioned) cement increased slightly when tested after 180days of storage. (Table 16- Page 63, Figure 3)

Figure 3: Comparison of Mean SBS after 1 and 180-Days of Storage for Speed Brackets Bonded with Different Cements

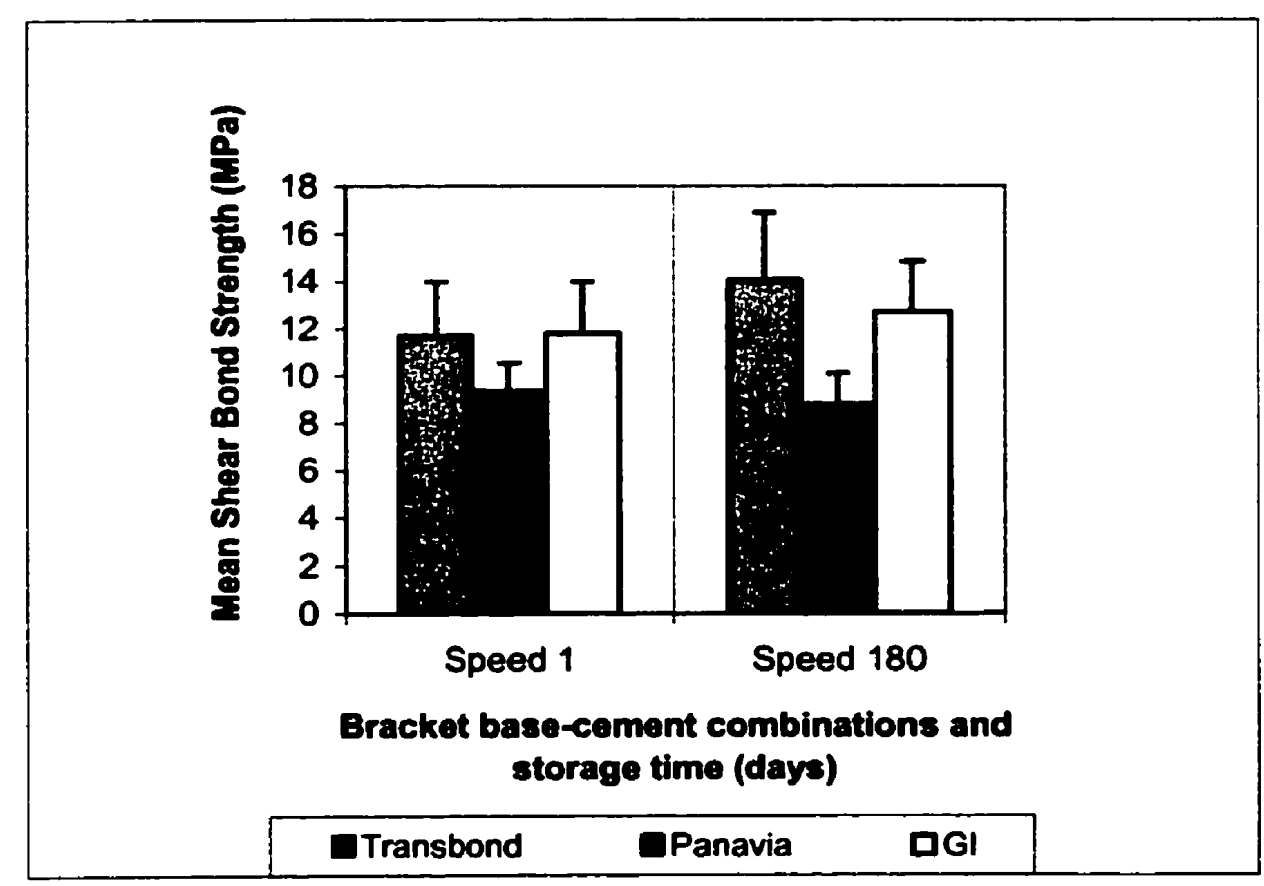

Legend for Figures 3-6: Panavia= Panavia2l GI= Fuji Ortho LC (conditioned) 


\section{ii) Time Brackets Bonded with Different Cements}

Long-term storage significantly decreased the mean SBS of Time brackets bonded with Panavia21 cement $(p=0.012)$. However, long-term storage had no significant effect on the mean SBS of Time brackets bonded with Transbond $(p=0.596)$ or Fuji Ortho LC (conditioned) cement $(\mathrm{p}=\mathbf{0 . 9 2 8 )}$. The mean SBS of Time brackets bonded with Transbond cement decreased slightly after 180-days of storage and the mean SBS of those bonded with Fuji Ortho LC (conditioned) cement was basically unchanged. (Table 16- Page 63, Figure 4)

Figure 4: Comparison of Mean SBS after 1 and 180-Days of Storage for Time Brackets Bonded with Different Cements

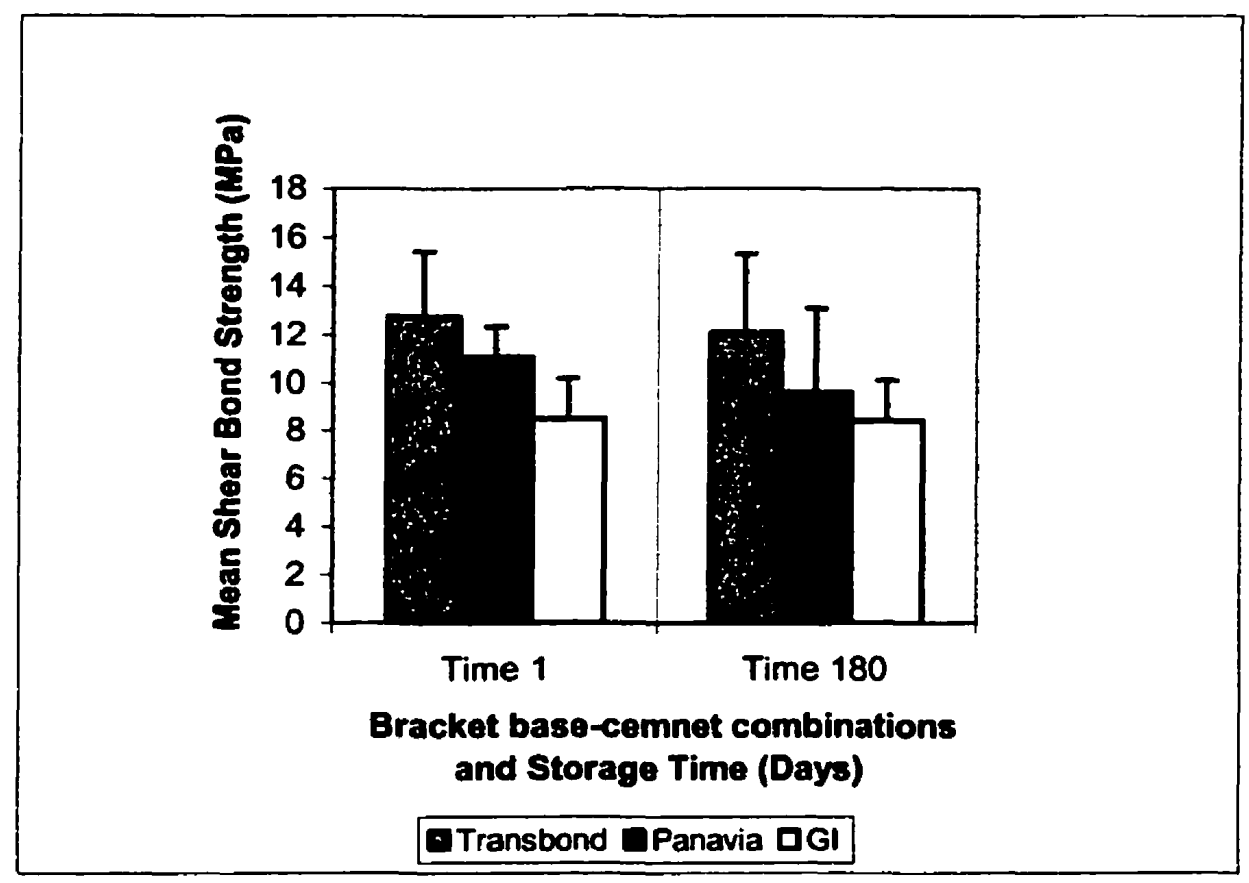




\section{iii) Transcend Brackets Bonded with Different Cements.}

Long-term storage significantly decreased the mean SBS of Transcend brackets bonded with Panavia2I cement $(p=0.001)$. Long-term storage, however, had no significant effect on the mean SBS of Transcend brackets bonded with Transbond $(p=0.768)$ or Fuji Ortho LC (conditioned) cements $(p=0.597)$. The mean SBS of Transcend brackets bonded with Transbond or Fuji Ortho LC (conditioned) was reduced slightly after 180-days of storage. These changes were not statistically or clinically significant. (Table 16- Page 63, Figure 5)

Figure 5: Comparison of Mean SBS after 1 and 180-Days of Storage for Transcend Brackets Bonded with Different Cements

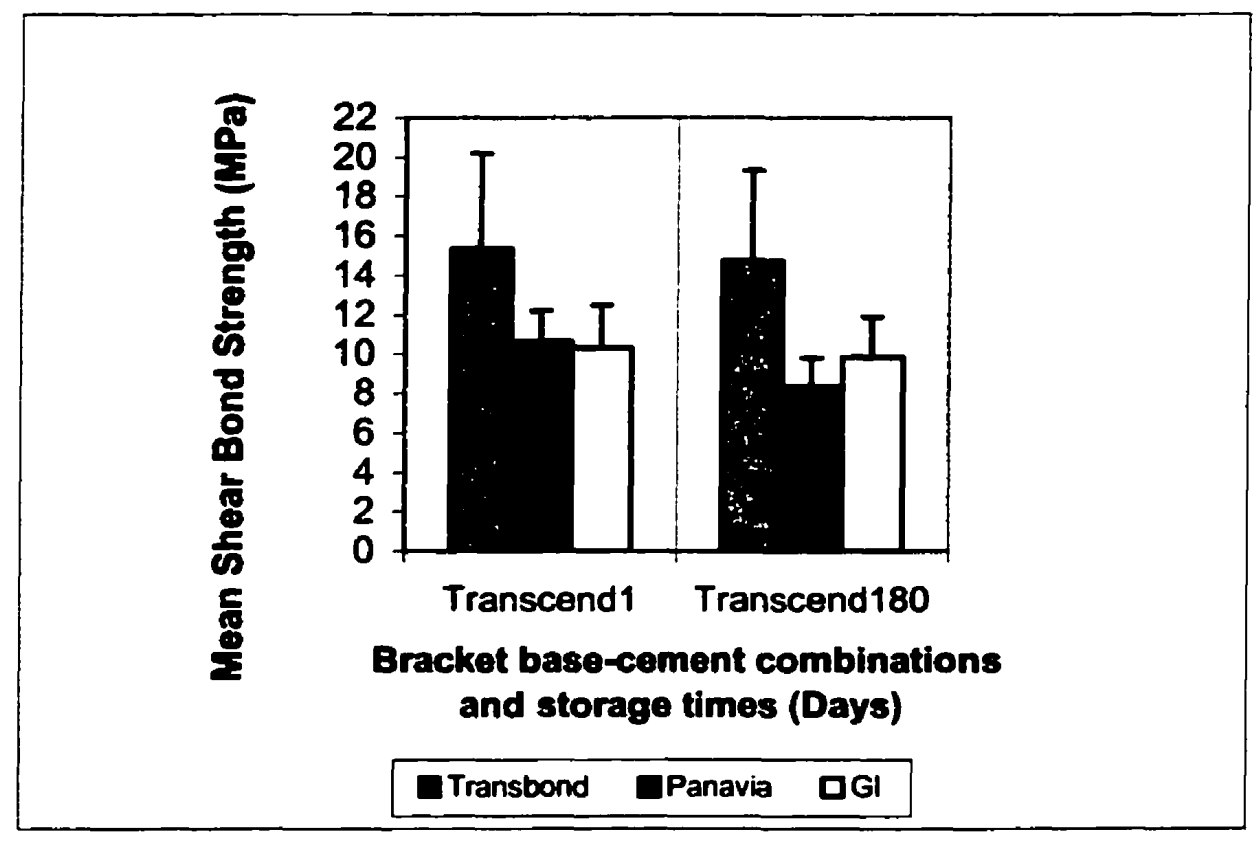


iv) Clarity Brackets Bonded with Different Cements.

Long-term storage had no significant effect on the mean SBS of Clarity brackets bonded with the three different cements. The mean SBS of Clarity brackets bonded with Transbond $(p=0.343)$ or Panavia21 $(p=0.473)$ decreased slightly after 180 days. Clarity brackets bonded with Fuji Ortho LC (conditioned) showed a slight increase in mean SBS after 180-days of storage $(p=0.844)$. (Table 16- Page 63, Figure 6)

Figure 6: Comparison of Mean SBS after 1 and 180-Days of Storage for Clarity Brackets Bonded with Different Cements

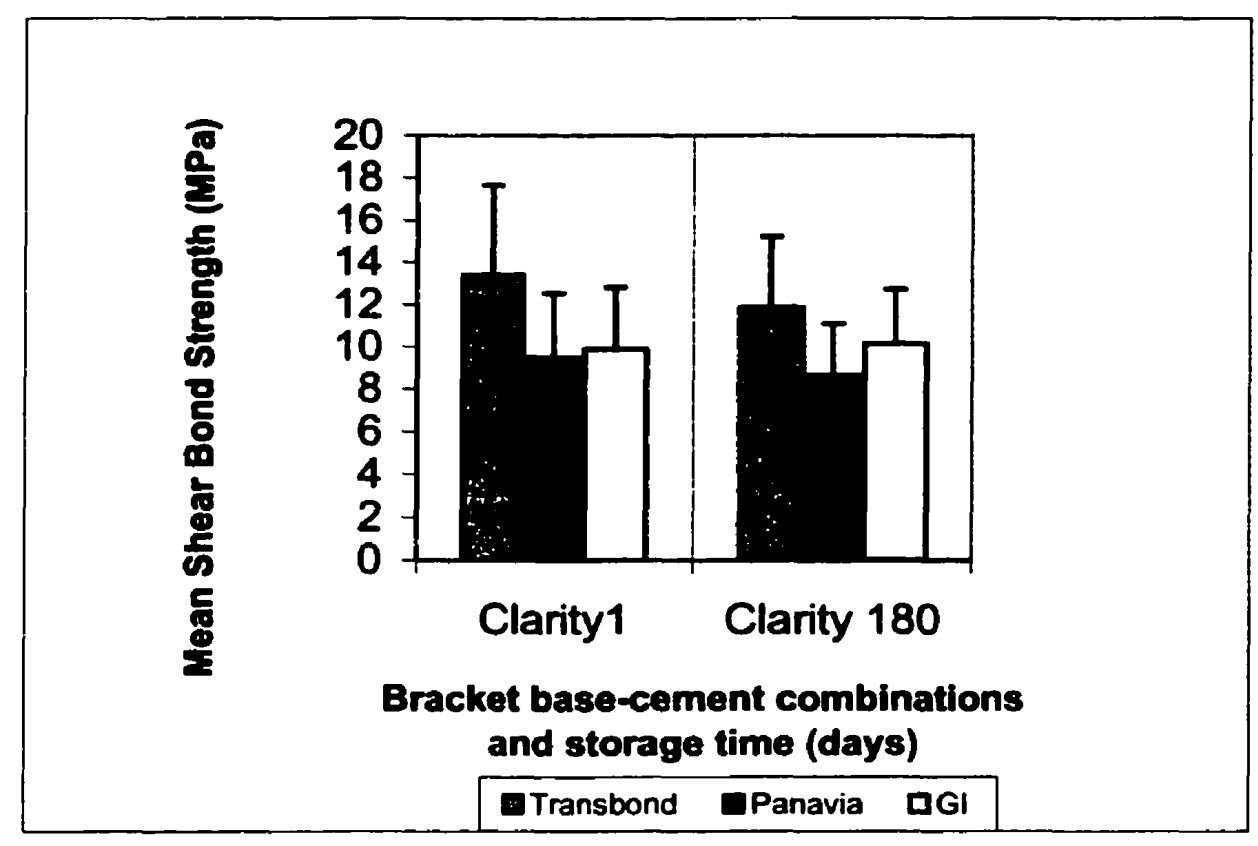


Table 16: Comparison of the Mean Shear Bond Strengths (SBS)

(1 and 180-Days of Storage)

\begin{tabular}{|c|c|c|c|c|c|c|c|c|c|}
\hline \multirow{2}{*}{$\begin{array}{l}\text { Bonding } \\
\text { Protocol } \\
\text { Bracket } \\
\text { Type }\end{array}$} & \multicolumn{3}{|c|}{$\begin{array}{l}\text { Transbond' } \\
\text { Conditioned with } 37 \% \\
\text { Phosphoric Acid, dry }\end{array}$} & \multicolumn{3}{|c|}{$\begin{array}{l}\text { Panavia21 } \\
\text { Self-etching Acidic } \\
\text { primer, dry }\end{array}$} & \multicolumn{3}{|c|}{$\begin{array}{l}\text { Fuji Ortho LC } \\
\text { Conditioned with } 10 \% \\
\text { Polyacrylic Acid, wet }\end{array}$} \\
\hline & $\begin{array}{l}\text { 1-day } \\
\text { Stornge }\end{array}$ & $\begin{array}{l}\text { 180-days } \\
\text { Storage }\end{array}$ & P value & $\begin{array}{l}\text { 1-day } \\
\text { Storage }\end{array}$ & $\begin{array}{l}\text { 180-days } \\
\text { Storage }\end{array}$ & $\begin{array}{l}P \\
\text { value }\end{array}$ & $\begin{array}{l}\text { 1-day } \\
\text { Storage }\end{array}$ & $\begin{array}{l}\text { 180-days } \\
\text { Storage }\end{array}$ & $\begin{array}{l}P \\
\text { value }\end{array}$ \\
\hline Speed & $11.69+23$ & $14.05+284$ & 0.037 & $9.34 \pm 1.20$ & $8.77 \pm 1.32$ & 0.269 & $11.79 \pm 2.2$ & $12.69 \pm 2.13$ & 0.32 \\
\hline Time $^{3}$ & $12.75 \pm 2.66$ & $12.1 \pm 3.23$ & 0.596 & $11.07 \pm 1.26$ & $9.61 \pm 13.5$ & 0.012 & $8.5 \pm 1.69$ & $8.44 \pm 1.69$ & 0.928 \\
\hline Transcend $^{2}$ & $15.33 \pm 4.86$ & $14.76 \pm 4.56$ & 0.768 & $10.63 \pm 1.57$ & $8.32 \pm 1.5$ & 0.001 & $10.30 \pm 2.18$ & $9.84 \pm 2.07$ & 0.597 \\
\hline Clarity' & $13.38 \pm 4.27$ & $11.86 \pm 3.35$ & 0.343 & $9.47 \pm 3.06$ & $8.64 \pm 2.46$ & 0.473 & $9.91 \pm 2.90$ & $10.13 \pm 2.58$ & 0.844 \\
\hline
\end{tabular}

$\mathbf{P}<0.05$ denotes statistical significance

Long-term storage had a statistically significant effect on the shear bond strength of bracket base-cement combinations that are highlighted. 


\section{ARI SCORES DISTRIBUTION AFTER 1-DAY OF STORAGE}

The mean ARI scores for various bracket base-cement combinations tested after 1-day of storage are summarized in Table 17.

Table 17: Mean ARI Scores for Various Bracket Base-Cement Combinations (1-day of storage)

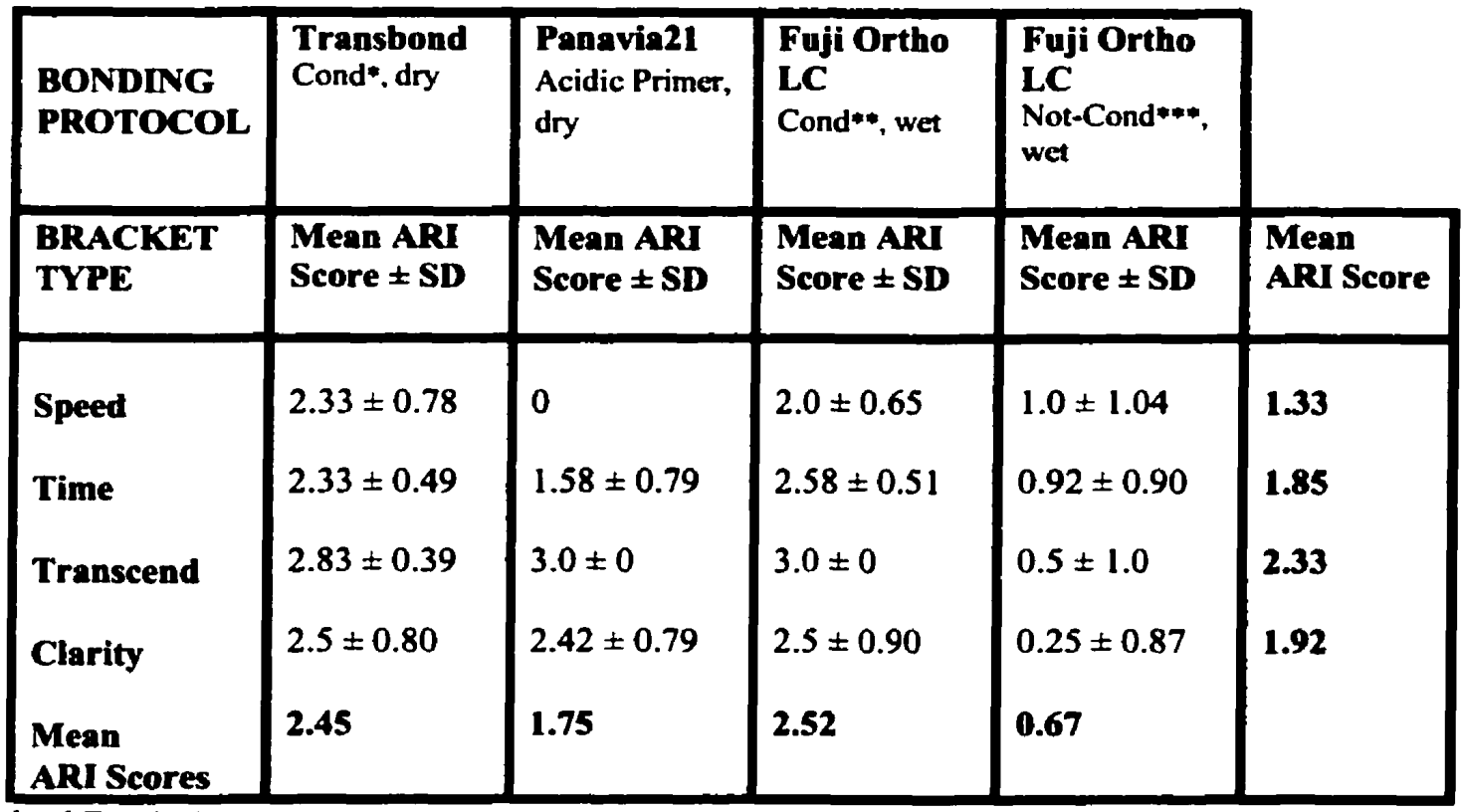

$\mathrm{SD}=$ Standard Deviation

Sample size $=12$

Storage time $=1$ day

*Conditioned with 37\% Phosphoric Acid

* Conditioned with 10\% Polyacrylic Acid

*** Enamel surface not conditioned

Bracket bases bonded with Transbond and Fuji Ortho LC (conditioned) cements demonstrated the most favourable ARI scores when debonded after 1-day of storage.

\section{Comparison of ARI Scores Associated with Various Cements (1-Day of Storage)}

A one-way ANOVA test was carried out in order to compare the ARI scores resulting from different cements and independent of bracket base design. The results of this test showed that there was a statistically significant difference between the ARI scores produced by 
different cements after 1-day of storage $(p=0.0001)$. A Duncan's Multiple Range test was then used to identify the cements that were different with respect to ARI scores. The results of this test are summarized in Table 18.

Table 18: Duncan's Grouping of the ARI Scores Associated with Different Cement Materials (1-day of storage, holding the brackets constant)

\begin{tabular}{|llcc|}
\hline Cement Material & N & Mean ARI Score & Duncan grouping \\
\hline Transbond & 48 & 2.45 & A \\
Fuji Ortho (Cond) & 48 & 2.52 & A \\
Panavia 21 & 48 & 1.75 & B \\
Fuji Ortho (no Cond) & 48 & 0.67 & C \\
\hline
\end{tabular}

Groups with different letters are significantly different from each other.

The mean ARI Scores with different letters as indicated by the Duncan's grouping are significantly different (Table 18). There were no significant differences between the mean ARI scores recorded using Transbond, Fuji Ortho LC (conditioned) cements. This indicates that these two cements debonded in a similar fashion leaving more than half of the cement on the enamel. Panavia21 cement produced significantly different mean ARI scores than other cements. After debonding brackets bonded with Panavia2I less than half of the cement was left on the tooth. Of all the cements tested after 1-day of storage, Fuji Ortho LC (not conditioned) produced significantly different ARI scores than the other cements. Fuji Ortho LC (not conditioned) produced the poorest ARI scores and less than half of the cement was left on enamel after debonding brackets bonded with Fuji Ortho LC (not conditioned).

\section{THE MEAN ARI SCORES FOR VARIOUS BRACKET BASE-CEMENT COMBINATIONS (1-DAY OF STORAGE)}

\section{i) The Mean ARI Scores for Brackets Bonded with Transbond (1-day of storage)}

The mean ARI scores for brackets bonded with Transbond cement ranged from 2.33 to 2.83 . These scores indicate that failures for all the bracket base types bonded with Transbond cement occurred at the bracket base-cement interface with more than half of the cement remaining on the tooth. These results indicate that all bracket base types cemented with 
Transbond resulted in clinically acceptable (ARI $\geq 2$ ) bond failure location, which was identified at bracket base-cement interface with most of the cement remaining on the enamel. (Tables 6 and 17- Pages 50 and 64)

\section{ii) The Mean ARI Scores for Brackets Bonded with Panavia21 (1-day of storage)}

The mean ARI scores for bracket bases bonded with Panavia2l cement range from 0 to 3 . Speed and Transcend brackets bonded with Panavia21 produced the lowest and the highest ARI scores respectively. These scores indicate that failures for Transcend $(A R I=3)$ and Clarity brackets ( $A R I=2.42)$ occurred at bracket base-cement interface with more than half of the cement remaining on the tooth. The Speed brackets bonded with Panavia 21 cement produced the poorest ARI scores ( $\mathrm{ARI}=0$ ) with all the cement remaining on the bracket base. Time brackets bonded with Panavia21 also resulted in poor ARI scores $(A R I=1.58)$ with most of the cement remaining on the bracket base after debonding. (Tables 6 and 17- Pages 50 and 64)

iii) The Mean ARI Scores for Brackets Bonded with Fuji Ortho LC (conditioned) (1-day of storage)

The mean ARI scores for brackets bonded with Fuji Ortho LC (conditioned) cement ranged from 2 to 3 with Speed and Transcend brackets producing the lowest and the highest ARI scores respectively. These scores indicate that failures for Transcend (ARI=3), Time $(A R I=2.58)$, Clarity ( $A R I=2.42)$ and Speed brackets $(A R I=2.0)$ were all favourable and occurred at the bracket base-cement interface with greater than half of the cement remaining on the tooth. (Tables 6 and 17- Pages 50 and 64)

\section{iv) The Mean ARI Scores for Brackets Bonded with Fuji Ortho LC (not-conditioned) (1-day of storage)}

The mean ARI scores for brackets bonded with Fuji Ortho LC (not-conditioned) cement ranged from 0.5-0.92. Therefore, the mean ARI scores for all the bracket types bonded with Fuji Ortho LC (not-conditioned) were all unfavourable and most of the cement was left on the bracket base after debonding. (Tables 6 and 17- Pages 50 and 64) 


\section{ARI SCORES DISTRIBUTION (180-DAYS OF STORAGE)}

The mean ARI scores for various bracket base-cement combinations tested after 180-days of storage are summarized in Table 19.

\section{Table 19: Mean ARI Scores for Various Bracket Base-Cement Combinations (180-days of storage)}

\begin{tabular}{|l|l|l|l|l|}
\hline $\begin{array}{l}\text { BONDING } \\
\text { PROTOCOL }\end{array}$ & $\begin{array}{l}\text { Transbond } \\
\text { Cond*, dry }\end{array}$ & $\begin{array}{l}\text { Panavia 21 } \\
\text { Acidic Primer, } \\
\text { dry }\end{array}$ & $\begin{array}{l}\text { Fuji Ortho } \\
\text { LC } \\
\text { Cond } * *, \text { wet }\end{array}$ & \\
\hline $\begin{array}{l}\text { BRACKET } \\
\text { TYPE }\end{array}$ & $\begin{array}{l}\text { Mean ARI } \\
\text { Score } \pm \text { SD }\end{array}$ & $\begin{array}{l}\text { Mean ARI } \\
\text { Score } \pm \text { SD }\end{array}$ & $\begin{array}{l}\text { Mean ARI } \\
\text { Score } \pm \text { SD }\end{array}$ & $\begin{array}{l}\text { Mean ARI } \\
\text { Scores }\end{array}$ \\
\hline Speed & $2.17 \pm 0.83$ & $0.33 \pm 0.65$ & $2.42 \pm 0.79$ & 1.63 \\
Time & $2.83 \pm 0.39$ & $1.5 \pm 0.80$ & $2.92 \pm 0.29$ & 2.42 \\
Transcend & $2.92 \pm 0.29$ & $3.0 \pm 0$ & $3 \pm 0$ & 2.97 \\
Clarity & $2.58 \pm 1.0$ & $3.0 \pm 0$ & $3 \pm 0$ & 2.86 \\
Mean ARI & 2.62 & 1.95 & 2.8 & \\
\hline Score & & & \\
\hline
\end{tabular}

$\mathrm{SD}=$ Standard Deviation

Sample size $=12$

Storage time $=1$ day

.Conditioned with 37\% Phosphoric Acid

*Conditioned with 10\% Polyacrylic Acid

Bracket bases bonded with Transbond and Fuji Ortho LC (conditioned) cement demonstrated the most favourable ARI scores when debonded after 180-days of storage.

\section{Comparison of ARI Scores Associated with Various Cements (180-Days of Storage)}

A one-way ANOVA test was carried out in order to compare the ARI scores resulting from different cements and independent of bracket base design. The results of this test showed 
that there was a statistically significant difference between the ARI scores produced by different cements after 180-days of storage $(p=0.0001)$. Then a Duncan's Multiple Range test was performed to identify the cements that were different with respect to ARI scores. The results of this test are summarized in Table 20.

Table 20: Duncan's Grouping of the ARI Scores Associated with Different

Cement Materials (180-days of storage, holding the brackets constant)

\begin{tabular}{|llcc|}
\hline Cement Material & N & Mean ARI Score & Duncan grouping \\
\hline Transbond & 48 & 2.63 & A \\
Fuji Ortho (Cond) & 48 & 2.83 & A \\
Panavia 21 & 48 & 1.96 & B \\
\hline
\end{tabular}

Groups with different letters are significantly different from each other.

The mean ARI scores with different letters (Table 20) as indicated by the Duncan's grouping are significantly different. The results show that there were no significant differences between the mean ARI scores recorded using Transbond, Fuji Ortho LC (conditioned) cements. This indicates that these two cements debonded in a similar fashion leaving more than half of the cement on the tooth. Panavia21 cement produced significantly different mean ARI scores and less than half of the cement was left on the tooth after debonding.

\section{THE MEAN ARI SCORES FOR VARIOUS BRACKET BASE-CEMENT COMBINATIONS (180-DAYS OF STORAGE)}

\section{i) The Mean ARI Scores for Brackets Bonded with Transbond (180-days of storage)}

The mean ARI scores for brackets bonded with Transbond cement ranged from 2.16 to 2.92 . These scores indicate that failures for all the bracket base types bonded with Transbond cement occurred at the bracket base-cement interface with more than half of the cement remaining on the tooth. These results indicate that all bracket base types cemented with Transbond resulted in clinically acceptable $(A R I \geq 2)$ bond failure locations that were 
identified at bracket base-cement interface with most of the cement remaining on the tooth surface. (Tables 7 and 19- Pages 51 and 67)

\section{ii) The Mean ARI Scores for Brackets Bonded with Panavia21 (180-days of storage)}

The mean ARI scores for brackets bonded with Panavia2l cement ranged from 0.3 to 3 with Speed brackets producing the lowest ARI score and Transcend and Clarity brackets bonded with Panavia2l producing the highest ARI scores. The mean ARI score for the Time brackets bonded with Panavia21 was 1.5 indicating that most of the cement was left on the bracket base after debonding. (Tables 7 and 19- Pages 51 and 67)

\section{iii) The Mean ARI Scores for Brackets Bonded with Fuji Ortho LC (conditioned) (180-days of storage)}

The mean ARI scores for brackets bonded with Fuji Ortho LC (conditioned) cement ranged from 2.4 to 3. Bond failures for all bracket base types bonded with Fuji Ortho LC (conditioned) were all favourable and occurred at the bracket base-cement interface with more than half (Speed and Time brackets) or all of the cement (Transcend and Clarity brackets) remaining on the tooth after debonding. (Tables 7 and 19- Pages 51 and 67) 


\section{THE EFFECT OF LONG-TERM STORAGE ON THE FREQUENCY OF ARI SCORES DISTRIBUTION}

Table 21 (Page 73) includes a summary of ARI scores distribution for each bracket basecement combination. The results of the two-tail Fischer's Exact test comparing the ARI scores distribution after 1 and 180-days of storage are also recorded in this table.

\section{i) Speed Brackets Bonded with Transbond}

A two-tail Fischer's Exact test showed no significant differences between the ARI scores at times of 1 and 180-days of storage when Speed brackets were bonded with the Transbond cement. The ARI scores for both the 1 and 180-day storage groups ranged from 1 to 3 with more than half of the scores being 2 and 3. (Table 21- Page 73)

\section{ii) Speed Brackets Bonded with Panavia21}

A two-tail Fischer's Exact test showed a significant difference between the ARI scores at times of 1 and 180-days of storage when Speed brackets were bonded with Panavia21 cement. The ARI scores after 1-day of storage were all 0 , however, after 180-days of storage a significant shift was noted in the distribution of the ARI scores $(p<0.001)$. After 180-days of storage the ARI scores ranged from 0 to 2 with $76 \%$ of the scores being 0 . (Table 21 , Page 73)

iii) Speed Brackets Bonded with Fuji Ortho LC (conditioned)

A two-tail Fischer's Exact test showed a significant difference between the ARI scores at times 1 and 180-days of storage when Speed brackets were bonded with Fuji Ortho LC (conditioned) cement $(p<0.001)$. The ARI scores after 1 -day of storage ranged from 1 to 3 with the majority of scores being 2 . After 180-days of storage there was a significant change in the ARI scores distribution so that the range was 1 to 3 with the majority of scores being 3. (Table 21, Page 73) 


\section{iv) Time Brackets Bonded with Transbond}

A two-tail Fischer's Exact test showed a significant difference between the ARI scores at times of 1 and 180-days of storage when Time brackets were bonded with Transbond cement $(p<0.001)$. The ARI scores ranged from 2 to 3 after both the 1 and 180-days storage periods. For the 1-day storage group $67 \%$ of the ARI scores were 2 and for the 180-days storage group $83 \%$ of the ARI scores were 3. (Table 21 - Page 73)

v) Time Brackets Bonded with Panavia21

A two-tail Fischer's Exact test showed a significant difference between the ARI scores at time 1 and 180-days of storage when Time brackets were bonded with Panavia2l cement. The ARI scores for both storage times ranged from 1 to 3. (Table 21-Page 73)

vi) Time Brackets Bonded with Fuji Ortho LC (conditioned)

A two-tail Fischer's Exact test showed a significant difference between the ARI scores at times of 1 and 180-days of storage when Time brackets were bonded with Fuji Ortho LC (conditioned) cement $(\mathrm{p}<0.001)$. The ARI scores for both storage times ranged from 2 to 3 , however $92 \%$ of the ARI scores were 3 for the 180-days storage group as compared to $58 \%$ ARI scores of 3 for the 1-day storage group. (Table 21-Page 73)

\section{vii) Transcend Brackets Bonded with Transbond}

A two-tail Fischer's Exact test showed no significant differences between the ARI scores at times of 1 and 180-days of storage when Transcend brackets were bonded with Transbond cement. The ARI scores for both storage times ranged from 2 to 3, with more than $50 \%$ of the scores being 3 for both of the storage times. (Table 21-Page 73)

\section{viii) Transcend Brackets Bonded with Panavia21}

A two-tail Fischer's Exact test was not performed for this combination since the ARI scores for both of the storage times were all 3. (Table 21 - Page 73) 


\section{ix) Transcend Brackets Bonded with Fuji Ortho LC (conditioned)}

The two-tail Fischer's Exact test was not performed for this combination since the ARI scores for both of the storage times were all 3. (Table 21- Page 73)

\section{x) Clarity Brackets Bonded with Transbond}

A two-tail Fischer's Exact test showed a significant difference between the ARI scores at times of 1 and 180-days of storage when Clarity brackets were bonded with the Transbond cement ( $p<0.001$ ). The ARI scores for the 1-day storage group ranged from 0 to 3 with $64 \%$ of the scores being 3 . The ARI score for the 180-days storage group ranged from 1 to 3 with $84 \%$ of the ARI scores being 3. (Table 21- Page 73)

\section{xi) Clarity Brackets Bonded with Panavia21}

A two-tail Fischer's Exact test showed a significant difference between the ARI scores at times of 1 and 180-days of storage when Clarity brackets were bonded with Panavia21 cement $(p<0.001)$. The ARI scores for the 1-day storage group ranged from 1 to 3 with $58 \%$ of the scores being 3. The ARI score for the 180-days storage group were all 3. (Table 21Page 73)

\section{xii) Clarity Brackets Bonded with Fuji Ortho LC (conditioned)}

A two-tail Fischer's Exact test showed a significant $(p<0.001)$ difference between the ARI scores at times of 1 and 180-days of storage when Clarity brackets were bonded with Fuji Ortho LC (conditioned) cement. The ARI scores for the 1-day storage group ranged from 0 to 3 with $67 \%$ of the scores being 3 . The ARI scores for the 180-days storage group were all 3. (Table 21- Page 73) 
Table 21: The Effects of Storage Time on ARI Scores

\begin{tabular}{|c|c|c|c|c|c|c|c|c|c|c|c|c|c|c|}
\hline $\begin{array}{l}\text { Bracket } \\
\text { Type }\end{array}$ & $\begin{array}{l}\text { Bondin } \\
\text { Protoc }\end{array}$ & & \multicolumn{4}{|c|}{$\begin{array}{l}\text { Transbond }^{\prime} \\
\text { Conditioned with } \\
\text { 37\% Phosphoric Acid, } \\
\text { dry }\end{array}$} & \multicolumn{4}{|c|}{$\begin{array}{l}\text { Panavia }^{6} \\
\text { Self-etching Acidic } \\
\text { Primer, dry }\end{array}$} & \multicolumn{4}{|c|}{$\begin{array}{l}\text { Fuji Ortho LC } \\
\text { Conditioned with } \\
10 \% \text { Polyacrylic Acid, } \\
\text { wet }\end{array}$} \\
\hline \multirow[t]{4}{*}{ Speedt } & & $\begin{array}{l}\text { ARI } \\
\%\end{array}$ & 0 & 1 & 2 & 3 & $\mathbf{0}$ & 1 & 2 & 3 & 0 & 1 & 2 & 3 \\
\hline & $\begin{array}{l}\text { Storage } \\
\text { Time }\end{array}$ & & - & $17 \%$ & $33 \%$ & $50 \%$ & $\begin{array}{r}100 \\
\%\end{array}$ & $\cdot$ & - & - & - & $25 \%$ & $50 \%$ & $25 \%$ \\
\hline & & 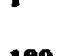 & - & $25 \%$ & $33 \%$ & $42 \%$ & $76 \%$ & $16 \%$ & $8 \%$ & - & - & $17 \%$ & $25 \%$ & $58 \%$ \\
\hline & & & \multicolumn{4}{|c|}{$\mathbf{P}=0.329$} & \multicolumn{4}{|c|}{$\mathrm{P}<0.001$} & \multicolumn{4}{|c|}{$P<0.001$} \\
\hline \multirow[t]{4}{*}{ Time $^{3}$} & & ARI & $\mathbf{0}$ & 1 & 2 & 3 & $\mathbf{0}$ & 1 & 2 & 3 & $\mathbf{0}$ & 1 & 2 & 3 \\
\hline & Storage & & - & - & $67 \%$ & $33 \%$ & $\cdot$ & $58 \%$ & $25 \%$ & $17 \%$ & - & $\cdot$ & $42 \%$ & $58 \%$ \\
\hline & (Days) & & - & - & $17 \%$ & $83 \%$ & - & $66 \%$ & $17 \%$ & $17 \%$ & - & - & $8 \%$ & $92 \%$ \\
\hline & & & \multicolumn{4}{|c|}{$P<0.001$} & \multicolumn{4}{|c|}{$P=0.385$} & \multicolumn{4}{|c|}{$P<0.001$} \\
\hline \multirow[t]{3}{*}{ Transcend ${ }^{2}$} & & ARI & 0 & 1 & 2 & 3 & $\mathbf{0}$ & 1 & 2 & 3 & $\mathbf{0}$ & 1 & 2 & 3 \\
\hline & $\begin{array}{c}\text { Storage } \\
\text { Time }\end{array}$ & 1 & - & - & $17 \%$ & $83 \%$ & - & $\cdot$ & - & $\begin{array}{l}100 \\
\%\end{array}$ & - & $\cdot$ & - & $\begin{array}{r}100 \\
\%\end{array}$ \\
\hline & (tways) & 180 & - & - & $8 \%$ & $92 \%$ & - & - & - & 100 & - & - & - & 100 \\
\hline \multirow[t]{4}{*}{ Clarity } & & ARI & $\mathbf{0}$ & 1 & 2 & 3 & $\mathbf{0}$ & 1 & 2 & 3 & $\mathbf{0}$ & 1 & 2 & 3 \\
\hline & Storage & & - & $17 \%$ & $17 \%$ & $64 \%$ & - & $17 \%$ & $25 \%$ & $58 \%$ & $8 \%$ & $\cdot$ & $25 \%$ & $67 \%$ \\
\hline & (Uays) & 180 & $8 \%$ & $8 \%$ & 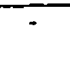 & $84 \%$ & - & - & - & $\begin{array}{r}100 \\
\%\end{array}$ & - & - & - & $\begin{array}{r}100 \\
\%\end{array}$ \\
\hline & & & \multicolumn{4}{|c|}{$P<0.001$} & \multicolumn{4}{|c|}{$P<0.001$} & \multicolumn{4}{|c|}{$P<0.001$} \\
\hline
\end{tabular}

P $<0.05$ denotes statistical significance 


\section{THE RELATIONSHIP BETWEEN SBS AND ARI SCORES DISTRIBUTION FOR VARIOUS BRACKET BASE-CEMENT COMBINATIONS (1 AND 180-DAYS OF STORAGE)}

In general, when comparing ARI scores and corresponding SBS of each bracket basecement combination, there were no consistent associations between ARI scores and SBS. $44 \%$ ( 7 out of 16) of the combinations tested after 1-day of storage showed a significant association between ARI scores and SBS values. Of these associations $57 \%$ (4 out of 7) were positive associations and $43 \%$ were negative. The groups in which a positive association was noted higher ARI scores were associated with higher mean SBS values. The groups in which a negative association was noted higher ARI scores were associated with a reduction in SBS. 17\% (2 out of 12) of the combinations tested after 180-days of storage showed a significant negative association between the ARI scores and the SBS values.

i) Speed Brackets Bonded with Transbond Cement (1 and 180-days of Storage)

The results of the linear regression test showed that there was no significant relationship between the ARI scores and SBS for Speed brackets bonded with Transbond cement tested after 1 or 180-days of storage. (Table 22- Page 75)

ii) Speed Brackets Bonded with Panaviaz1 Cement (1 and 180-days of Storage)

The results of the linear regression test showed that there was no significant relationship between the ARI scores and SBS for Speed brackets bonded with Panavia21 cement regardless of the storage time. (Table 22- Page 75)

iii) Speed Brackets Bonded with Fuji Ortho LC (conditioned) Cement (1 and 180-days of Storage)

The results of the linear regression test showed that there was a statistically significant relationship between the ARI scores and SBS for Speed brackets bonded with Fuji Ortho LC (conditioned) cement that were tested after 1 -day $(p=0.015)$. For this group higher ARI 
scores were associated with an increase in SBS. No significant association was noted for the groups tested after 180-days of storage. (Table 22- Page 75)

iv) Speed Brackets Bonded with Fuji Ortho LC (not-conditioned) Cement (1-day of Storage)

The results of the linear regression test showed that there was no significant relationship between the ARI scores and SBS for Speed brackets bonded with Fuji Ortho LC (notconditioned) cement. (Table 22- Page 75)

Table 22: Mean SBS and Corresponding ARI Scores Distribution for Speed Brackets Bonded with Different Cements (1 and 180-Days of Storage)

\begin{tabular}{|c|c|c|c|c|c|c|c|c|c|c|}
\hline \multirow{2}{*}{$\begin{array}{l}\text { Bracket Base- } \\
\text { Cement } \\
\text { Combinations }\end{array}$} & \multirow{2}{*}{$\begin{array}{l}\text { Storage } \\
\text { Time } \\
\text { (Days) }\end{array}$} & \multicolumn{4}{|c|}{$\begin{array}{l}\text { Frequency of ARI } \\
\text { Scores } \%\end{array}$} & \multirow[t]{2}{*}{$\begin{array}{l}P \\
\text { value }\end{array}$} & \multicolumn{4}{|c|}{$\begin{array}{c}\text { Mean SBS (MPa) Corresponding to } \\
\text { ARI Scores }\end{array}$} \\
\hline & & 0 & 1 & 2 & $\mathbf{3}$ & & $\mathbf{0}$ & 1 & 2 & 3 \\
\hline \multirow[t]{2}{*}{ Speed-Transbond } & 1 & - & 17 & 33 & 50 & 0.16 & - & $11.93 \pm 1.32$ & $13.59 \pm 2.79$ & $10.37 \pm 1.28$ \\
\hline & 180 & - & 25 & 33 & 42 & 0.33 & - & $14.71 \pm 2.96$ & $15.08 \pm 2.09$ & $12.63 \pm 3.84$ \\
\hline \multirow[t]{2}{*}{ Speed-Panavia21 } & 1 & 100 & - & - & - & - & $9.35=1.16$ & - & - & - \\
\hline & 180 & 76 & 16 & 8 & - & 0.84 & $8.94 \pm 1.33$ & $7.55 \pm 1.04$ & $9.69 \pm 0$ & - \\
\hline \multirow{2}{*}{$\begin{array}{l}\text { Speed-Fuji Ortho } \\
\text { (conditioned) }\end{array}$} & 1 & - & 25 & so & 25 & $0.015^{\star}$ & & $10.27 \pm 2.44$ & $12.59 \pm 0.89$ & $14.69 \pm 0$ \\
\hline & 180 & - & 17 & 25 & 58 & 0.12 & - & $13.7 \pm 0.3$ & $14.36 \pm 1.76$ & $11.69 \pm 2.08$ \\
\hline $\begin{array}{l}\text { Speed-Fuji Ortho } \\
\text { (not-conditioned) }\end{array}$ & 1 & 42 & - & 58 & - & 0.19 & $5.28=1.68$ & - & $7.69 \pm 3.9$ & - \\
\hline
\end{tabular}

$\star$ P<0.05 denotes statistical significance $P$ values denote association between ARI scores and SBS

v) Time Brackets Bonded with Transbond Cement (1 and 180-days of Storage)

The results of the linear regression test showed that there was a significant relationship between the ARI scores and SBS for Time brackets bonded with Transbond cement and 
tested after 1-day $(p=0.03)$ and 180 -days of storage $(p=0.044)$. For both storage groups higher ARI scores were associated with a reduction in SBS. (Table 23-Page 77)

\section{vi) Time Brackets Bonded with Panavia21 Cement (1 and 180-days of Storage)}

The results of the linear regression test showed that there was a significant relationship between the ARI scores and SBS for Time brackets bonded with Panavia21 cement that were tested after 1-day $(p=0.0055)$ and 180-days $(p=0.0035)$ of storage. For both of these groups their higher ARI scores were associated with a reduction in SBS. (Table 23- Page 77)

\section{vii) Time Brackets Bonded with Fuji Ortho LC (conditioned) Cement (1 and 180-days of Storage)}

The results of the linear regression test showed that there was a significant relationship between the ARI scores and SBS for Time brackets bonded with Fuji Ortho LC (conditioned) cement that were tested after 1-day of storage $(p=0.0017)$. For this group higher ARI scores were associated with a reduction in SBS. There was no significant association between the ARI scores and SBS for the group tested after 180-days of storage. (Table 23- Page 77)

\section{viii) Time Brackets Bonded with Fuji Ortho LC (not-conditioned) Cement (1-day of Storage)}

The results of the linear regression test showed that there was a significant relationship between the ARI scores and SBS for Time brackets bonded with Fuji Ortho LC (notconditioned) cement that were tested after 1-day of storage $(p=0.0015)$. For this group higher ARI scores were associated with an increase in SBS. (Table 23- Page 77) 
Table 23: Mean SBS and Corresponding ARI Scores Distribution for Time Brackets Bonded with Different Cements (1 and 180-Days of Storage)

\begin{tabular}{|c|c|c|c|c|c|c|c|c|c|c|}
\hline \multirow{2}{*}{$\begin{array}{l}\text { Bracket Base- } \\
\text { Cement } \\
\text { Combinations }\end{array}$} & \multirow{2}{*}{$\begin{array}{l}\text { Storage } \\
\text { Time } \\
\text { (Days) }\end{array}$} & \multicolumn{4}{|c|}{$\begin{array}{l}\text { Frequency of ARI } \\
\text { Scores } \%\end{array}$} & \multirow[t]{2}{*}{$\begin{array}{l}\text { P } \\
\text { value }\end{array}$} & \multicolumn{4}{|c|}{$\begin{array}{c}\text { Mean SBS (MPa) Corresponding to } \\
\text { ARI Scores }\end{array}$} \\
\hline & & 0 & 1 & 2 & 3 & & 0 & 1 & 2 & 3 \\
\hline \multirow[t]{2}{*}{ Time-Transbond } & 1 & - & - & 67 & 33 & $0.03^{*}$ & - & - & $13.87 \pm 2.02$ & $10.50 \pm 2.52$ \\
\hline & 180 & - & - & 17 & 83 & $0.044^{*}$ & - & - & $16.17 \pm 0.10$ & $11.28 \pm 2.9$ \\
\hline \multirow[t]{2}{*}{ Time-Panavia21 } & 1 & - & 58 & 25 & 17 & $0.0055^{*}$ & - & $11.64 \pm 0.91$ & $11.12 \pm 0.8$ & $9.1 \pm 0.43$ \\
\hline & 180 & - & 66 & 17 & 17 & $0.0035^{*}$ & - & $10.4 \pm 0.81$ & $7.85 \pm 0.42$ & $8.2 \pm 0.5$ \\
\hline \multirow{2}{*}{$\begin{array}{l}\text { Time-Fuji Ortho } \\
\text { (conditioned) }\end{array}$} & 1 & - & - & 42 & 58 & $0.0017 *$ & - & - & $10.04 \pm 0.69$ & $7.41 \pm 1.25$ \\
\hline & 180 & - & - & 8 & 92 & 0.17 & $\cdot$ & - & $10.7=0$ & $8.23 \pm 1.6$ \\
\hline $\begin{array}{l}\text { Time-Fuji Ortho } \\
\text { (not-conditioned) }\end{array}$ & 1 & 42 & 25 & 33 & - & $0.0015 *$ & $2.51=1.54$ & $6.11 \pm 1.42$ & $6.66 \pm 0.93$ & - \\
\hline
\end{tabular}

*P<0.05 denotes statistical significance

$P$ values denote association between ARI scores and SBS

ix) Transcend Brackets bonded with Transbond Cement (1 and 180-days of Storage)

The results of the linear regression test showed that there was no significant relationship between the ARI scores and SBS for Transcend brackets bonded with Transbond cement that were tested after 1 and 180-days of storage. (Table 24- Page 78)

x) Transcend Brackets Bonded with Panavia21 Cement (1 and 180-days of Storage)

The results of the linear regression test showed that there was no significant relationship between the ARI scores and SBS for Transcend brackets bonded with Panavia2 1 cement that were tested after 1 and 180-days of storage. For both of these groups all of the ARI scores were 3 and independent of SBS. (Table 24- Page 78) 
x) Transcend Brackets Bonded with Fuji Ortho LC (conditioned) Cement (1 and 180-days of Storage)

The results of the linear regression test showed that there was no significant relationship between the ARI scores and SBS for Transcend brackets bonded with Fuji Ortho LC (conditioned) cement that were tested after 1 and 180-days of storage. For both of these groups all of the ARI scores were 3 and they were independent of SBS. (Table 24)

\section{xii) Transcend Brackets Bonded with Fuji Ortho LC (not-conditioned) Cement (1-day of Storage)}

The results of the linear regression test showed that there was no significant relationship between the ARI scores and SBS for Transcend brackets bonded with Fuji Ortho LC (notconditioned) cement that were tested after 1-day of storage. (Table 24)

\section{Table 24: Mean SBS and Corresponding ARI Scores Distribution for Transcend Brackets Bonded with Different Cements (1 and 180-Days of Storage)}

\begin{tabular}{|c|c|c|c|c|c|c|c|c|c|c|}
\hline \multirow{2}{*}{$\begin{array}{l}\text { Bracket Base- } \\
\text { Cement } \\
\text { Combination }\end{array}$} & \multirow{2}{*}{$\begin{array}{l}\text { Storage } \\
\text { Time } \\
\text { (Days) }\end{array}$} & \multicolumn{4}{|c|}{$\begin{array}{l}\text { Frequency of ARI } \\
\text { Scores } \%\end{array}$} & \multirow[t]{2}{*}{$P$ value } & \multicolumn{4}{|c|}{$\begin{array}{c}\text { Mean SBS (MPa) Corresponding to } \\
\text { ARI Scores }\end{array}$} \\
\hline & & 0 & 1 & 2 & 3 & & $\mathbf{0}$ & 1 & 2 & 3 \\
\hline \multirow{2}{*}{$\begin{array}{l}\text { Transcend- } \\
\text { Transbond }\end{array}$} & 1 & - & - & 17 & 83 & 0.085 & - & - & $9.96=1.57$ & $16.41=4.56$ \\
\hline & 180 & - & - & 8 & 92 & 0.26 & - & - & $9.6=0$ & $15.23 \pm 4.5$ \\
\hline \multirow{2}{*}{$\begin{array}{l}\text { Transcend- } \\
\text { Panavia21 }\end{array}$} & 1 & . & - & - & 100 & - & - & - & - & $10.63 \pm 1.57$ \\
\hline & 180 & - & - & - & 100 & - & - & - & - & $8.32 \pm 1.5$ \\
\hline \multirow{2}{*}{$\begin{array}{l}\text { Transcend-Fuji } \\
\text { Ortho LC } \\
\text { (conditioned) }\end{array}$} & 1 & - & - & - & 100 & - & - & - & - & $10.30 \pm 2.18$ \\
\hline & 180 & - & . & . & 100 &. & - & - & - & $9.84 \pm 2.07$ \\
\hline $\begin{array}{l}\text { Transcend-Fuji } \\
\text { Ortho LC } \\
\text { (not-conditioned) }\end{array}$ & I & 76 & 8 & 8 & 8 & 0.46 & $7.99=1.89$ & $7.62 \pm 0$ & $7.90 \pm 0$ & $5.80 \pm 0$ \\
\hline
\end{tabular}


xii) Clarity Brackets Bonded with Transbond Cement (1 and 180-days of Storage)

The results of the linear regression test showed that there was a significant relationship between the ARI scores and SBS for Clarity brackets bonded with Transbond cement that were tested after 1 -day of storage $(p=0.009)$. For this group higher ARI scores were associated with an increase in SBS. No significant association was noted for the group tested after 180-days of storage. (Table 25- Page 80)

\section{xiii) Clarity Brackets Bonded with Panavia21 Cement (1 and 180-days of Storage)}

The results of the linear regression test showed that there was no significant relationship between the ARI scores and SBS for Clarity brackets bonded with Panavia21 cement that were tested after 1 and 180-days of storage. (Table 25-Page 80)

\section{xiv) Clarity Brackets Bonded with Fuji Ortho LC (conditioned) Cement (1 and 180-days of Storage)}

The results of the linear regression test showed that there was a statistically significant relationship between the ARI scores and SBS for Clarity brackets bonded with Fuji Ortho LC (conditioned) cement that were tested after 1-day of storage $(p=0.02)$. For this group higher ARI scores were associated with an increase in SBS. No significant association was noted for the group tested after 180-days of storage. For this group all the scores were 3 and they were independent of SBS. (Table 25-Page 80)

\section{xv) Clarity Brackets Bonded with Fuji Ortho LC (not-conditioned) Cement (1- day of Storage)}

The results of the linear regression test showed that there was no significant relationship between the ARI scores and the mean SBS for Clarity brackets bonded with Fuji Ortho LC (not-conditioned) cement that were tested after 1-day of storage. (Table 25-Page 80) 
Table 25: Mean SBS and Corresponding ARI Scores Distribution for Clarity Brackets Bonded with Different Cements (1 and 180-Days of Storage)

\begin{tabular}{|c|c|c|c|c|c|c|c|c|c|c|}
\hline \multirow{2}{*}{$\begin{array}{l}\text { Bracket Base- } \\
\text { Cement } \\
\text { Combination }\end{array}$} & \multirow{2}{*}{$\begin{array}{l}\text { Storage } \\
\text { Time } \\
\text { (Days) }\end{array}$} & \multicolumn{4}{|c|}{$\begin{array}{l}\text { Frequency of ARI } \\
\text { Scores } \%\end{array}$} & \multirow[t]{2}{*}{$\begin{array}{l}\text { P } \\
\text { value }\end{array}$} & \multicolumn{4}{|c|}{$\begin{array}{l}\text { Mean SBS (MPa) Corresponding to } \\
\text { ARI Scores }\end{array}$} \\
\hline & & $\mathbf{0}$ & 1 & 2 & 3 & & 0 & 1 & 2 & 3 \\
\hline \multirow{2}{*}{$\begin{array}{l}\text { Clarity- } \\
\text { Transbond }\end{array}$} & $\mathbf{I}$ & - & 17 & 17 & 64 & $0.009 *$ & - & $6.93 \pm 0.93$ & $12.85 \pm 4.5$ & $15.12 \pm 3.19$ \\
\hline & 180 & 8 & 8 & - & 84 & 0.49 & $10.62=0$ & $9.66=0$ & - & $12.20 \pm 3.59$ \\
\hline \multirow{2}{*}{$\begin{array}{l}\text { Clarity - } \\
\text { Panavia21 }\end{array}$} & 1 & - & 17 & 25 & 58 & 0.36 & - & $6.70 \pm 0.98$ & $10.80=1.92$ & $9.69 \pm 3.5$ \\
\hline & 180 & - & - & - & 100 & - & - & - & - & $8.64 \pm 2.46$ \\
\hline \multirow{2}{*}{$\begin{array}{l}\text { Clarity -Fuji } \\
\text { Ortho LC } \\
\text { (conditioned) }\end{array}$} & I & 8 & - & 25 & 67 & $0.02^{*}$ & $5.41 \pm 0$ & - & $8.17 \pm 1.57$ & $11.13 \pm 2.56$ \\
\hline & 180 & - & - & - & 100 & - & - & - & - & $10.13 \pm 2.58$ \\
\hline $\begin{array}{l}\text { Clarity- Fuji } \\
\text { Ortho LC } \\
\text { (not-conditione }\end{array}$ & 1 & 92 & - & - & 8 & 0.46 & $5.22+1.95$ & - & - & $3.66 \pm 0$ \\
\hline
\end{tabular}

$\star \mathbf{P}<0.05$ denotes statistical significance

$P$ values denote association between $A R I$ scores and SBS 
11. THE EFFECT OF CONDITIONING THE ENAMEL WITH $10 \%$ POL YACRYLIC ACID ON THE MEAN SBS \& ARI SCORES OF BRACKETS BONDED WITH FUJI ORTHO LC CEMENT

i) The Effects of $10 \%$ Polyacrylic Acid Conditioning on Mean SBS

Table 26: Comparison of Mean SBS of Brackets Bonded with Fuji Ortho LC with and without Enamel Conditioning

\begin{tabular}{|l|l|l|l|}
\hline $\begin{array}{l}\text { Bracket Base } \\
\text { Type }\end{array}$ & $\begin{array}{l}\text { Mean SBS O SD } \\
\text { Enamel } \\
\text { conditioned }\end{array}$ & $\begin{array}{l}\text { Mean SBS } \pm \text { SD } \\
\text { Enamel not } \\
\text { conditioned }\end{array}$ & P Value \\
\hline Speed & $11.79 \pm 2.2$ & $6.47 \pm 3.13$ & $0.0001^{\star}$ \\
Time & $8.50 \pm 1.69$ & $4.80 \pm 2.36$ & $\mathbf{0 . 0 9 0 7 ^ { * }}$ \\
Transcend & $10.30 \pm 2.18$ & $7.77 \pm 1.73$ & $\mathbf{0 . 0 2 0 0 ^ { * }}$ \\
Clarity & $9.91 \pm 2.90$ & $5.09 \pm 1.9$ & $\mathbf{0 . 0 0 0 1 ^ { * }}$ \\
\hline
\end{tabular}

$\star \mathbf{P}<0.05$ denotes statistical significance

The omission of the polyacrylic acid conditioning step when bonding brackets with Fuji Ortho LC cement resulted in a significant reduction in mean SBS for all of the bracket base types tested in this study. However, this reduction in bond strength was clinically unacceptable only for Time brackets bonded with Fuji Ortho LC. The mean SBS for the Time brackets bonded with Fuji Ortho LC and without conditioning the enamel was 4.8 $\pm 2.36 \mathrm{MPa}$ and this falls below the acceptable range $(8 \pm 3 \mathrm{MPa})$. As a result this protocol was not included in the long-term storage part of this study. 
ii) The Effects of Polyacrylic Acid Conditioning on ARI Scores Distribution

Table 27: Comparison of ARI Scores Distribution and the Mean ARI Scores of Brackets Bonded with Fuji Ortho LC Cement

\begin{tabular}{|c|c|c|c|c|c|c|c|c|c|c|c|}
\hline \multirow[t]{2}{*}{$\begin{array}{l}\text { Bracket } \\
\text { Type }\end{array}$} & \multicolumn{5}{|c|}{$\begin{array}{r}\text { Enamel conditioned } \\
\text { ARI Distribution }\end{array}$} & \multicolumn{5}{|c|}{$\begin{array}{l}\text { Enamel not conditioned } \\
\text { ARI Distribution }\end{array}$} & \multirow[t]{2}{*}{$\begin{array}{l}\text { P } \\
\text { Value }\end{array}$} \\
\hline & $\begin{array}{l}\text { Mean } \\
\text { ARI }\end{array}$ & 0 & 1 & 2 & 3 & $\begin{array}{l}\text { Mean } \\
\text { ARI }\end{array}$ & 0 & 1 & 2 & 3 & \\
\hline Speed & $2.0 \pm 0.65$ & $\mathbf{0}$ & 25 & 50 & 25 & $1 \pm 1.04$ & 42 & 0 & 58 & 0 & $0.001 *$ \\
\hline Time & $2.58 \pm 0.51$ & 0 & 0 & 42 & 58 & $0.92 \pm 0.90$ & 42 & 25 & 33 & 0 & $0.001 *$ \\
\hline Transcend & $3 \pm 0$ & 0 & 0 & 0 & 100 & $0.5 \pm 1.0$ & 76 & 8 & 8 & 8 & $0.001 *$ \\
\hline Clarity & $2.5 \pm 0.90$ & 8 & 0 & 25 & 67 & $0.25 \pm 0.87$ & 92 & 0 & 0 & 8 & $0.001 *$ \\
\hline
\end{tabular}

* P<0.05 denotes statistical significance

Polyacrylic acid conditioning of the enamel had a significant effect on the distribution of ARI scores after the debonding of all the bracket base types that were bonded with Fuji Ortho LC cement. For all the bracket base types tested, there was a significant shift from ARI scores of 2 and 3 to ARI scores of 0 and 1 when the enamel was not conditioned. This indicates that, acid conditioning caused the bond failures to occur mostly at the bracket basecement interface (favorable) compared to not conditioning. The latter resulted in bond failures at the enamel-cement interface (unfavorable).

In summary, acid conditioning had a significant and favorable effect on the type of bond failures of all the bracket base types bonded with Fuji Ortho LC cement with the result that the effect of not conditioning enamel was eliminated from the long-term storage part of the study. 


\section{COMPATIBILITY BETWEEN BRACKET BASE DESIGN AND THE CEMENT PARTICLE SIZE}

Scanning electron microscopy (SEM) micrographs of each specimen were obtained at magnifications of $\times 25, \times 100$ and $\times 1000$. The SEM micrographs are shown in Figure 7. (Page 84). The $x 1000$ magnification SEM micrographs were used to measure the average particle size of each cement. The micrographs of each bracket base surface were matched against the three cements at the same magnification. Visual analysis was made to investigate the compatibility of each bracket base surface characteristic with the cement particle size and the following observations were made: (Table 28)

Table 28. Compatibility of Different Bracket Base Designs and the Particle Size of Various Cements

\begin{tabular}{|l|l|l|l|}
\hline Cement Type & Particle Size & \multicolumn{1}{|c|}{ Description } & \multicolumn{1}{|c|}{$\begin{array}{l}\text { Compatibility } \\
\text { with Bracket- } \\
\text { Bases }\end{array}$} \\
\hline Panavia21 & $1-9$ microns & $\begin{array}{l}\text { Particles smaller than that of Fuji Ortho LC } \\
\text { and Transbond and more uniformly distributed }\end{array}$ & $\begin{array}{l}\text { Compatible with } \\
\text { all bracket base } \\
\text { types }\end{array}$ \\
\cline { 2 - 4 } Fuji Ortho LC & $1.5-27$ microns & $\begin{array}{l}\text { Quite similar in particle size and distribution } \\
\text { to Transbond }\end{array}$ & $\begin{array}{l}\text { Compatible with } \\
\text { all bracket base } \\
\text { types }\end{array}$ \\
\cline { 2 - 5 } & $1.5-27$ microns & $\begin{array}{l}\text { Quite similar in particle size and distribution } \\
\text { to Fuji Ortho LC }\end{array}$ & $\begin{array}{l}\text { Compatible with } \\
\text { all bracket base } \\
\text { types }\end{array}$ \\
\cline { 2 - 5 } & &
\end{tabular}



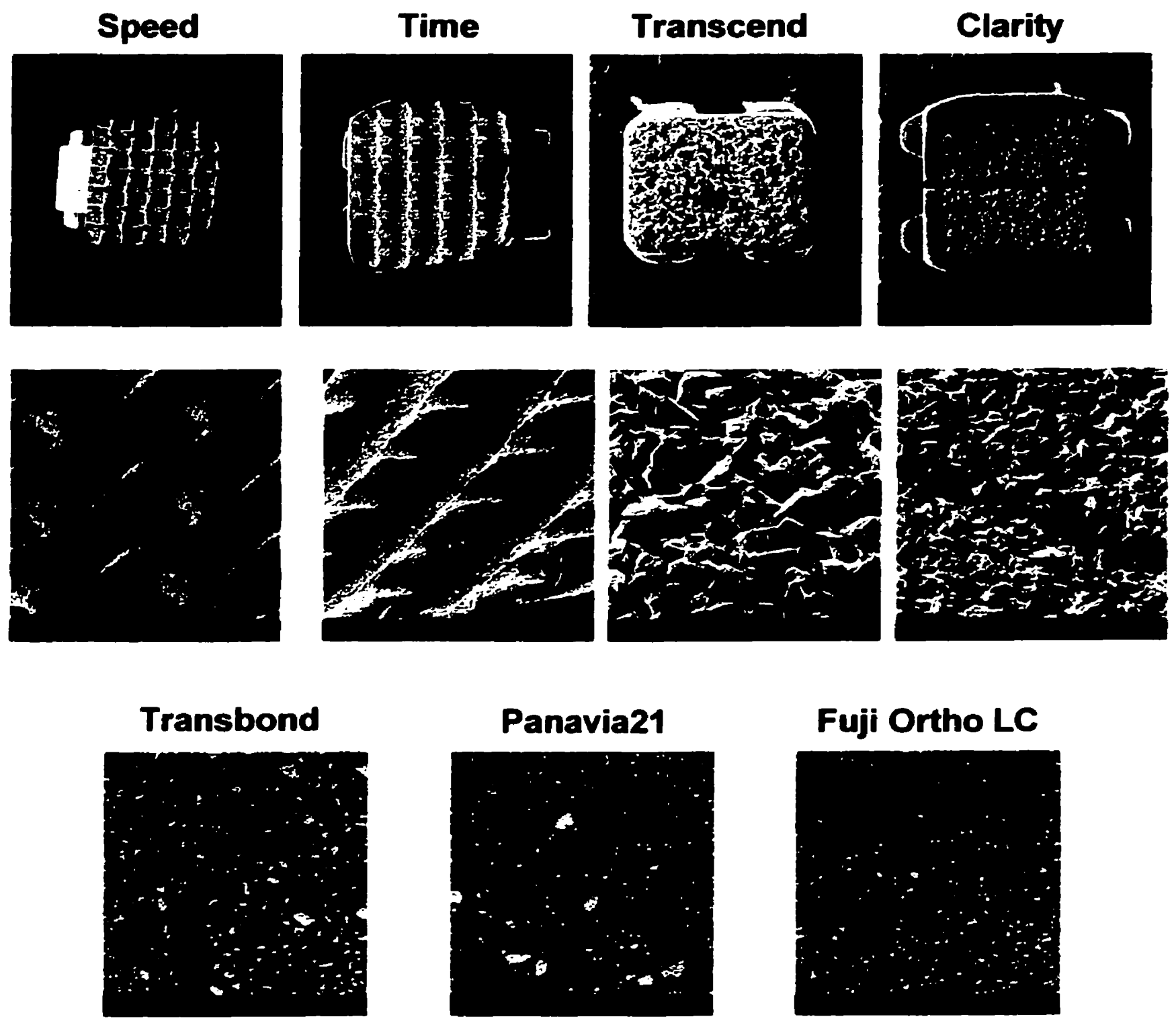

Figure 7: Compatibility of Different Bracket Base Designs and Particle Size of Various Cements

Top Row: SEM view of bracket bases at $20 \mathrm{X}$ magnification Middle Row: SEM view of bracket bases at 100X magnification Bottom Row: SEM view of different cements at $100 \mathrm{X}$ magnification 


\section{ERROR STUDY}

\section{i) Tests of Reliability for ARI Scores Assigned by Two Different Investigators}

To assess the consistency in assigning ARI scores between two different investigators an unweighted Kappa (K) statistics was carried out. This is a useful measure for quantifying agreement beyond chance between assessments of the same variable. $K$ value of zero represents random agreement, and 1.00 represents perfect agreement. The following ranges have been suggested for interpretation of Kappa: (Fleiss 1979)

0.40 and below represents poor agreement beyond chance, 0.40-0.75 represents fair to good agreement, and 0.75 and above represents excellent agreement. The Kappa statistics was calculated to be equal to $\mathbf{0 . 8 3}$, which denotes excellent agreement between the two operators in assigning ARI scores.

Table 29: ARI Scores Assigned by Two Different Investigators

\begin{tabular}{|c|c|c|c|c|c|}
\hline \multirow{5}{*}{ 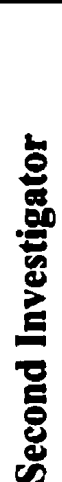 } & \multicolumn{5}{|c|}{ Principal Investigator } \\
\hline & ARI & $\mathbf{0}$ & 1 & 2 & 3 \\
\hline & $\mathbf{0}$ & 2 & $\mathbf{0}$ & $\mathbf{0}$ & $\mathbf{0}$ \\
\hline & 1 & $\mathbf{0}$ & $\mathbf{0}$ & $\mathbf{0}$ & 0 \\
\hline & 2 & $\mathbf{0}$ & $\mathbf{0}$ & 2 & 1 \\
\hline & 3 & $\mathbf{0}$ & 0 & $\mathbf{0}$ & 5 \\
\hline
\end{tabular}

Un-weighted Kappa $=0.83$ 
ii) Test of Reliability for ARI Scores Assigned by One Investigator at Two Different Times

To assess the variability between successive assignments of ARI scores by the same investigator, an un-weighted Kappa statistics was carried out. In this investigation the $\mathrm{K}$ was found to be equal to one, which accounts for excellent consistency in assigning ARI scores by the principal investigator at two different times.

Table 30: ARI Scores Assigned by the Principal Investigators at Two Different Times

\begin{tabular}{|c|c|c|c|c|c|}
\hline \multirow{6}{*}{ 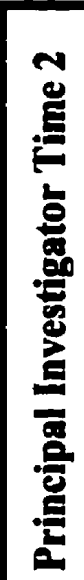 } & \multicolumn{5}{|c|}{ Principal Investigator Time 1} \\
\hline & ARI & $\mathbf{0}$ & 1 & 2 & 3 \\
\hline & $\mathbf{0}$ & 2 & $\mathbf{0}$ & 0 & $\mathbf{0}$ \\
\hline & 1 & $\mathbf{0}$ & $\mathbf{0}$ & 0 & 0 \\
\hline & 2 & $\mathbf{0}$ & 0 & 2 & 0 \\
\hline & 3 & ( & $\mathbf{0}$ & 0 & 6 \\
\hline
\end{tabular}

Un-weighted Kappa=1.0 


\section{DISCUSSION}

\section{Clinical Acceptability of Various Bracket Base-Cement Combinations}

Bond failure and the possibility of damage to enamel subsequent to acid conditioning and debonding of the brackets both during and after orthodontic treatment remains a major concern despite the advancements in the field of bonding. Bracket bond failure is an orthodontic emergency that is inconvenient for both the patient and the clinician since it is costly and may prolong the course of treatment. An acceptable orthodontic bonding system should be strong enough to withstand orthodontic and chewing forces and be easily removed with minimal or no damage to enamel. Reynolds (1975) postulated that clinical success could be achieved with cements that provide in vitro minimum bond strengths of approximately 5.0 MPa. To maintain enamel integrity, the ideal failure site in debonding should be between the bracket base and the cement (Sinha et al., 1995), although failure at bracket-base cement interface increases the difficulty of enamel clean up (Brown, 1978; Pus, 1980). Clinicians must take great care when removing remaining cement following debonding to avoid gouging, scratching, or pitting of the enamel surface.

In this investigation bracket base-cement combinations that produced mean SBS in the range of 5-11 MPa with the majority of ARI scores being 2 and/or 3 were deemed clinically acceptable. If the bond strength for a combination was greater than $11 \mathrm{MPa}$, provided that the bond failure pattern was acceptable, a SBS of more than $11 \mathrm{MPa}$ for a bracket basecement combination was considered clinically acceptable. In this investigation in order to determine an ideal combination the ease of the bonding procedure (clinical practicality), performance over time, iatrogenic damage to enamel due to the bonding procedure (acid conditioning) and the protective traits of the bonding cements (fluoride release) were also taken into account.

In this study, 16 and 12 different bracket base-cement combinations were tested after 1 and 180-days of storage respectively. After 1-day of storage $69 \%$ (11 out of 16) of the bracket base-cement combinations were clinically acceptable. The bracket base-cement combinations that performed poorly were Speed-Panavia21, Time-Panavia21, Time-Fuji 
Ortho LC (not-conditioned), Transcend-Fuji Ortho LC (not-conditioned), and Clarity-Fuji Ortho LC (not-conditioned) combinations. (Table 6- Page 50) Only the Time-Fuji Ortho LC (not-conditioned) combination produced clinically unacceptable SBS (4.8 $\pm 2.36 \mathrm{MPa})$. All of the above five combinations resulted in unacceptable bond failure patterns i.e. most of the cement was left on the bracket base. The results obtained with Fuji Ortho LC (notconditioned) were in disagreement with the manufacturer's claim and the observations of Silverman et al. (1995) and Fricker (1994) that Fuji Ortho LC performs similarly with or without the use of an enamel conditioner. The results of the present study support the current opinion that advocates the use of an enamel conditioner either $10 \%$ polyacrylic acid (Beress et al., 1998; Bishara et al., 1998b; Lippitz et al., 1998; Flores et al., 1999; Millett et al., 1999; Meehan et al., 1999) or 37\% phosphoric acid (Flores et al., 1999; Bishara et al., 2000) prior to bonding with resin reinforced glass ionomer cements. Based on the poor results obtained for the majority of the bracket bases (Time, Transcend and Clarity) when bonded to not-conditioned enamel with Fuji Ortho LC cement, this bonding protocol was omitted from the long-term storage study.

Twelve different combinations were tested after 180-days of storage. The results showed that long-term storage had no clinically significant effect on the acceptability of both the bond strength and the bond failure pattern of the different bracket base-cement combinations (Tables 16, 21-Pages 63, 73). In both 1-day and 180-days storage groups only SpeedPanavia21 and Time-Panavia21 resulted in unfavorable failure patterns while producing adequate bond strengths. The possible causes for this result will be discussed later. With respect to the effects of long-term storage, the data are limited and study-dependent. Comparisons can only be made with studies that have utilized the same type of cement, bonding protocol and bracket base design. The only study closely resembling the protocol and the materials tested in this study is that of Mahal (2000). In this study metal (Speed), ceramic (Transcend) and polycarbonate (SpiritMB) brackets were bonded to bovine enamel using Phase II composite resin and GC Fuji Ortho. Both of these cements are autopolymerizing. The samples were subjected to thermocycling and long-term storage. The thermocycling period was 24-hours and 6.5-days. The long-term storage period consisted of shear bond testing at 24-hours, 7-days, and 180-days. The results indicated that long-term 
storage had a significant effect on the SBS and this effect was in turn dependent on the bracket base type, and the type of cement used. The mean SBS in the present study were considerably lower than those of Mahal (2000) at the corresponding storage periods of 24hours and 180-days. This difference is most likely due to different cement types used. In the Mahal (2000) study both the RCLC and RRGIC were of the auto-polymerizing type. It is possible that polymerization continued after the initial set so that these cements produced higher mean SBS. In the present study, long-term storage had a statistically significant effect on the SBS of 3 out of 12 bracket base-cement combinations. The SBS for the SpeedTransbond combination increased from 11.69 $\pm 2.3 \mathrm{MPa}$ to $14.05 \pm 2.84 \mathrm{MPa}$. The SBS for the Time-Panavia2 1 combination and the Transcend-Panavia21 combination decreased from $11.07 \pm 1.26 \mathrm{MPa}$ and $10.63 \pm 1.57$ to $9.61 \pm 3.5 \mathrm{MPa}$ and $8.32 \pm 1.5 \mathrm{MPa}$ respectively. However, these changes in SBS did not detract from clinical acceptability of these combinations. In the Mahal study, long-term storage (180-days) did not have a clinically significant effect on the fracture pattern of bonded brackets. The results of the present study are in agreement with this observation.

\section{The Effects of Bracket Base Design, Cement Type, Enamel Conditioning and Long-term Storage on the Mean SBS}

In general, the results of this study showed that bracket base design, cement materials, enamel conditioning and bracket base-cement combinations had a significant effect on the mean SBS.

i) The Effects of Bracket-Base Design on the Mean SBS, 1-Day vs 180-Days of Storage

In this study the Duncan's grouping showed that Transcend brackets performed significantly differently than the Time, Speed and Clarity brackets by producing the highest overall SBS. (Table 9- Page 53) When comparing the bracket base design of the Transcend and Clarity brackets (Figure 7- Page 84 ), it is evident that these brackets have different base sizes and designs. The Clarity bracket is a larger bracket, but this should not have an affect on SBS. 
Both of these brackets are of the polycrystalline aluminum oxide type with randomly oriented crystals fused on to their bases. As seen on the SEM micrographs (Figure7- Page 84), the Clarity bracket base has both a midline grove running in gingival-incisal direction, and the sizes of the crystals and recesses in between the crystals are smaller than those of Transcend bracket base. The manufacturers have incorporated the midline grove in the base of Clarity bracket to allow for flexure during debonding thus making it easier to debond. The Transcend bracket base lacks this feature. It is thought that the presence of this groove resulted in reduction in the bond strength of Clarity brackets when compared to Transcend brackets regardless of the cement type used. The Clarity bracket base also has no crystal particles around its periphery, resulting in a reduced surface area for bonding. Subsequently, lower SBS values were recorded for Clarity brackets as compared to Transcend brackets.

The metal Speed and Time bracket bases produced respective cumulative mean SBS of 9.83 MPa and 9.27 MPa, which were independent of the cement type, used. (Table 9- Page 53) These mean SBS were not statistically different from each other except that Speed bracket bases produced slightly higher mean SBS. This difference in bond strengths is due to differences in the base surface design of these brackets. The Speed bracket has a 60 -gauge, microetched foil-mesh base as compared to the Time bracket, which has a machined, integral, microetched base with mechanical undercuts (Figure 7- Page 84). As seen in the SEM micrographs (Figure 7- Page 84), the channels between the mesh of Speed bracket base are more retentive than the undercuts on the Time bracket base because they are deeper and more numerous. Sharma (1999) compared the SBS of 6 different metal bracket base designs bonded to bovine enamel using Transbond cement and tested after 1 and 24-hours. The highest 24-hour SBS she reported were achieved with Time bracket bases followed by Speed bracket bases. These findings are in agreement with the results of this study since the Time-Transbond combination produced higher SBS values than the Speed-Transbond combination. However, when comparing SBS of different bracket bases for all the different cements combined, Speed brackets performed slightly but not statistically significantly better than Time brackets for the reasons mentioned above. These latter findings are also in agreement with two other studies (Deidrich and Dickmeiss, 1984; Regan and Van Noort, 1989) which demonstrated that machined integral bases show lower bond strengths 
compared to foil-mesh bases. The over all conclusion is that bracket base design does have an effect on SBS as shown in previous studies (Reynolds and von Fraunhofer 1977; Faust et al., 1978; Thanos et al., 1979; Dickinson and Powers, 1980; Lopez, 1980; Maijer and Smith, 1981, Deidrich and Dickmeiss, 1983; Regan and Van Noort, 1989; Sharma, 1999)

The same pattern of performance was noted for both metal and ceramic bracket bases tested after 180-days of storage when compared to those tested after I-day of storage. At both storage times, Speed and Transcend bracket bases produced higher SBS than the Time and Clarity bracket bases. (Table12- Page 55)

\section{ii) The Effects of Cement Type and Enamel Conditioning on the Mean SBS}

Another variable affecting bond strength is the cement type. The SBS values for the three cements and four different bonding protocols after 1-day of storage indicated that Transbond cement performed significantly differently than Panavia21 and Fuji Ortho LC (conditioned and not- conditioned) cement (Table 10- Page 54). The cumulative mean SBS for Transbond cement was 13.29 MPa. The values for Panavia21 (10.13 MPa) and Fuji Ortho LC (conditioned, 10.13 MPa) were identical. The mean SBS for Fuji Ortho LC cement dropped significantly to $6.03 \mathrm{MPa}$ when conditioning of enamel with $10 \%$ polyacrylic acid was excluded. The bonding protocols for these cements were different in that different types of conditioners were used for each cement type. The differences in performance of these cements are not only due to their differing chemical and physical properties but also due to the differences in the modes of enamel treatment. Conditioning with $37 \%$ phosphoric acid produces a different enamel etch pattern compared with conditioning with $10 \%$ polyacrylic acid (Coups Smith, 1997; Bishara, 2000). 10\% polyacrylic acid is a milder acid than the $37 \%$ phosphoric acid. Phosphoric acid produces deeper micro-porosities than polyacrylic acid, which results in stronger retention of Transbond cement by tooth than the Fuji Ortho LC cement. As a result, the observation of higher mean SBS for Transbond cement in combination with $37 \%$ phosphoric acid is in part due to differences in adherence of Transbond and Fuji Ortho LC (conditioned) cements to enamel. The differences in bond strengths of these two cements are also in part attributed to their different chemical composition. In general, RRGIC are weaker cements than RCLC since the resin content of 
RRGIC is only $11.25 \%$ as compared to Transbond which is a BIS-GMA resin based cement. The consensus in the current literature is that RRGIC continues to produce lower but reliable bond strengths when compared to RCLC (Bishara et al., 1998b; Bishara et al., 1999b; Meehan et al., 1999; Millett, 1999). The results of this study are in agreement with the current literature. Even though bond strengths of Fuji Ortho LC with conditioner were statistically lower than Transbond, they were, however, clinically acceptable.

Another property of Fuji Ortho LC cement is that being glass ionomer cement it bonds chemically to tooth structure and the metal. The results of this study showed that a chemical adhesion to the tooth surface is not enough and that mechanical interlocking of the cement to enamel by polyacrylic acid conditioning is required for consistent clinically acceptable bond strengths. This is in agreement with the findings of Beress et al., 1998; Bishara et al., 1998b; Flores et al., 1999; Lippitz et al., 1998; Meehan et al., 1999; Millett et al., 1999. These results, however, are contrary to manufacturer's claim that conditioning with polyacrylic acid is optional and not required when bonding brackets with Fuji Ortho LC cement. In this study, the combination of Time-Fuji Ortho LC (not-conditioned) produced an unacceptable low mean bond strength, 4.7 $\pm 2.36 \mathrm{MPa}$. (Table 26- Page 81) The bond strengths of the Speed, Transcend and Clarity brackets when bonded with Fuji Ortho LC (not-conditioned) were all clinically acceptable but were significantly lower than the same brackets bonded with either Panavia21, Transbond or Fuji Ortho LC (conditioned) cements.

Panavia21 cement produced cumulative bond strengths that were less than Transbond but exactly equal to Fuji Ortho LC (conditioned). (Table 10- Page 54) Panavia21 is a low viscosity restorative RCLC with a filler content of over $70 \%$ by weight. This cement is autopolymerized in the absence of oxygen. Panavia2 1 contains the adhesive monomer MDP (10Methacryloyloxydecyl dihydrogen phosphate) that provides chemical adhesion between tooth structure and metal, silanated porcelain, or composite. (Rux et al., 1991) The reason that Panavia21 and Fuji Ortho LC (conditioned) cements provided the same cumulative mean SBS is probably due to their common unique property of chemical adherence to metal. In this study, Panavia21 did not chemically adhere to ceramic bracket bases since the bracket bases used in this study (Transcend and Clarity) were not silanated. The bond 
strength values for Panavia21 used as orthodontic cements to bond brackets to enamel is limited to one study by Bishara et al. (1998a). Bishara and colleagues (1998a) were interested in Panavia2l as an orthodontic cement because of the unique properties of its selfetching acidic primer. Unlike other conventional resin cements that require a conditioner plus a low viscosity adhesive primer prior to bonding brackets to enamel, Panavia21 system has the conditioning and priming steps combined into one step of a self-etching acidic primer. The unique characteristic of this self-etching acidic primer is that it allows the clinician to combine conditioning and priming into a single treatment step. This results in an improvement in both time and cost effectiveness to the clinician and indirectly to the patient (Bishara et al., 1998a). Bishara et al. (1998a) used Panavia21 with a self-etching acidic primer to bond metal brackets (Victory series, 3M, Unitek, Monrovia, Calif) to human molars. Their results showed that the use of acidic primers to bond brackets to the enamel provided clinically acceptable SBS $(10.4 \pm 4.4 \mathrm{MPa})$ when used with a highly (77\% by weight) filled adhesive (Panavia21). The results of the present study are in agreement with these findings in that the use of the self-etching acidic primer with Panavia21 to bond four different bracket bases produced acceptable bond strengths after 1 and 180-days of storage.

\section{iii) The Effects of Long-Term Storage on the Mean SBS of Different Cements, and Various Bracket Base-Cement Combinations}

Fuji Ortho LC cement with enamel conditioner produced a cumulative mean SBS (8.83 $\mathrm{MPa}$ ) that was significantly lower than the SBS of Transbond (13.19 MPa) and Panavia21 (10.27 MPa), after 180-days of storage. (Table 13- Page 56) In addition, the cumulative mean SBS for Transbond and Panavia21 did not change significantly after 180-days of storage. These results indicate that Transbond and Panavia21 cements, which are RCLC, perform better than Fuji Ortho LC, which is a RRGIC. The resin content of both Transbond and Panavia21 cements surpass that of Fuji Ortho LC. The improved bond strength of RRGIC as compared to conventional GIC is due to the addition of the resin. Based on the results of the present investigation it appears that the higher the resin content the stronger the cement and the better the performance over time. From the results of this study, it can be interpreted that the resin content imparts resistance to degradation and solubility over time 
in water. The reduction in bond strength following long-term storage of RRGIC may be attributed to cement degradation. Since RRGIC has less resin content compared to RCLC, it is therefore more soluble and as a consequence produces lower SBS after long-term storage in water. It is important to note, however, that these changes are not clinically significant and the rate of reduction in bond strength is very slow, and the performance of the cement over the average length of time required for orthodontic treatment is not affected.

Long-term storage had a statistically significant effect on the bond strength of 3 out of 12 bracket base-cement combinations. (Table 16- Page 63) The storage periods of 180-days resulted in statistically significant reductions in SBS of Time and Transcend brackets bonded with Panavia21 cement. There are no long-term storage data on Panavia21. It is manufacturer's recommendation that any exposed Panavia2 1 cement should be covered by Oxyguard ${ }^{\mathrm{TM}}$ since the setting reaction is anaerobic. The reason that Panavia21 cement was included in the present investigation was due to simplified step of using self-etching acidic primer and the use of Oxyguard ${ }^{\mathrm{TM}}$ would have detracted from its simplicity. It should be noted that, Bishara et al. (1998a) did not use Oxyguard ${ }^{\mathrm{TM}}$ in their bonding protocol. Another reason for omission of this step was that the thickness of cement between the bracket and the tooth is quite thin. Using the same bracket seating force used in this study, MacColl (1995) measured this interface to be $0.07 \mathrm{~mm}$ thick. For the purposes of this study, it was postulated, therefore, that the amount of potentially un-polymerised cement at the periphery of the bracket base would not affect the bond strength. The results of this study indicate that Time and Transcend brackets cemented with Panavia21 showed a reduction in bond strength after 180-days of storage. The SBS of Speed and Clarity brackets bonded with Panavia21 were not significantly affected by the storage time of 180-days. This observation can be attributed to the base design of these brackets and the amount of un-polymerised cement at the periphery, which may have dissolved in water over the period of 180-days of storage. Time and Transcend bracket bases are not as retentive when compared to Speed and Clarity bracket bases respectively. Therefore, it is possible that the poor interlocking of the cement material into the bases of Time and Transcend brackets, in combination with the presence of the un-polymerised resin at the periphery, may have accelerated cement dissolution in water 
and thus resulted in lower bond strengths over time for these bracket bases. The results of the present study can not be compared to any other studies since the Bishara et al. (1998a) study used 48-hours storage period when using Panavia21 as bracket cementing agent.

Independent of bracket base design and material, Transbond cement performed similarly with respect to SBS at both time intervals. However, if the bracket base design is included in the comparisons between SBS at the two different storage times, a difference is noted. Longterm storage resulted in an increase in SBS of Speed brackets bonded with the Transbond cement. The SBS of Time, Clarity and Transcend brackets bonded with Transbond were not affected by the long-term storage. Sharma (1999) showed that the SBS of 6 different metal bracket bases (Speed and Time included) bonded with Transbond cement increased from 1hour to 24-hours storage. The results of the present study can't be compared to that of Sharma's, since the storage periods are different. The only study using the same storage period with different cements was that conducted by Mahal (2000).

In the Mahal (2000) study, long-term storage resulted in a significant increase in the SBS of Speed, Transcend and SpiritMB brackets when they were bonded with either autopolymerising RCLC or auto-polymerising RRGIC cements. This is probably because the polymerisation of auto-polymerised cements may continue for longer periods than that of photo-polymerising cements. In the present investigation, long-term storage resulted in a significant increase in the SBS of only the Speed-Transbond combination.

There were no statistically significant differences in SBS over time for the brackets that were bonded with Fuji Ortho LC cement (conditioned). (Table 16- Page 63) These results are not in agreement with those of Mahal (2000). Mahal (2000) demonstrated an increase in the bond strengths of all the bracket types bonded with GC Fuji Ortho cement (conditioned). Once again, it should be noted that GC Fuji Ortho is an auto-polymerising cement that may have had continued polymerisation overtime.

In general, long-term storage had no clinically significant effect on the SBS of the different bracket base cement combinations used in this study. All of the 12 combinations continued 
to produce shear bond strengths, which were greater than the cut off value of 5.0 MPa. (Table 16- Page 63)

\begin{abstract}
ARI Scores
i) The Effects of Cement Type and Enamel Conditioning on ARI Scores Duncan's grouping showed that Transbond (mean ARI=2.45) and Fuji Ortho LC conditioned (mean ARI=2.5) produce the optimum ARI scores followed by Panavia21 (mean ARI=1.75) and Fuji Ortho LC not-conditioned (mean ARI=0.67) after 1-day of storage. (Table18- Page 65) The same pattern of performance was observed for each cement after 180-days of storage; Transbond (mean ARI=2.63), Fuji Ortho LC (conditioned) (mean $A R I=2.83$ ) and Panavia 21 (mean $A R I=1.96$ ). (Table 20- Page 75) Long-term storage resulted in a slight increase in ARI scores for all the cement types. These observations did not take into account the bracket-base design.
\end{abstract}

These data clearly show that enamel conditioning had a significant effect on the ARI scores. For Fuji Ortho LC cement, when the enamel surface was not conditioned all the failure patterns were unacceptable and independent of the bracket-base design. Conditioning with $10 \%$ polyacrylic acid significantly improved the failure patterns of all the bracket-base types bonded with Fuji Ortho LC cement in that the majority of the cement was left on the tooth rather than the bracket base after debonding. The results of this study show that enamel conditioning had a greater effect on the bond failure pattern of all bracket base types than the cement material itself.

ii) The Effects of Various Bracket Base-Cement Combinations on ARI Scores

a. Transbond Cement and Various Bracket Base Types

After 1-day of storage, Transbond cement produced mean ARI scores with all of the bracket base types that ranged from 2.33 to 2.83 with an overall mean of 2.45. (Table 17- Page 64) These results indicate that Transbond cement was compatible with all the bracket-base types 
tested in this study in producing clinically acceptable ARI scores where most of the cement was left on the tooth thus minimising iatrogenic enamel damage during debonding.

ARI scores distributions were more favourable for the Time bracket-base than the Speed bracket-base. Even though the majority of the ARI scores for both metal brackets were 2 and 3, Speed bracket bases had $17 \%$ of the failures occurring so that less than half of the cement was remaining on the tooth i.e. ARI score of 1 . However, all of the scores for Time brackets were 2 and 3 . This result is due to differences in the retentive capability of the bases of these two metal brackets. The Time bracket base has less retentive characteristics when compared to the meshwork of Speed bracket base. As a result, less of the cement is left on the Time bracket base and more on the tooth after debonding compared to Speed bracket base, which retains most of the cement. Long-term storage had no effect on the ARI score distribution for Speed brackets bonded with Transbond cement. In the case of the Time bracket, more ARI scores of 3 were observed after long-term storage but these differences were not clinically significant. (Table 21 - Page 73)

Transcend bracket bases bonded with Transbond cement produced higher ARI scores than Clarity bracket bases bonded with the same cement. It seems that mechanical interlocking between the Transcend bracket base and Transbond cement was less than that between the Clarity bracket base and Transbond. When comparing the base design of these brackets the Clarity bracket base is more retentive, because it has smaller particles and smaller recesses in between the particles (Figure 7- Page 84). In addition, the Clarity bracket base has a midline groove. In comparison, the Transcend bracket-base has relatively larger particles and larger recesses and no midline groove. The Clarity bracket base is more retentive than the Transcend bracket base and retains more cement during debonding, which results in poorer but acceptable ARI scores. Long-term storage had no clinically significant effect on the ARI score distribution of either of these bracket bases. (Table 21 - Page 73)

\section{b. Panavia21 Cement and Various Bracket Base Types}

Panavia21 cement produced poor and unacceptable ARI scores in combination with the metal bracket bases (Speed and Time). The ARI scores for the Speed bracket bases were all 
0 and the mean ARI score for the Time bracket base was $1.58 \pm 0.79$. Unlike Transbond cement, Panavia21 has chemical adhesive properties to metal. As a result, it appears that the bond strength (micro-mechanical interlocking plus chemical adhesion) between Panavia21 and the metal bracket bases was stronger than that between Panavia21 and enamel. As a result, most of the cement was left on the metal bracket bases. The Time bracket base produced higher ARI scores than the Speed bracket base because its base is less retentive resulting in a weaker micro mechanical bond between its base and the cement. Subsequently, more cement remained on the tooth after debonding the Time brackets compared with the Speed bracket base. Storage time had no clinically significant effect on ARI score distributions of these bracket base-cement combinations and they consistently produced poor ARI scores. (Table 21 - Page 73)

The ARI scores for both of the ceramic bracket bases at storage times of 1 and 180-days were acceptable when Panavia21 was used to bond them to enamel. Once again the ARI scores were more favourable for Transcend brackets than for Clarity brackets owing to the different bracket base designs. Panavia2l cement performed better with ceramic bracket bases than with metal bracket bases because of its chemical properties. Panavia21 bonds to metal and ceramic surfaces that are treated with silane. The ceramic bracket bases used in this study were not treated with silane. As a result, there was no chemical bonding between the ceramic bracket bases and Panavia21 so that the bond between the enamel and the cement was stronger than the micro-mechanical bond between the cement and the bracket base. As a result, more cement was left on the tooth than on the bracket base. The storage time had no clinically significant effect on the ARI scores distributions of these bracket base-cement combinations. (Table 21- Page 73)

\section{c. Fuji Ortho LC (conditioned) Cement and Various Bracket Base Types}

The same trend was observed for Fuji Ortho LC (conditioned) cement as with Transbond in that all the bracket bases resulted in clinically acceptable ARI scores with this cement. Unlike Panavia21 cement, which produced clinically unacceptable bond strengths with the metal bracket bases, Fuji Ortho LC (conditioned) resulted in acceptable ARI scores with 
both the metal and ceramic bracket bases. It appears that the chemical adhesive properties of Fuji Ortho LC cement to metal are not as strong as those of Panavia21. Time and Transcend bracket bases produced higher ARI scores than the Speed and Clarity bracket bases respectively. Once again, time had no appreciable effect on the clinical performance of these bracket base-cement combinations with respect to ARI scores. (Table 21- Page 73)

\section{d. Fuji Ortho LC (not-conditioned) Cement and Various Bracket Base Types}

All the bracket base cement combinations with Fuji Ortho LC (not-conditioned) cement produced unacceptable ARI scores due to the omission of the acid conditioning step. Therefore, regardless of bracket base type or design, less cement was left on the tooth after bracket debonding since there was no mechanical interlocking between the cement and the enamel. The chemical adhesion of the cement to enamel was very low compared to the bond between the bracket bases and the cement. The bracket base design had the same effect as recorded with other cements on the ARI scores. The same trend was observed in that Time and Transcend brackets performed better than Speed and Clarity brackets respectively.

\section{The Relationship between SBS and ARI}

In this investigation a consistent association was not demonstrated between the ARI scores and shear bond strengths in either of the storage groups. 44\% (7 out of 16) of the combinations tested after 1-day of storage showed a significant association between the ARI scores and SBS values. Of these associations, $57 \%$ ( 4 out of 7) were positive associations and $43 \%$ were negative. A positive association implied that the higher the SBS, the higher the ARI score with the result that more cement remained on the enamel after debonding. In contrast, a negative association implied that the specimens with the lower bond strengths debonded with most of the cement remaining on the tooth producing a higher ARI score. $17 \%$ ( 2 out of 12 ) of the combinations tested after 180-days of storage showed a significant negative association between the ARI scores and SBS values.

A previous study, using Transbond, Phase II, Fuji Ortho LC and GC Fuji Ortho cements to bond Speed brackets to bovine enamel, showed a positive correlation between SBS and ARI 
scores for both 1 and 7-days storage times (Coups Smith, 1997). This positive correlation implies that as the mean SBS increases, so does the ARI score. MacColl (1995) investigated the effects on SBS by sandblasting bracket base surfaces, reducing the base surface area and conditioning enamel with various acid types. In this study, the general trend was that higher shear bond strengths were associated with most of the cement remaining on the bracket base i.e. lower ARI scores (MacColl, 1995). Similarly, Sharma (1999) showed a negative linear relationship between the mean SBS and mean ARI scores. A high bond strength was correlated with a low ARI score. The results of the present study are in disagreement with all of the above findings since no consistent association was noted between the ARI score and SBS. According to O'Brien et al. (1988), the amount of cement remaining on the enamel after the debonding of bonded brackets is not related to the mean SBS of the separate interfaces but rather it is related to the bracket base characteristics and the properties of the cement used. The results of this study were in agreement with this conclusion in that SBS was not a determinant of ARI score. The ARI score as shown in this study was influenced by the modes of enamel surface treatment, the bracket base design and the chemical properties of the cement materials. The results of this study clearly indicated that bonding with Fuji Ortho LC cement without conditioning the enamel surface resulted in a significant shift in ARI scores distributions. (Table 27- Page 82) When the enamel surface was not conditioned with $10 \%$ polyacrylic acid the majority of the failures occurred at the enamelcement interface (ARI scores of 0 and 1). On the other hand, enamel conditioning with $10 \%$ polyacrylic acid resulted in failures at bracket base-cement interface. Bracket base design also had an effect on the ARI scores regardless of the cement types used; Time and Transcend bracket bases performed differently from Speed and Clarity bracket bases respectively. In addition, chemical adhesion of Panavia21 cement to metal bracket bases resulted in a shift in the ARI score distribution. For Time and the Speed bracket bases bonded with Panavia2l cement, the bond failures occurred at enamel-cement interface rather than at the bracket base-cement interface as recorded for Transbond and Fuji Ortho LC (conditioned) cement. The results of the current study indicate that enamel conditioning is the variable that most significantly affects the bond failure pattern when considering all the factors that can influence bond failure pattern, i.e. bracket base design, cement type, enamel treatment and long-term storage. 


\section{Bracket Base Design and the Cement Particle Size Compatibility}

The orthodontic literature lacks studies that are aimed at matching cement particle size with bracket base design. Currently, there is no information on the compatibility of cement particle size and bracket base design. It seems logical, however, that in order for cement particles to be micro-mechanically retained by the bracket base surface, the particle sizes should be compatible with the recesses and the undercuts on the bracket bases. The results of this study (Figure 7- Page 84) clearly demonstrated that the particle size of the Transbond, Panavia21 and Fuji Ortho LC cements were all compatible with the bracket base design of the Speed, Time, Transcend, and Clarity brackets.

\section{The Ideal Bracket Base-Cement Combinations}

In this study the clinical acceptability of each bracket base-cement combination using ARI scores and SBS values was assessed. As stated previously, a clinically acceptable system not only should produce reliable bond strengths over the course of orthodontic treatment, and minimize damage to the enamel during debonding, but should also be easy and practical to use. This system should not cause iatrogenic damage to enamel during bonding, over the course of treatment and debonding.

When considering SBS and ARI scores, both Transbond and Fuji Ortho LC cements produced clinically acceptable results with all the bracket base types. The Panavia21 cement combination with Time and Speed bracket bases resulted in unfavourable ARI scores. Similarly, Time-Panavia21 and Transcend-Panavia21 combinations produced SBS that were statistically significantly reduced over the long-term storage.

From a practical standpoint, even though Panavia2l cement required fewer steps of enamel preparation compared to the Transbond and Fuji Ortho LC cements it had, however, a low viscosity which may result in bracket slippage during the initial phases of cement polymerization. Panavia2l cement's low viscosity and bracket slippage did not pose a problem in this study because the brackets were held horizontally in place under the 
weighted guide pin of an articulator. In a clinical situation, however, it is not practical to hold a bracket onto the tooth in a vertical position while the cement is polymerizing.

Of the two acceptable cements, Transbond and Fuji Ortho LC (conditioned), Fuji Ortho LC is considered to be a superior alternative for the following reasons:

1. Fuji Ortho LC uses a milder conditioner, $10 \%$ polyacrylic acid as compared to Transbond, which requires conditioning with $37 \%$ phosphoric acid. Coups Smith (1997) showed different enamel etch patterns using 10\% polyacrylic acid compared to $37 \%$ phosphoric acid. The etch pattern created by $10 \%$ polyacrylic acid was less pronounced and not of honeycomb-like appearance as found with the $37 \%$ phosphoric acid. Based on this observation, Coups Smith (1997) suggested that polyacrylic acid creates less of a risk of damage to the enamel upon debonding and less of a risk of staining of the enamel over time, since the enamel would be less penetrated by the resin tags (Urabe, 1996).

2. Fuji Ortho LC cement, unlike the Transbond cement, is not moisture sensitive so that the brackets can be bonded to enamel that is contaminated with water and/or saliva.

3. It has been reported that less enamel damage occurs during clean up after the debonding of resin reinforced glass ionomer cements (Ostman-Andersson et al., 1993). According to Norevall et al. (1995) RRGIC can be more easily removed than RCLC at the time of debonding, since any cement remaining on the enamel surface can be desiccated by air-drying. This renders it more friable and removable (White, 1986). As a result, the likelihood of enamel damage being incurred after debonding GIC cemented brackets is less than that of those cemented with RCLC.

4. Fuji Ortho LC has the ability to release (Fox, 1990; Ashcraft et al., 1997) and absorb fluoride (Hatibovic-Kofman and Koch, 1991; Creanor et al., 1994). Glass ionomer cements release considerable amounts of fluoride and they have been shown, in vitro, to prevent demineralization of enamel (Forss and Seppa, 1990). It has also been reported that a less cariogenic flora is found in plaque deposits adjacent to GICs 
(Hallgren et al., 1992). Wright et al. (1995) studied the effect of resin-reinforced glass ionomer cement (RRGLC) on the oral microflora. Their results showed that levels of Lactobacilli and S.mutans (cariogenic bacteria) around the brackets bonded with RRGIC were reduced when compared with the conventional diacrylate resin cements.

\section{Future Research}

When this study was conducted there was no self-etching acidic primer available for orthodontic bonding purposes that would be compatible with currently available orthodontic cements. The studies of Bisahar et al. (1998a, 1999a) showed that acidic primer was only compatible with Panavia21 cement and could not be used with the Transbond cement. Recently, the Unitek Company introduced Transbond Plus Self Etching Primer ${ }^{\boldsymbol{m}}$. This system is a unit-dose system, with conditioner, primer, adhesive, and micro-brush sealed into a triple-lollipop-shaped aluminium foil package. Acid conditioning, rinsing, and e than half of the cement remaining on the tooth. The Speed brackets bonded with Panavia 21 cement produced the poorest ARI aid to produce a well defined etch pattern similar to that of phosphoric acid (3M/Unitek, California) and it forms a micro retentive bond with the treated surface. It also allows the conditioner and priming monomer to penetrate at the same time, avoiding potential technique errors. Also no light polymerisation is required and moisture contamination is tolerated. This system is specifically designed for orthodontic bonding and is compatible with the Transbond cement. An orthodontic adhesive system such as this is of tremendous value since it can save valuable chair side time.

It is imperative that the manufacturer's claims are tested, as there is no independent study conducted using this adhesive system. The protocol developed in this study can be used to evaluate the clinical acceptability of this product. 


\section{CONCLUSIONS}

- SBS and ARI scores of $69 \%(11 / 16)$ and $83 \%$ (10/12) of bracket base-cement combinations were acceptable when tested after 1-day and 180-days of storage respectively.

- Bracket base design had a significant effect on the SBS at both 1 and 180-days of storage time. Speed and Transcend brackets produced higher SBS than Time and Clarity brackets respectively.

- Bracket base-cement combination had a significant effect on the ARI scores at both 1 and 180-days of storage time. Time and Transcend brackets in combination with Transbond and Fuji Otho LC (conditioned and not-conditioned) cements produced higher ARI scores than the Speed and Clarity brackets. Metal brackets (Time and Speed) in combination with Panavia21 cement resulted in lower ARI scores than the ceramic brackets (Transcend and Clarity) irrespective of bracket base design. This was due to chemical adhesion of this cement to the surface of the metal.

- Cement type and the enamel treatment (conditioning or not-conditioning) had a significant effect on the SBS and ARI scores. Transbond produced the highest mean SBS at both 1 and 180-days of storage followed by Panavia21 and Fuji Ortho LC (conditioned). Fuji Ortho LC (not-conditioned) produced the lowest SBS values with all the bracket base designs tested in this study.

- Of all the cements tested (independent of bracket base design), Transbond and Fuji Ortho LC (conditioned) produced the best fracture mode by leaving more than half of the cement on the tooth.

- Conditioning with $10 \%$ polyacrylic acid had a statistically and clinically significant effect on the SBS and ARI scores. Based on these results, the not-conditioned protocol 
when bonding with Fuji Ortho LC cement was not repeated for the long-term storage study.

- Transbond and Fuji Ortho LC conditioned cements were both compatible with the four bracket bases tested in producing clinically acceptable SBS and ARI scores, however, the most ideal cement for all the bracket base types tested in this study was Fuji Ortho LC (conditioned).

- Panavia21 cement showed that it had limited application in orthodontic bonding. Although it resulted in favorable bond failure patterns when used to bond ceramic brackets, bond failure patterns were unacceptable when Panavia21 was used to bond metal brackets. Panavia2l has chemical adhesive properties to metal but not to nonsilanated ceramic. During debonding of metal brackets bonded with Panavia21, most of the cement was left on the bracket base.

- Of all the variables that can affect SBS and ARI scores, enamel conditioning had the most significant influence. Conditioning with $10 \%$ polyacrylic acid was a requirement when bonding with Fuji Ortho LC cement.

- Long-term storage had no effect on the clinical acceptability of various bracket basecement combinations. After 1 and 180-days of storage, the Speed-Panavia21 and TimePanavia2l combinations produced unacceptable ARI scores. Storage time of 180-days had a significant effect on the SBS of $25 \%$ (3 out of 12) of the groups tested. After 180days of storage, $17 \%$ of the samples showed statistically significant $(P<0.05)$ increase in SBS and $8 \%$ showed a significant reduction in SBS. These changes were not, however, clinically significant.

- Un-weighted Kappa confirmed agreement within and between investigators when assigning ARI scores. There was consistency in assigning ARI scores by the same investigator over time, and also between two different investigators. 
- There was no consistent association between the ARI scores and SBS in this study.

- The particle sizes of Fuji Ortho LC, Transbond, and Panavia21 cements were compatible with base designs of Speed, Time, Transcend, and Clarity brackets. 


\section{BIBLIOGRAPHY}

1. American Association of Orthodontics. Summary of ceramic bracket survey. The Bulletin Supplement. 1989. 7:(Winter)

2. American Dental Association. Council of dental materials, instruments and equipment. Visible light-cured composites and activating units. J Am Dent Assoc. 1985. 110:100103

3. Angolkar PV, Kapila S, Duncanson MG, Nanda RS. Evaluation of friction between ceramic brackets and orthodontic wires of four alloys. Am J Orthod Dentofacial Orthop. 1990. 98:499-506

4. Antonucci JM, McKinney JE, Stansbury JW. Resin-modified glass ionomer cement. US Patent Application No.7-160 856, 1988

5. Armas HR, Sadowsky PL, Vlachos C, Jacobson A, Wallace D. An in vivo comparison between a visible light-cured bonding system and a chemically cured bonding system. Am J Orthod Dentofacial Orthop. 1998. 113:271-5

6. Artun J, Bergland S. Clinical trials with crystal growth conditioning as an alternative to acid-etch enamel pre-treatment. Am J Orthod. 1984. 85(4): 333-340

7. Ashcraft DB, Staley RN, Jakobsen JR. Fluoride release and shear bond strength of three light-cured glass ionomer cements. Am J Orthod Dentofacial Orthop. 1997. $111: 260-265$

8. Bagnall RD. Adhesion in dentistry. Part 1. Critical overview. Mater Sci Technol. 1989. 5: $621-626$ 
9. Barkmeier WW, Gwinnett AJ, Shaffer SE. Effects of reduced acid concentration and etching time on bond strength and enamel morphology. J Clin Orthod. 1987. 21:395398

10. Beech DR. A spectroscopic study of the interaction between human tooth enamel and polyacrylic acid. Arch Oral Biology. 1972. 17:907-911

11. Beress A, Tjan AH, Schlenker WL. Bond strengths of two resin-modified Glass polyalkenoate cements under different surface conditions. J Clin Orthod. 1998. 32(5):299-302

12. Birdsell DC, Bannon PJ, Web RB. Harmful effects of the ultraviolet radiation used for polymerisation of a sealant and a composite resin. J Am Dent Assoc. 1977. 94:31 1-314

13. Bishara S E, Khowassah MA, Oesterle LJ. Effects of humidity and temperature changes on orthodontic direct-bonding adhesive systems. J Dent Res. 1975. 54(4):751758

14. Bishara SE, Trulove TS. Comparisons of different debonding techniques for ceramic brackets: An in vitro study. Part I and II. Am J Orthod Dentofacial Orthop. 1990. 98:145-153, 263-273

15. Bishara SE, Fehr DE, Jakobsen JR. A comparative study of the debonding strength of different ceramic brackets, enamel conditioners and adhesives. Am J Orthod Dentofacial Orthop. 1993.104:170-179

16. Bishara SE, Fehr DE. Ceramic brackets: Something old, something new, A review. Seminars in Orthod. 1997. 3:178-188 
17. Bishara SE, Gordan VV, VonWald L, Olson ME. Effect of an acidic primer on shear bond strength of orthodontic brackets. Am J Orthod Dentofacial Orthop. 1998a. 114:243-247

18. Bishara SE, Olsen ME, Damon P, Jakobsen JR. Evaluation of a new light-cured orthodontic bonding adhesive. Am J Orthod Dentofacial Orthop. 1998b. 114:80-87

19. Bishara ES, Gordan VV, VonWald L, Jakobsen JR. Shear bond strength of composite, glass ionomer, and acidic primer adhesive systems. Am J Orthod Dentofacial Orthop. 1999a. 115:24-28

20. Bishara SE, VonWald L, Oslen ME, Laffoon JF. Effects of time on shear bond strength of glass ionomer and composite bonded orthodontic brackets. Am J Orthod Dentofacial Orthop. 1999b. 116:616-620

21. Bishara SE, VonWald L, Laffoon JF. Effects of altering the type of enamel conditioner on the shear bond strength of a resin-reinforced glass ionomer adhesive. Am J Orthod Dentofacial Orthop. 2000. 118:288-294

22. Black RB. Airbrasive: some fundamentals. J Am Dent Assoc. 1950. 41:701-710

23. Bordeaux JM, Moore RN, Bagby MD. Comparative evaluation of ceramic bracket base designs. Am J Ortho Dentofacial Orthop. 1994. 105:552-560

24. Bourke AM, Walls AW, McCabe JF. Light activated glass polyalkenoate (ionomer) cement: the setting reaction. J Dent. 1992. 20:115-120

25. Bowen RL: U.S. Pat. 3,066,112 (Nov. 27, 1962) 
26. Britton J, McInnes P, Weinberg R, Ledoux W, Retief DH. Shear bond strength of ceramic orthodontic brackets to enamel. Am J Orthod Dentofacial Orthop. 1992. 98:348-353

27. Brown CRL, Way DC. Enamel loss during orthodontic bonding and subsequent loss during removal of filled and unfilled adhesives. Am J Orthod. 1978. 74:663-671

28. Buonocore MG. A simple method of increasing the adhesion of acrylic filling material to enamel surfaces. J Dent Res. 1955. 34:849-853

29. Buonocore MG, Matsui A, Gwinnett A. Penetration of resin dental materials into enamel surfaces for bonding. Arch Oral Biol. 1978. 13:61-70

30. Burkey PS. Enamel conditioning with acid etch and crystal bonding techniques: tensile and shear bond strength comparisons and scanning electron microscopic observations. Master Thesis. University of Iowa, Iowa City. 1985.

31. Buzzitta VA, Hallgren SE, Powers JM. Bond strength of orthodontic direct-bonding cement-bracket systems as studied in vitro. Am J Orthod Dentofacial Orthop. 1982. 81:87-92

32. Cacciafesta V, Subenberger U, Jost-Brinkmann $P$, Miethke R. Shear bond strengths of ceramic brackets bonded with different light-cured glass ionomer cements: an in vitro study. European J Ortho. 1998. 20:177-187

33. Carter RN. Clinical management of ceramic brackets. J Clin Orthod. 1989. 23:807809

34. Chamda R, Stein E. Time-related bond strength of light-cured and chemically cured bonding systems: an in vitro study. Am J Orthod Dentofacial Orthop. 1996.110:378382 
35. Cho E, Kopel H, White SN. Moisture susceptibility of resin-modified glass ionomer materials. Quint Int. 1995. 26(5): 351-358

36. Compton AM, Meyers C, Hondrum S, Lorton L. Comparison of the shear bond strength of a light cured glass ionomer and a chemically cured glass ionomer for use as an orthodontic agent. Am J Orthod Dentofacial Orthop. 1992. 104:138-144

37. Cook PA. Direct bonding with glass ionomer cement. J Clin Orthod. 1990. 8:509-511

38. Cook PA, Youngson CC. An in vitro study of bond strength of glass ionomer cement in the direct bonding of orthodontic brackets. Br J Orthod. 1988.15:247-253

39. Cook WD. Factors affecting the depth of cure of UV polymerized composites. J Dent Res. 1980. 59:800-808

40. Coups Smith KS. The relationship between bond strength and bonding agent when resin-reinforced glass ionomer cements are used to bond oithodontic attachments to bovine enamel. Diploma Thesis. University of Toronto, Ontario, Canada. 1997.

41. Craig RG. Restorative Dental Materials (ed 10). Mosby Publishing. St. Louis. 1997. P.196-198

42. Creanor SL, Carruthers LMC, Saunders WP, Strang R, Foye RH. Fluoride uptake and release characteristics of glass ionomer cement. Caries Res. 1994. 28:322-328

43. Crisp S, Wilson AD. Reactions in glass ionomer polyelectrolyte cements. V. Effects of incorporating tartaric acid in the cement liquid. J Dent Res. 1976. 55:1023-1031

44. Deidrich P, Dickmeiss B. Vergleichende physikalische und rasterelektronenoptisanche untersuchungenn zur adhusion versciedener metallbrackets. Fortschritte der 
keiferorthopaedie. 44:298-310, 1983 In: Millet D, McCabe JF, Gordon PH. The role of sandblasting on the retention of metallic brackets applied with glass ionomer cement. Br J Orthod. 1993. 20:117-122

45. Demke R. Material product data sheet, 1996 In: Meehan MP, Foley TF, Mamandras AH. A comparison of the shear bond strengths of two glass ionomer cements. Am J Orthod Dentofacial Orthop. 1999. 115:125-132

46. De Saeytijd C, Carels CL, Lesaffre E. An evaluation of a light-curing composite for bracket placement. European J Orthod. 1994. 16:541-545

47. Dickinson PT, Powers JM. Evaluation of fourteen direct-bonding orthodontic bases. Am J Orthod. 1980. 78:630-639

48. Dogon IL. Studies demonstrating the need for an intermediary resin of low viscosity for the acid etch technique. In: The acid etch technique. North Central Publishing Company. St Paul, Minn. 1975.

49. Douglas WH, Craig RG, Chen CJ. A new composite restorative based on a hydrophobic matrix. J Dent Res. 1979. 58(10): 1981-1986

50. Douglass JB. Enamel wear caused by ceramic brackets. Am J Orthod Dentofacial Orthop. 1989. 95:96-98

51. Eliades T, Lekka M, Eliades G, Brantley WA. Surface characterization of ceramic brackets: a multi-technique approach. Am J Orthod Dentofacial Orthop. 1994. 105:1018

52. Fajen V, Duncanson M, Nanda R, Currier G, Angolkar $P$. An in-vitro evaluation of bond strength of three glass ionomer cements. Am J Orthod Dentofacial Orthop. 1990. 97:316-322 
53. Farquhar RB. Direct bonding comparing a polyacrylic acid and a phosphoric acid technique. Am J Orthod Dentofacial Orthop. 1986. 90:187-194

54. Faust JB, George GN, Fan PL, Powers JM. Penetration coefficient, tensile strength, and bond strength of thirteen direct bonding orthodontic cements. Am J Orthod. 1978. 73:512-525

55. Ferguson JW, Read MJ, Watts DC. Bond strengths of an integral bracket-base. European J Orthod. 1984. 6:267-276

56. Fitzpatrick DA, Way DC. The effects of wear, acid etching, and bond removal on human enamel. Am J Orthod Dentofacial Orthop. 1977. 72:671-681

57. Fleiss JL, Fischman SL, Chilton NW, Park MH. Reliability of discrete measurements in caries trials. Caries Res. 1979. 13:23-31

58. Flores A, Saez G, Barcelo F. Metallic bracket to enamel bonding with a photopolymerizable resin-reinforced glass ionomer. Am J Orthod Dentofacial Orthop. 1999. 116:514-517

59. Forss H, Seppa L. Prevention of enamel demineralization adjacent to glass ionomer filling materials. Scand J Dent Res. 1990. 98:173-178

60. Fowler CS, Swartz ML, Moore BK, Rhodes BF. Influence of selected variables on adhesion testing. Dent Mater. 1992. 8:265-269

61. Fox NA. Fluoride release from orthodontic bonding materials: $\mathrm{An}$ in vitro study. $\mathrm{Br} \mathrm{J}$ Orthod. 1990. 7:293-298 
62. Fox NA, McCabe JF, Gordon PH. Bond strengths of orthodontic bonding material: An in vitro study. $\mathrm{Br} \mathrm{J}$ Orthod. 1991. 18:125-130

63. Fox NA, McCabe JF, Buckley JG. A critique of bond strength testing in orthodontics. Br J Orthod. 1994. 21:33-43

64. Fox NA, McCabe JF. An easily removable ceramic bracket? Br J Orthod. 1992. 19:305-309

65. Franklin S, Garcia-Godoy F. Shear bond strength and effects on enamel of two ceramic brackets. J Clin Orthod. 1993. 27:83-88

66. Fricker JP. A 12-month clinical evaluation of a light-activated glass polyalkenoate (ionomer) cement for the direct bonding of orthodontic brackets. Am J Orthod. 1994. 105:502-505

67. Fujita T. Histology of teeth. Tokyo: Ishiyaku Shuppan; 1957. P.21-132

68. Goldstein RE, Parkins FM. Air-abrasive technology: its new role in restorative dentistry. J Am Dent Assoc. 1994. 125:551-557

69. Gorelick L, Geiger AM, Gwinnett JA. Incidence of white spot formation after bonding and banding. Am J Orthod. 1982. 81: 93-98

70. Gorelick L, Masunaga G, Thomas RG. Round Table on bonding. J Clin Orthod. 1978; 12:695-714; 761-778; 825-842

71. Greenlaw R, Way DC, Gail KA. An in-vitro evaluation of visible light-cured resin as an alternative to conventional resin bonding systems. Am J Orthod Dentofacial Orthop. 1989. 96:214-220 
72. Guess MB, Watanabe LG, Beck FM, Crall MG. The effect of silane coupling agents on the bond strength of a polycrystalline ceramic bracket. J Clin Orhod. 1988. 22:788-792

73. Gwinnett AJ, Matsui A. A study of enamel adhesives: the physical relationship between enamel and adhesive. Arch Oral Biol. 1967. 12:1615-1620

74. Gwinnett AJ. A comparison of shear bond strengths of metal and ceramic brackets. Am J Orthod Dentofacial Orthop. 1988. 93:346-348

75. Hallgren A, Oliveby A, Twetman S. Caries associated microflora in plaque from orthodontic appliances retained with glass ionomer cement. Scand J Dent Res. 1992. 100:140-143

76. Hanson GH, Gibbon WM, Shimizu H. Bonding bases coated with porous metal powder: a comparison with foil mesh. Am J Orthod. 1983. 83:1-4

77. Harris AMP, Joseph VP, Rossouw PE. Comparison of shear bond strengths of orthodontic resins to ceramic and metal brackets. J Clin Orthod. 1990. 24:725-728

78. Hatibovic-Kofman $\mathrm{S}$, Koch $\mathrm{G}$. Fluoride release from glass ionomer cements in vivo and in vitro. Swed Dent J. 1991. 15:253-258

79. Hotz P, McLean JW, Wilson AD. The bonding of glass ionomer cements to metal and tooth substrates. Br Dent J. 1977. 142:41-47

80. Hyer KE. An in vitro study of shear and tensile bond strengths comparing mechanically and chemically bonded ceramic brackets with three bonding agents. Master Thesis. University of Iowa, Iowa City. 1989.

81. Iwamoto $\mathrm{H}$, Kawamoto $\mathrm{T}$, Kinoshita $\mathrm{Z}$. Bond strength of new ceramic brackets as studied in vitro. J Dent Res. 1987. 66:928 (abstract) 
82. Johnson WT, Hembree JH, Weber FN. Shear bond strengths of orthodontic direct bonding adhesives. Am J Orthod. 1976. 70:559-566

83. Joseph VP, Rossouw PE. The shear bond strengths of stainless steel and ceramic brackets used with chemically and light-activated composite resins. Am J Orthod Dentofacial Orthop. 1990. 97:121-125

84. Karamouzos A, Athanasiou AE, Papadooulos MA. Clinical characteristics and properties of ceramic brackets: A comprehensive review. Am J Orthod Dentofacial Orthop. 1997. 112:34-40

85. Katona TR. Stresses developed during clinical debonding of stainless steel orthodontic brackets. Angle Orthod. 1997. 67(1): 39-46

86. King L, Smith RT, Wendt SL. Bond strengths of lingual orthodontic brackets bonded with light-cured composite resins cured by transillumination. Am J Ortho Dentofacial Orthop. 1987. 91:312-315

87. Kitasako Y, Yamada T, Harada N, Sonoda H. Study on adhesive resin cements. Part I. Several properties of various cements. JPN J Conser Dent. 1994. 37:41 1-422

88. Kittipibul P, Godfrey K. In vitro shearing force testing of the Australian Zirconia-based ceramic Begg bracket. Am J Orthod Dentofacial Orthop. 1995. 108(3):308-315

89. Knibbs PJ, Plant CG, Shovelton DS. The performance of a zinc polycarboxylate luting cement and a glass ionomer luting cement in general dental practice. Br Dent J. 1986. 160:13-15

90. Lalani N, Foley TF, Voth R, Banting D, Mamandras AH. Polymerisation with the argon laser: curing time and shear bond strength. Angle Orthod. 1999. 69:525-534 
91. Legler LR, Retief DH, Bradley EL. The effects of phosphoric acid concentration and etch duration on the shear bond strength of an orthodontic bonding resin to enamel: An in vitro study. Am J Orthod Dentofacial Orthop. 1989. 96:485-492

92. Leichester H. Biochemistry of the teeth. Mosby Publishing. St. Louis. 1949. P.13-102

93. Leung RL, Fan PL, Johnston WM. Post-irradiation of visible light activated composite resin. J Dent Res. 1983. 62:263-265

94. Lippitz SJ, Staley RN, Jakobsen JR. In vitro study of 24-hour and 30-day shear bond strengths of three resin-glass ionomer cements used to bond orthodontic brackets. Am J Orthod Dentofacial Orthop. 1998. 113:620-624

95. Lopez JI. Retention shear strengths of various bonding attachment bases. Am J Orthod. 1980. 77:669-678

96. Low T, von Fraunhofer JA. The direct use of composite materials in adhesive dentistry. Br J Orthod. 1976. 141:207-213

97. MacColl G. The relationship between bond strength and base surface area using conventional and micro-etched foil-mesh bases. Diploma Thesis. Faculty of Dentistry, University of Toronto, Canada. 1995.

98. Machen DE. Legal aspect of orthodontic practice: risk management concepts, ceramic bracket update. Am J Orthod Dentofacial Orthop. 1990. 98:185-186

99. Mahal RS. A standardized approach to determine the effect of thermocycling and longterm storage on the shear bond strength of orthodontic brackets cemented to bovine enamel. Masters Thesis. Faculty of Dentistry, University of Toronto, Canada. 2000. 
100. Maijer R, Smith DC. A new surface treatment for bonding. J Biomed Mater Res. 1979. 13:975-985

101. Maijer R, Smith DC. Variables influencing the bond strength of metal orthodontic bracket bases. Am J Orthod. 1981. 79:20-34

102. Maijer R, Smith DC. Crystal growth on the outer enamel surface: An alternative to acid etching. Am J Orthod. 1986. 89:183-193

103. Maskeroni AJ, Meyers CE, Lorton L. Ceramic bracket bonding: a comparison of bond strength with polyacrylic acid and phosphoric acid enamel conditioning. Am J Orthod Dentofacial Orthop. 1990. 97:168-175

104. Matasa CG. Adhesion and Ten Commandments. Am J Orthod. 1989. 95:355-356

105. McCabe JF, Carrick TE. Output from visible-light activation units and depth of cure of light-activated composites. J Dent Res. 1989. 68:1534-1539

106. McCourt JW, Cooley RL, Barnwell S. Bond strength of light cured fluoride releasing base-liners as orthodontic bracket adhesives. Am J Orthod Dentofacial Orthop. 1991. $100: 47-52$

107. McLean JW, Wilson AD, Prosser HJ. Development after use of water hardening glass ionomer lutting cements. J Prost Dent. 1984. 52:175-181

108. McSherry PF. An in vitro evaluation of the tensile and shear strengths of four adhesives used in orthodontics. European J Orthod. 1996. 18:319-327

109. Meehan MP, Foley TF, Mamandras AH. A comparison of the shear bond strengths of two glass ionomer cements. Am J Orthod Dento Orthop. 1999. 115:125-132 
110. Meng C, Wang W, Trang T, Lue Y, Lai J, Arvyatas MG. Orthodontic resin under water immersion. Angle Orthod. 1995. 65(3):209-214

111. Merrill S, Oesterle LJ, Hermesch CB. Ceramic bracket bonding: A comparison of shear, tensile, and torsional bond strengths of ceramic brackets. Am J Orthod Dentofacial Orthop. 1994. 106:290-297

112. Miller JR, Mancl L, Arbuckle G, Baldwin J, Phillips RW. A three-year clinical trial using glass ionomer cement for the bonding of orthodontic brackets. Angle Orthod. 1996. 66:309-312

113. Millett D, McCabe JF, Gordon PH. The role of sandblasting on the retention of metallic brackets applied with glass ionomer cements. Br J Orthod. 1993. 20(2):117122

114. Millett DT, McCabe JF. Orthodontic bonding with glass ionomer cement: a review. European J Orthod. 1996. 18:385-399.

115. Millett DT, Cattanach D, McFadzean R, Pattison J, McColl J. Laboratory evaluation of a compomer and a resin-modified glass ionomer cement for orthodontic bonding. Angle Orthod. 1999. 69(1):58-64

1 16. Mitchell L. Decalcification during orthodontic treatment with fixed appliances - an overview. Br J Orthod. 1992. 19:199-205

117. Moriwaki Y, Kani T, Kozatani T, Tsutsumi S, Shimode N, Yamaga R. The crystallinity change of bovine enamel during maturation. JPN J Dent Mater. 1968. 9:78-85

118. Miura F, Nakagawa K, Masuhara E. New direct bonding system for plastic brackets. Am J Orthod. 1971. 59:350-359 
119. Nakamichi I, Iwaku M, Fusayama T. Bovine teeth as possible substitute in adhesion tests. J Dent Res. 1983. 62:1076-1081

120. Newman GV. Epoxy adhesives for orthodontic attachments: Progress report. Am J Orthod. 1965. 101:190-191

121. Newman GV, Snyder WH, Wilson CE. Acrylic adhesives for bonding attachments to tooth surfaces. Angle Orthod. 1968. 38:12-18

122. Newman GV, Murray GA, Yates JL. Visible lights and visible light-activated composite resins. J Prost Dent. 1983. 50(1):31-35

123. Newman GV, Newman RA, Sun BI, Ha JJ, Ozsoylu SA. Sandblasting, Silanating, and coating: Their effects on bond strength of metal brackets: An in vitro study. J New Jersey Dent Assoc. 1995. 15-23

124. Norevall LI, Marcusson A, Persson M. A clinical evaluation of glass ionomer cement. European J Orthod. 1995. 17:449 (Abstract)

125. Norevall LI, Marcusson A, Persson M. A clinical evaluation of a glass ionomer cement as an orthodontic bonding adhesive compared with an acrylic resin. European $J$ Orthod. 1996. 18:373-384

126. Nouri MR. Glass Ionomer Compounds: chemistry, biochemistry, adhesion, fluoride release, and mechanical properties. Diploma Thesis. Faculty of Dentistry, University of Toronto, Canada. 1997.

127. O'Brien KD, Watts DC, Read MJ. Residual debris and bond strength- is there a relationship? Am J Orthod Dentofacial Orthop. 1988. 94:222-230 
128. O'Brien KD, Read MJ, Sandison RJ, Roberts CT. A visible light activated direct bonding material: an in vivo comparative study. Am J Orthod Dentofacial Orthop. 1989. 95:348-351

129. O'Brien KD, Watts DC, Read MJ. Light-cured direct bonding -is it necessary to use a primer? European J of Orthod. 1991. 13:22-26

130. Odegaard J, Segner $D$. Shear bond strength of metal brackets compared with a new ceramic bracket. Am J Orthod Dentofacial Orthop. 1988. 94:201-206

131. Odegaard J. Debonding ceramic brackets. J Clin Orthod. 1989. 23:632-635

132. Oesterle LJ, Shellhart WC, Belanger G. The use of bovine enamel in bonding studies. Am J Orthod Dentofacial Orthop. 1998. 113:514-519

133. Ogaard B, Rolla G, Arends J. Orthodontic appliances and enamel demineralisation. Part 1. Lesion development. Am J Orthod Dentofacial Orthop. 1988. 94:68-73

134. Olsen ME, Bishara SE, Damon P, Jakobsen JR. Evaluation of Scotch brand multipurpose and maleic acid as altemative methods of bonding orthodontic brackets. Am J Orthod Dentofacial Orthop. 1997. $111: 498-501$

135. Olsen ME, Bishara SE, Boyer D. Effect of varying etching time on the bond strength of ceramic brackets. J Dent Res. 1994. 73:197 (abstract \#766)

136. O'Reilly MM, Featherstone JD. Demineralisation and mineralisation around orthodontic appliances: An in vivo study. Am J Orthod Dentofacial Orthop. 1987. 92:33-40 
137. Ostertag AJ, Dhuru VB, Ferguson DJ, Meyer RA Jr. Shear, torsional, and tensile bond strengths of ceramic brackets using three adhesive filler concentrations. Am J Orthod Dentofacial Orthop. 1991. 100:251-258

138. Ostertag AJ, Dhuru VB, Ferguson DJ. In-vitro tensile bond strength comparison of metal and ceramic brackets using auto-polymerising and light-cured resins. Am J Orthod Dentofac Orthop. 1989. 97:531 (abstract)

139. Ostman-Andersson E, Marcusson A, Horstedt P. Comparative SEM studies of the enamel surface after debonding following the use of glass ionomer cement and acrylic resins for bracket bonding. Swed Dent J. 1993. 17:139-146

140. Phillips HW. The advent of ceramics. J Clin Orthod. 1988. 22:69-70

141. Phillips RW. Science of Dental Materials (ed 8). Philadelphia, PA: Saunders, 1982. P.45

142. Powers JM, Turner DS, Nakano A. Bonding of hybrid ionomers to unetched enamel and orthodontic brackets. J Dent Res. 1996. 75:173 (abstract \#1247)

143. Powers JM, Kim H, Turner D. Orthodontic adhesive and bond strength. Seminars in Ortho. 1997. 3 (3): 147-156

144. Prattern DH, Popli K, Germane N, Gunsolley J. Frictional resistance of ceramic and stainless steel orthodontic brackets. Am J Orthod Dentofacial Orthop. 1990. 98:398403

145. Proffit WR, and Field HW. Contemporary orthodontics. Mosby Publishing. St. Louis. 1986. P.228-246 
146. Profitt WR, and Field HW, Nixon WL. Occlusal forces in normal and long faced adults. J Dent Res. 1983. 62:566-570

147. Prosser HJ, Powis DR, Wilson AD. Characterization of glass-ionomer cements. 7: The physical properties of current materials. J Dent. 1984. 12: 231-240

148. Pus MD, Way DC. Enamel loss due to orthodontic bonding with filled and unfilled resin using various clean-up techniques. Am J Orthod. 1980. 77:269-283

149. Regan D, van Noort R. Bond strengths of two integral bracket-base combinations: an in vitro comparison with foil-mesh. European J of Orthod. 1989. 11:144-153

150. Retief DH. A comparative study of three etching solutions. Effects on contact angle, rate of etching and tensile bond strength. J Oral Rehab. 1974. 1:381-390

151. Retief DH. The mechanical bond. Int Dent J. 1978. 28:18-27

152. Reynolds IR. A review of direct orthodontic bonding. $\mathrm{Br}$ J Orthod. 1975. 2:171-178

153. Reynolds IR, von Fraunhofer JA. Direct bonding of orthodontic attachments to teeth: the relation of adhesive bond strength to gauze mesh size. Br J Orthod. 1976. 3:91-95

154. Rezk-Lega F, Ogaard B, Arends J. An in vitro study on the merits of two glass ionomers for the cementation of orthodontic bands. Am J Orthod Dentofacial Orthop. 1991. 99:162-167

155. Ripley KT. In vitro comparative study of shear and tensile bond strengths of stainless steel and ceramic orthodontic brackets. Masters Thesis. University of Iowa, Iowa City. 1988. 
156. Rix D, Foley TF, Mamandras A. Comparison of bond strength of three adhesives: Composite resin, hybrid GIC, and glass-filled GIC. Am J Orthod Dentofacial Orthop. 2001. 119:36-42

157. Rock WB. The use of ultra-violet radiation in dentistry. Br Dent J. 1974. 136:455-60

158. Rueggeberg FA. Substrate for adhesion testing to tooth structure-review of the literature. Dental Mater. 1991. 7:2-10

159. Rueggeberg FA, Caughman WF, Curtis JW Jr. Effect of light intensity and exposure duration on cure of resin composite. Oper Dent. 1994. 19:26-32

160. Rux W, Cooley RL, Hicks JL. Evaluation of a phosphonate BIS-GMA resin as a bracket adhesive. Quintess Int. 1991. 22:57-60

161. Ruyter JE, Oysaed H. Conversion in different depths of ultraviolet and visible light activated composite materials. Acta Odontol Scand. 1982. 40(3):179-192

162. Sadowsky PL, Retief DH, Cox PR. Effects of etchant concentration and duration on the retention of orthodontic brackets: An in vivo study. Am J Orthod Dentofacial Orthop. 1990. 98:417-421

163. Salako NO, Cruickshanks-Boyd DW. Curing depths of materials polymerised by ultra-violet light. Br Dent J. 1979. 146:375-381

164. Sam WH, Chao SY, Chung KH. Shear bond strength of ceramic brackets with two bonding adhesives. Br J Orthod. 1993. 20:225-229

165. Sargison A, McCabe JF, Gordon P. An Ex Vivo study of self-, light-, and dual-cured composites for orthodontic bonding. Br J Orthod. 1995. 22:319-323 
166. Sharma SK. The influence of bracket base design on shear bond strength of brackets bonded with bovine enamel. Diploma Thesis. Faculty of Dentistry, University of Toronto, Canada. 1999.

167. Shen C, Grimaudo N. Effect of hydration on the biaxial flexural strength of glass ionomer cement. Dent Mater. 1994. 10:190-195

168. Sidhu SK, Watson TF. Resin modified glass ionomer materials. A status report for the American Journal of Dentistry. Am J Dent. 1995. 8:59-67

169. Silverman E, Cohen M, Demke R, Silverman M. A new light cured glass ionomer cement that bonds brackets to teeth without etching in the presence of saliva. Am J Orthod. 1995. 108:123-126

170. Silverstone LM, Saxton CA, Dogon IL, Fejerskov O. Variations in the pattern of acid etching of human dental enamel examined by scanning electron microscopy. Caries Res. 1975. 9:373-387

171. Simoka LV, Powers JM. In Vitro bond strength of treated direct-bonding metal bases. Am J Orthod. 1985. 88:133-136

172. Sinha PK, Rohrer MD, Nanda RS, Brickman CD. Interlayer formation and its effects on debonding polycrystalline ceramic orthodontic brackets. Am J Orthod Dentofacial Orthop. 1995. 108:455-463

173. Smith DC. A new dental cement. Br Dent J. 1968; 125:381-394

174. Smith DC, Cartz L. Crystalline interface formed by polyacrylic acid and tooth enamel. J Dent Res. 1973; 52:1155 
175. Smith H, Casko J, Leinfelder K, Utley JD. Comparison of orthodontic bracket bond strengths: human vs. bovine enamel. J Dent Res. 1976. 55:153-167

176. Smith DC, Williams DF. Biocompatibility of dental materials. Volume II, Boca Raton, Fla: CRC Press Inc. 1982. P.51-76

177. Smith DC, Maijer R. Improvements in bracket base design. Am J Orthod. 1983. 83(4):277-281

178. Storm ER. Debonding ceramic brackets. J Clin Orthod. 1990. 24:91-94

179. Strobl K, Bahna TL, Wilham L. Laser-aided debonding of orthodontic ceramic brackets. Am J Orthod Dentofacial Orthop. 1992. 152-159

180. Suga $\mathrm{S}$, Kondo $\mathrm{M}$, Onodera $\mathrm{K}$, Kubora $\mathrm{Y}$, Ohtsuka $\mathrm{M}$. Electron microscope analysis of the distributions of $\mathrm{Cl}, \mathrm{Mg}$, and $\mathrm{Na}$ in the enamels of various animals. JPN J Oral Biol. 1971. 13:85-94

181. Swartz ML. Ceramic brackets. J Clin Orthod. 1988a. 22:82-88

182. Swartz ML, Ormco Corporation. A technical bulletin on the issue of bonding and debonding ceramic brackets. 1988b. \#070-5039

183. Swift EJ. Glass ionomer cements. A review. Miss Dent Assoc J. 1986. 42(1):10-12

184. Swift EJ, Cloe BC. Shear bond strengths of new enamel etchants. Am J Dent. 1993. 6(3):162-164

185. Tang ATH, Bjorkman L, Isaksson L. Retrospective study of orthodontic bonding without liquid resin. Am J Orthod Dentofacial Orthop. 2000. 118:300-306 
186. Tavas MA, Watts DC. Bonding orthodontic brackets by transillumination of a light activated composite: an in vitro study. $\mathrm{Br} \mathrm{J}$ Orthod. 1979. 6:207-208

187. Tavas MA, Watts DC. A visible light activated direct bonding material: an in vitro study. Br J Orthod. 1984. 11:33-37

188. Todo A, Tosaki S, Hirota $\mathrm{K}$. The bonding strength of light-cured glass-ionomer cement for orthodontics. Presented at the $3^{\text {rd }}$ international congress on dental materials. 1997.

189. Thanos CE, Munholland T, Caputo A. Adhesion of mesh-based direct bonding brackets. Am J Orthod. 1979. 75:421-430

190. Thompson RE, Way DC. Enamel loss due to prophylaxis and multiple bonding/debonding of orthodontic attachments. Am J Orthod. 1981. 79:282-295

191. Triolo PT, Swift EJ, Mudgil A, Levine A. Effects of etching time on enamel bond strengths. Am J Dent. 1993. 6:302-304

192. Urabe $H$. The effect of different acid conditioning agents on metal, ceramic, and polycarbonate brackets bonded with bovine enamel. Diploma Thesis. Faculty of Dentistry, University of Toronto, Canada. 1996.

193. van Noort R. Introduction to Dental Materials. Mosby Publishing. St. Louis. 1994. P.120-121

194. Viazis AD, Cavanaugh G, Bevis RR. Bond strength of ceramic brackets under shear stress: an in vitro report. Am J Orthod Dentofacial Orthop. 1990. 98:214-221

195. Voss A, Hickel R, Molkner S. In vivo bonding of orthodontic brackets with glass ionomer cement. Angle Orthod. 1993. 2:149-153 
196. Wang JD, Hume WR. Diffusion of hydrogen ion and hydroxyl ion from various sources through dentin. Int Endo J. 1988. 21:17-21

197. Wang WN, Lu TC. Bond strength with various etching times on young permanent teeth. Am J Orthod Dentofacial Orthop. 1991. 100:72-79

198. Wang WN, Meng CL. A study of bond strength between light and self-cured orthodontic resin. Am J Orthod Dentofacial Orthop. 1992. 101:350-354

199. Wang WN, Tarng TH. Evaluation of the sealant in orthodontic bonding. Am J Orthod Dentofacial Orthop. 1991. 100:209-211

200. Watts DC, Amer O, Combe EC. Characteristics of visible-light activated composite systems. Br Dent J. 1984. 156:209-215

201. Waveren Hogervorst WL, Felizer AJ, Prahl-Andersen B. The air-abrasion technique versus the conventional acid-etching technique: a qualification of surface enamel loss and a comparison of shear bond strength. Am J Orthod Dentofacial Orthop. 2000. 117:20-26

202. Weisser JI. Successful method for bonding stainless steel brackets and auxiliaries. J Clin Orthod. 1973. 7: 637-645

203. White LW. Glass ionomer cement. J Clin Orthod. 1986. 20:387-391

204. Wickwire NA, Rentz D. Enamel pretreatment: a critical variable in direct bonding systems. Am J Orthod. 1973. 64:499-412

205. Willems $G$, Carels $C E$, Veerbeke $G$. In vitro peel/shear bond strength evaluation of orthodontic bracket base design. J Dent. 1997. 25:271-278 
206. Wilson $\mathrm{AD}$. Resin modified glass ionomer cements. Int J Prost. 1990. 3:452-429

207. Wilson AD, Groffman DM, Kuhn AT. Fluoride release from fluoride-containing liners/bases. Quint Int. 1990. 21:41-45

208. Wilson AD, Kent BE. A New translucent cement for dentistry. Br Dent J. 1972. 132:133-135

209. Wilson AD, McLean JW. Glass ionomer cement. Chicago, Quintessence Publishing Co. 1988

210. Wiltshire $W$. Shear bond strengths of a glass ionomer for direct bonding in orthodontics. Am J Orthod Dentofacial Orthop. 1994. 106:127-130

211. Wood DP, Jordan RE, Way DC, Galil KA. Bonding to porcelain and gold. Am J Orthod. 1982. 89:194-205

212. Wright AB, Lee RT, Lynch E, Young KA. Clinical and microbiological evaluation of a resin modified glass ionomer cement for orthodontic bonding. Am J Orthod Dentofacial Orthop. 1996. 110:469-475

213. Yamaguchi R, Powers JM, Dennison JB. Parameters affecting in vitro bond strength of composites to enamel and dentine. Dent Mater. 1989. 5(3):153-156

214. Yu KC, Chang R. Adhesive restorative dental materials II. Approaches to adhesive adhesion, national institute of dental research. US dept of health Ed and Welfare. 1966. 103-131 
215. Zachrisson BU. Oral Hygiene for orthodontic patients: current concepts and practical advice. Am J Orthod. 1974. 66:487-497

216. Zachrisson BU. Cause and prevention of injuries to teeth and supporting structures during orthodontic treatment. Am J Orthod. 1976. 69:285-300

217. Zachrisson BU. A post-treatment evaluation of direct bonding in orthodontics. Am J Orthod. 1977. 71:173-189

218. Zachrisson BU. Bonding in orthodontics. In Graber, T.M. Vanarsdall RL. Orthodontics-Current Principles and Techniques. Mosby Publishing. St. Louis. 1994. P.555-559 